U.S. Department of the Interior

U.S. Geological Survey

\title{
Maps and Database of Quaternary Faults in Bolivia and Chile
}

By
Alain Lavenu, Ricardo Thiele, Michael N. Machette,
Richard L. Dart, Lee-Ann Bradley and Kathleen M.
Haller

\section{Open-File Report 00-283}

This report is preliminary and has not been reviewed for conformity with U.S. Geological Survey editorial standards nor with the North American Stratigraphic Code. Any use of trade names in this publication is for descriptive purposes only and does not imply endorsement by the U.S. Geological Survey.

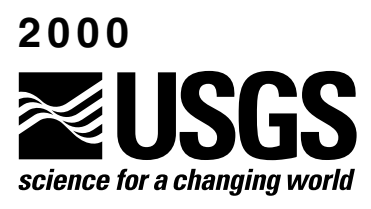




\title{
Map and Database of Quaternary Faults in Bolivia and Chile
}

\section{A project of the International Lithosphere Program Task Group II-2, Major Active Faults of the World \\ Proyecto Internacional de la Litósfera, Grupo de Trabajo II-2, Principales Fallas Activas del Mundo}

\author{
Data and map compiled by \\ Datos y mapa compilado por \\ ${ }^{1}$ ALAIN LAVENU, ${ }^{2}$ RICARDO THIELE, ${ }^{3}$ MICHAEL N. MACHETTE, \\ ${ }^{3}$ RICHARD L. DART, ${ }^{3}$ LEE-ANN BRADLEY, AND ${ }^{3}$ KATHLEEN M. HALLER \\ ${ }^{1}$ IRD (Institut de Recherche pour le Développement) \\ 213 rue La Fayette \\ 75480 Paris, cedex 10, France \\ ${ }^{2}$ Departamento de Geología \\ Universidad de Chile \\ Casilla 13518, Correo 21 \\ Santiago, Chile \\ ${ }^{3}$ U.S. Geological Survey \\ Central Geologic Hazards Team \\ MS 966, P.O. Box 25046 \\ Denver, Colorado, USA
}

\footnotetext{
Regional Coordinator for South America

Coordinador Regionale por Sur America

CARLOS COSTA

Universidad Nacional de San Luis

Departamento de Geología

Casilla de Correo 320

San Luis, Argentina
}

ILP Task Group II-2 Co-Chairman, Western Hemisphere

ILP Grupo de Trabajo II-2 Co-Chairman, Hemisferio Occidental

MICHAEL MACHETTE

U.S. Geological Survey

Central Geologic Hazards Team

MS 966, P.O. Box 25046

Denver, Colorado, USA

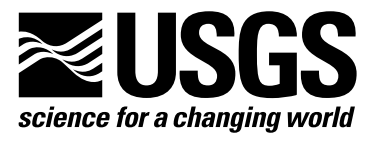

May 2000 Version

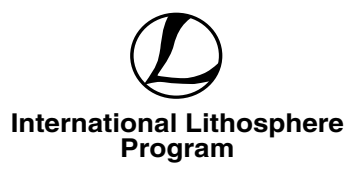




\section{TABLE OF CONTENTS}

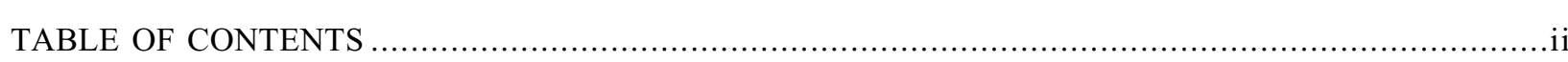

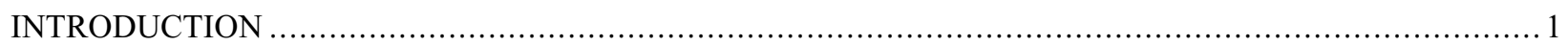

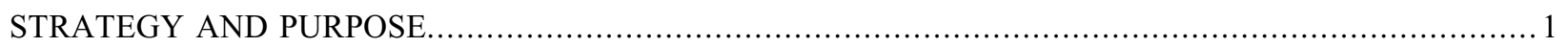

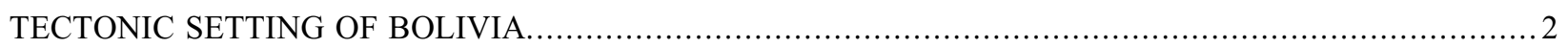

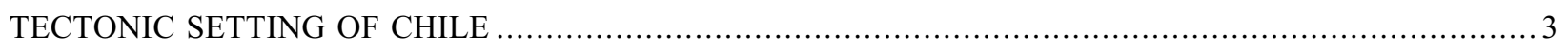

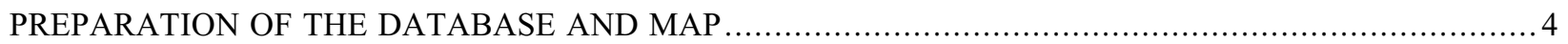

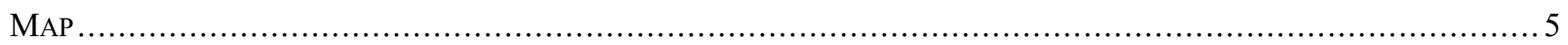

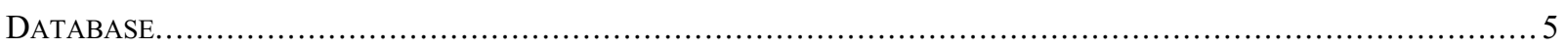

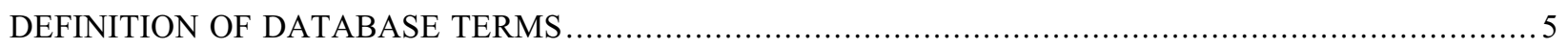

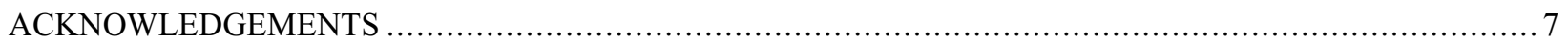

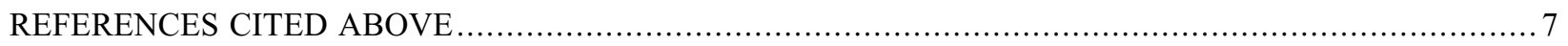

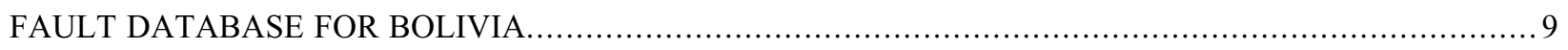

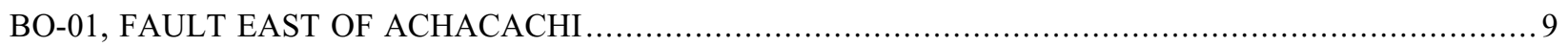

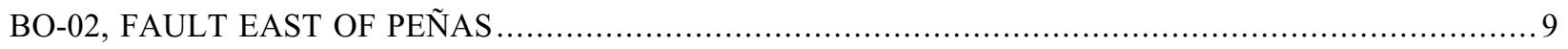

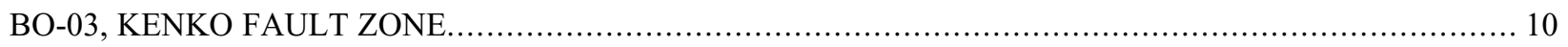

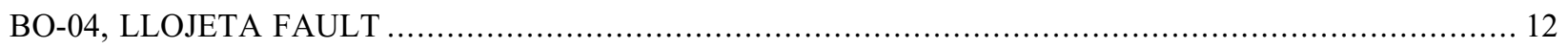

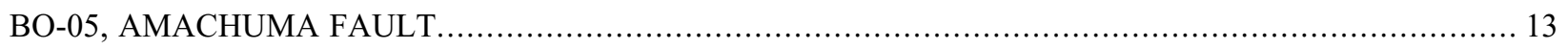

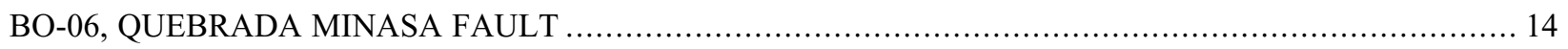

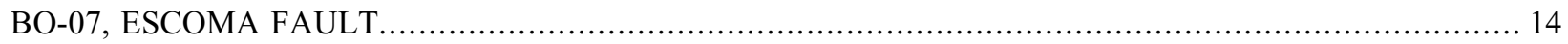

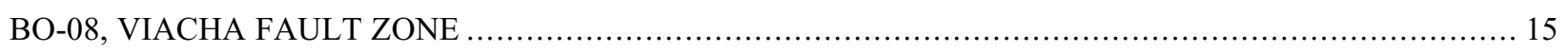

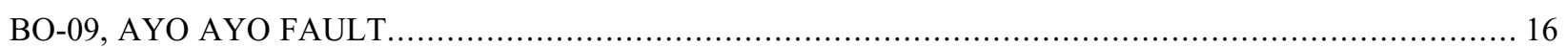

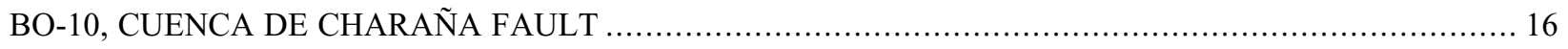

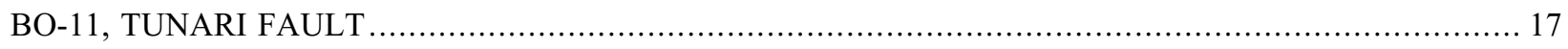

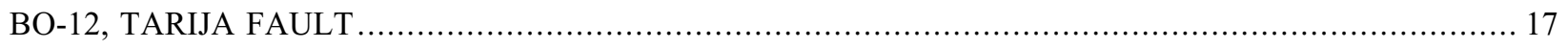

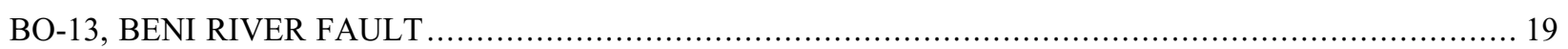

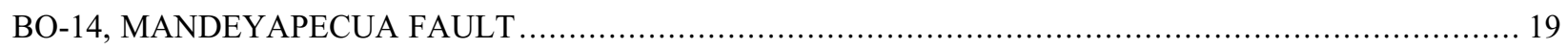

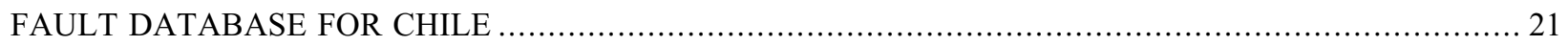

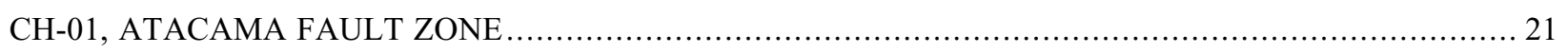

CH-01A, SALAR GRANDE FaUlt, SECTION OF ATACAMA FAULT ................................................. 22

CH-01B, PUNTA DE LOBOS FAUlT, SECTION OF ATACAMA FAULT .................................................. 22

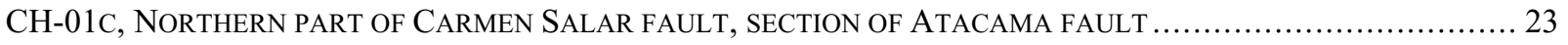

CH-01D, SOUTHERN PART OF CARMEN SALAR FAULT, SECTION OF ATACAMA FAULT................................... 24

CH-01E, Sierra de Remiendos faUlt, SECtion of Atacama faUlt......................................... 25

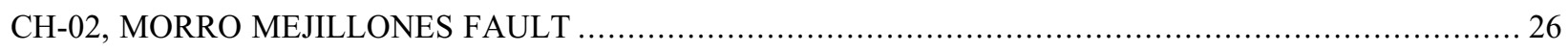

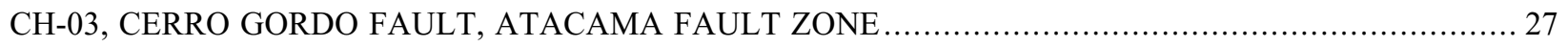

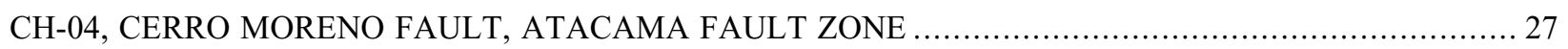

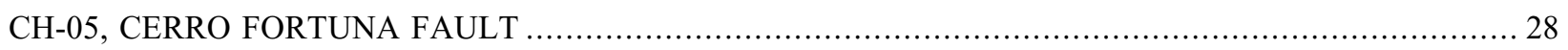

CH-06, WESTERN BACK THRUST FAULT OF CERRO DE PURILACTIS .................................... 29

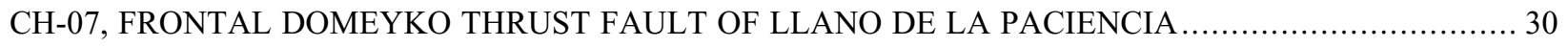

CH-08, IGNIMBRITE BACK THRUST FAULT OF LLANO DE LA PACIENCIA ….............................. 30

CH-09, EASTERN FRONTAL THRUST FAULT OF LLANO DE LA PACIENCIA .................................. 31

CH-10, FRONTAL THRUST FAULT OF CORDILLERA DE LA SAL .............................................. 32

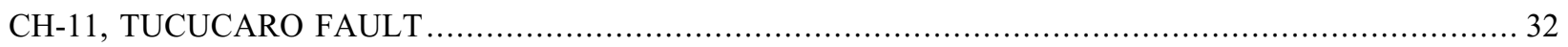




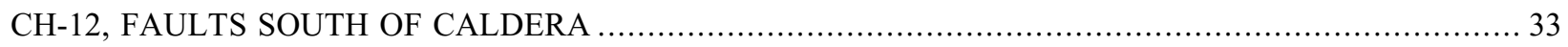

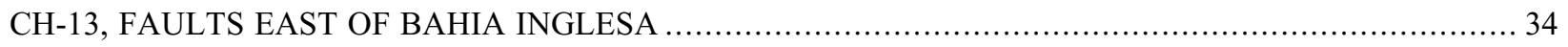

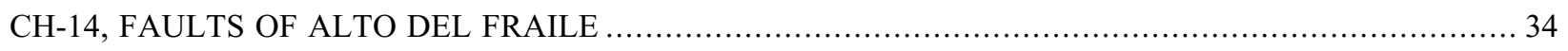

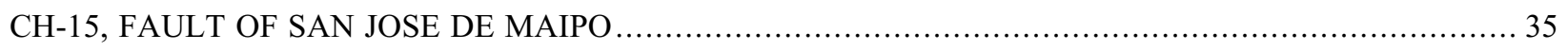

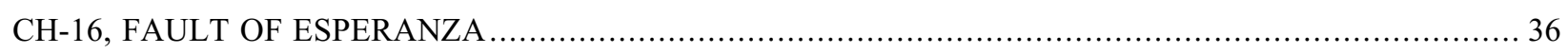

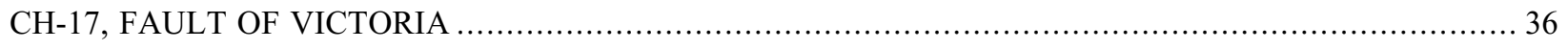

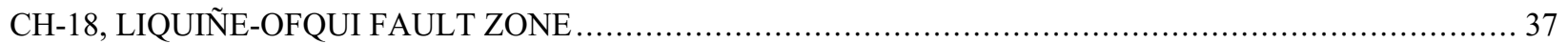

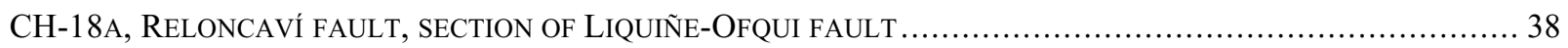

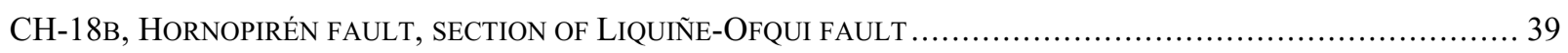

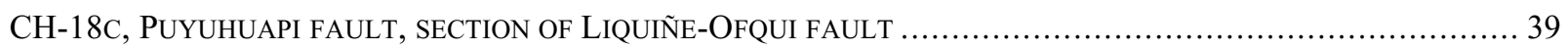

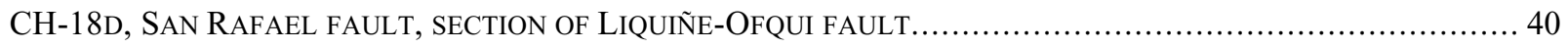

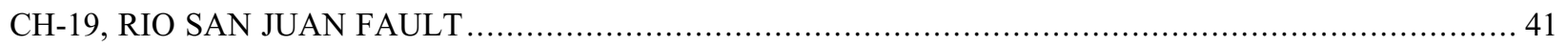

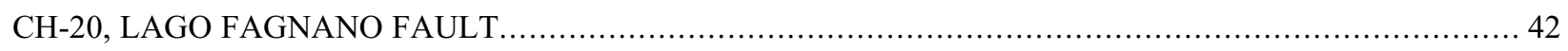

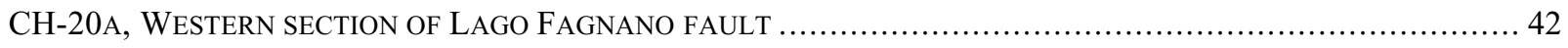

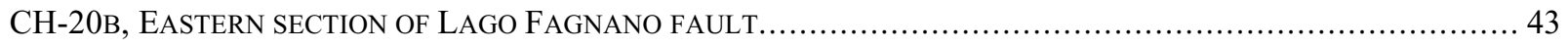

\section{TABLES}

Page

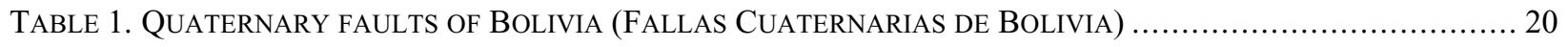

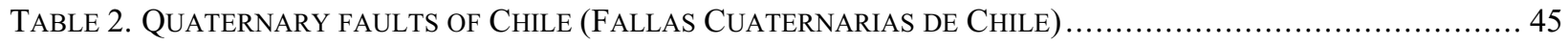




\section{INTRODUCTION}

As part of the International Lithosphere Program's "World Map of Major Active Faults," the U.S. Geological Survey (USGS) is assisting in the compilation of a series of digital maps of Quaternary faults and folds in Western Hemisphere countries. The maps show the locations, ages, and activity rates of major earthquake-related features, such as faults and fault-related folds. They are accompanied by databases that describe these features and document current information on their activity in the Quaternary. The project is a contribution to the International Decade for Natural Hazard Disaster Reduction.

The project is sponsored by the International Lithosphere Program and funded by the USGS's National Earthquake Hazards Reduction Program. The primary elements of the project are general supervision and interpretation of geologic/tectonic information (Michael N. Machette, Project Chief), data compilation and entry for fault catalog (all personnel), database design and management (Kathleen M. Haller), and digitization and manipulation of fault data (Richard L. Dart) in †ARCINFO. For the compilation of data, we engaged experts in Quaternary faulting, neotectonics, paleoseismology, and seismology. These experts are the primary authors of this report, and questions about individual faults should be directed to them. Questions about the project, its status, and the GIS map should be directed to the USGS authors.

Prior to initiating this project, no modern or digital map of active or Quaternary faults existed for Bolivia and Chile or any other country within South America, even though understanding the extent and character of active and older Quaternary faults are critical elements of seismic-hazards analysis. Creation of this map and the accompanying database of 34 faults and faults zones will help extend the relatively short record of instrumental and felt seismicity in Bolivia and Chile by creating a paleoseismic record of surface deformation associated with large (typically $M>6.5$ ) earthquakes.

Although some basic fault data are available for most of Bolivia and Chile, the degree of completeness varies greatly and often is a function of the degree of remoteness and vegetation cover. A few faults have been the subject of recent or more detailed investigations, but none appear to have been trenched for paleoseismologic data. Other regions and faults have been studied in some detail, usually in association with concerns about hazards to urban areas or the safety of critical facilities such as transportation routes, oil-and-gas pipelines, or power-generating facilities. Thus, considerable effort was required from the primary authors in order to compile information from a wide variety of sources and insure that the national product is up to date and provides fairly uniform coverage for the entire country. Nevertheless, the general state of knowledge for faulting in Bolivia and Chile is probably best described as being of a reconnaissance nature. Little is known in a collective sense about the overall rates of fault activity and fault chronology-information that is difficult to acquire but critical to seismic hazard assessments. Hopefully, additional paleoseismic studies will help augment this map and database.

\section{STRATEGY AND PURPOSE}

For the maps of Bolivia and Chile, we relied on Alain Lavenu and Ricardo Thiele, well-informed experts with strong local or regional knowledge of both Bolivia and Chile who were gratious to participate in this international project. Given the limited time to produce the map, the project was restricted to compilation of just those elements needed for ILP's Global Seismic Hazards Assessment Program (see database). No field studies were conducted specifically for this project. However, we anticipate that the project will point out the shortcomings of past and current research on Quaternary faulting in Bolivia and Chile in terms of quantity, quality, scope, and regional coverage and should help promote new efforts to collect paleoseismological data in previously neglected or critical areas.

Many times, seismicity is used to define some potentially active faults, especially along active plate margins. However, several recent faulting events in the Western Hemisphere have shown that much of the faulting away from active plate margins occurs along faults with no significant level of seismicity and that only a fraction of active faults are characterized by ongoing seismicity. Thus, the information on Quaternary faulting included within this database should help extend the modern (past several hundred years) record

\footnotetext{
${ }^{\dagger}$ Any use of trade names (such as this and others in the report) does not imply endorsement by the U.S. Geological Survey.
} 
of seismicity into prehistoric time, and allow better assessments of active and potentially active faults in Bolivia and Chile and other Western Hemisphere countries.

\section{TECTONIC SETTING OF BOLIVIA}

The Bolivian Andes are located above a $30^{\circ}$ east-dipping portion of the subducting Nazca plate. The main characteristics of the Bolivian Andes are 1) the presence of a wide and very subsident (6 to $15 \mathrm{~km}$ deep; Meyer and Murillo, 1961; Martinez, 1980; Lavenu and Marocco, 1984) Tertiary sedimentary basin that forms a high plateau, the Altiplano, at an average elevation of 3,800 meters; 2) the elbow shape of the mountain range, which is referred to as the Bolivian Orocline; 3 ) the Cenozoic and Quaternary volcanic arc, which is still active; 4) a thick crust (55-75 km); and 5) a high amount of topographic relief within and across the range. The high Central Andes of Bolivia, 300 to $600 \mathrm{~km}$ wide, are the wider part of the Andean chain and consist of four morpho-tectonic zones: the Western Cordillera, the Altiplano, the Eastern Cordillera, and the sub-Andean zone.

- The Western Cordillera, which has summits over 6,000 $\mathrm{m}$ in elevation, is in part due to the regional uplift, but essentially results from big volcanoes in the cordillera. The base of these volcanoes is nearly $3,800-4,000 \mathrm{~m}$ in elevation. During the Oligocene and Miocene, this region was a subsident basin (1500 $\mathrm{m}$ deep; Lavenu, 1986) coeval with a calc-alkaline volcanism.

- The Altiplano is between the Western and the Eastern Cordilleras. On this high flat plateau, some features, such as the Serranias, can reach $4,500 \mathrm{~m}$ in elevation. The Altiplano is presently a wide endoreic basin occupied by a lacustrine system (Lakes Titicaca and Poopo) in the northern part, and by salt lakes ("salares") in the central and southern part (e.g., Coipasa and Uyuni Salares). The Western Altiplano, which forms the eastern piedmont of the Western Cordillera, is occuped by Cenozoic volcanic and detritalvolcanic deposits as much as 2,000 m thick. The Central Altiplano, the more subsident Cenozoic zone, is characterized by important continental sedimentation. Faults and folds, resulting from Neogene and Quaternary compressive Andean tectonics, affect this entire region. The Eastern Altiplano, which is the western piedmont of the Eastern Cordillera, is bounded on the east by the important fault-scarp relief of this Cordillera. Only Pliocene deposits outcrop here and the older deposits are masked by widespread Quaternary deposits. During Pliocene time, this region was less deformed than the Central Altiplano, but during Quaternary time the piedmont was affected by collapse along normal faults with significant throw (meter to tens of meters).

- The Eastern Cordillera, comprised essentially of Paleozoic deposits, has been a positive (uplifted) region since the Early Cretaceous. This region, intensely deformed during the Hercynian orogeny, was also affected by Neogene and Quaternary deformation (e.g., Cochabamba and Tarija basins).

- The sub-Andean zone is the eastern piedmont of the Andean Chain (Eastern Cordillera) and consists of a fold-thrust belt. Deformation started here during Oligocene time and continued throughout the Neogene (Baby and others, 1989, 1992) and Quaternary (Dumont, 1996) times. In the foreland, late Pliocene to Quaternary sedimentary units overlie a Pliocene unit with an erosional unconformity (Padula, 1959). A volcanic tuff is dated at $3.3 \pm 0.4 \mathrm{Ma}$ (Moretti and others, 199 ) from the Emborozu Formation, in the lower part of this Plio-Quaternary section. At the front of the Subandean front, the Nuapua Formation, biostratigraphically dated at $0.8 \mathrm{Ma}$ (McFadden and Wolff, 1981) to $0.73 \mathrm{Ma}$ (Marshall and Sempéré, 1991) could correspond to the upper part of the Emborozu Formation. The Ñuapua Formation could correspond also to the "intensely deformed and thrusted deposits" (Baby, unpublished report) outcropping along the Mandeyapecua fault [BO-14], which could confirm recent deformation along the orogenic front.

- The Beni basin is located NE and $\mathrm{E}$ of the Bolivian Andean segment, in the front of the Subandean thrust and fold belt of the Bolivian Andes: The Bolivian Beni basin, as well the Peruvian Marañon basin, is a flexural basin that is subsiding at present. This elongated basin, as well the Madre de Díos Basin, collects the drainage from the eastern slopes of the Andes. The western boundary of the Beni basin with the Andean foothills is a "sharp piedmont line" according to Dumont (1996). Morphological and neotectonic data suggest that the Beni River is forced to the north by a north-striking, more than $65 \mathrm{~km}$ long, fault between the Pando block and the Beni River floodplain. Dumont (1996) observed two N-striking faults [BO-13] cutting the conglomerate and sand layers of an upper terrace of the Beni River. The upthrown side of these faults is on the west. 
During the uppermost Pliocene and after a period of E-W trending extension and syntectonic sedimentation, the Bolivian Andean Chain underwent E-W compressional deformation. During the early Quaternary, a short-lived N-S compressional event affected the entire chain. According to Mercier and others (1992), this deformation could correspond to a rotation of the $\sigma 1$ and the $\sigma 2$ principal axes during late Pliocene time. During the Quaternary, the entire mountain chain was affected by N-S extensional tectonics that can be related to the effect of high topography (Sébrier and others, 1985). This deformation is responsible for important normal faults, the cumulative throw of which reaches more than $400 \mathrm{~m}$ near the town of La Paz. The tectonic basin occupied by Lake Titicaca is normal fault controlled [BO-1, BO-2, BO7], and is one of the consequences of this N-S Quaternary deformation (Lavenu, 1992).

\section{TECTONIC SETTING OF CHILE}

West and mainly south of Bolivia, the Central and Southern Andes of Chile are the result to oblique convergence between the subducting oceanic Nazca Plate and the overriding continental South America Plate. The average direction of convergence is about $N 78^{\circ} \mathrm{E}$ with an absolute speed of $9-10 \mathrm{~cm} / \mathrm{yr}$. West to east, the Chilean Andes are herein divided into two distinct but parallel orogenic belts: 1) a fore arc zone including the Coastal Cordillera and the Central Depression, 2) the Main Cordillera with the active magmatic arc zone. In the southern part of the Chilean Andes, a foreland or back arc zone takes place around the Magellan Strait.

In Chile, the width of the Andes is about 150 to $200 \mathrm{~km}$ on average and some 4,000 km long. The highest peaks of the Main Cordillera are more than $5,000 \mathrm{~m}$ in the north to 2,000 $\mathrm{m}$ in the south. The different mountain belts trend roughly $\mathrm{N}-\mathrm{S}$ to $\mathrm{N} 10^{\circ} \mathrm{E}$, parallel to the active margin of the subduction zone. The North Chilean Andes, between $18^{\circ}$ and $27^{\circ} \mathrm{S}$, lie above the southernmost part of the normally dipping portion of the subducting Nazca Plate (Jordan and others, 1983). Between 27 and $33^{\circ} \mathrm{S}$, the Benioff Zone dips only $5^{\circ}$ and as such are not associated with an active volcanic arc in the Cordillera. Conversely, the South Chilean Andes, between $33^{\circ}$ and $47^{\circ} \mathrm{S}$, lie above a steeper east-dipping portion of the subducting Nazca Plate, which corresponds to the southern active volcanic arc. Southward, the Antarctic Plate subducts beneath the Andes with a convergence rate of $2 \mathrm{~cm} / \mathrm{yr}$, much lower than in the northern part of Chilean Andes.

North-south-trending minor ranges are cored by Paleozoic, Mesozoic and Cenozoic rocks that constitute the Coastal Cordillera, which is $30-50 \mathrm{~km}$ wide and 2,000-2,500 m high. The central batholith of the Cordillera is composed of metamorphic rocks and granites that intrude the sedimentary Paleozoic rocks. Surfaces eroded across these rocks form the Coastal Plain.

In the northern part of the Chilean Andes, the Pampa de Tamarugal represents a young tectonic depression (also known as the northern Central Depression or Longitudinal Valley) that morphologically separates the Coastal Range from the Precordillera. Extensional tectonics do not seem to have played a role in the formation of the Central Depression (Reutter and others, 1988), in contrast with coastal marine terraces that are affected by E-W extensional deformation.

From Santiago to the south, the southern Central Depression corresponds to a morphotectonic graben-like depression that developed during Miocene to Pliocene time. This depression is bounded by longitudinal faults, presently inactive. The thickness of the continental sedimentary fill in the depression (Pliocene and Quaternary deposits reach $500 \mathrm{~m}$ to 2,000 $\mathrm{m}$ ) increase progressively toward the south where marine deposits are noted.

The Main Cordillera is comprised essentially of sedimentary deposits with interbedded volcanic layers (eastern part of the Chilean Andean slope) and by volcanogenic and continental Cenozoic rocks (western part of the Chilean Andean slope). The Pliocene-Pleistocene volcanic belt runs between these two zones.

Between $26^{\circ}$ and $33^{\circ} \mathrm{S}$, where the "Cordilleras transversales" corresponds to the flat-subducting plate portion, the relief is continuous from the main Cordillera to the Coastal Cordillera and the depression lakes.

Chilean Andean subduction is characterized by a general oblique convergence vector with respect to the plate boundary and the different modes of this oblique accomodation are problematic, specially the relations between the overriding plate deformation and the subduction. Uplift of the Andean chain is shown by elevated Quaternary marine terraces. In those coastal areas closest to the trench (located about 
80-100 km to the west), the state of stress during the upper Pleistocene was extensional in an E-W direction, generating normal faulting (e.g., the Atacama fault [CH-01]). The great (M8) July 30, 1995 Antofagasta thrust earthquakes along the interplate Wadati-Benioff (subduction) zone (which is the immediate consequence of convergence between the plates) generate morphological changes, such as coastal uplift, subsidence of the outer forearc, and an elongation of the coast toward the west. At the surface, the result of such deformation is a roughly extensional E-W state of stress. Generally, the E-W extensional deformation affects marine oxygen-isotopic stage $5 \mathrm{c}$ marine deposits (80 to $105 \mathrm{ka}$ ), but kinematic studies of neotectonic faults associated with the uplifted terraces do not show many active crustal structures directly linked to earthquakes (as noted by Armijo and Thiele, 1990; Delouis 1996, 1998).

During the Quaternary, deformation was partitioned into two states of stress south of $32^{\circ} \mathrm{S}$. In the forearc sliver (Costal Cordillera, Central Depression and part of the Main Cordillera), compressional deformation ocurred with the $\sigma 1$ axis trending N-S and the $\sigma 3$ axis in the vertical direction. In the intra-arc, compressional strike-slip deformation (transpression) occurred with the $\sigma 1$ axis trending NE-SW and the $\sigma 3$ axis trending NW-SE, parallel to the Liquiñe-Ofqui fault zone (Lavenu and Cembrano, 1999).

This N-S compressional state of stress could be related to the effect of the partitioning of the deformation that generates the northward displacement of the fore arc sliver. According to Lavenu and Cembrano (1999), this N-S Quaternary compression is linked to a slow convergence regime with a weaker coupling. Different factors such as the geometry of the plate margin and/or abrupt changes in subduction geometry (e.g. buttress accomodation) can also explain this $\mathrm{N}-\mathrm{S}$ compresional tectonic regime. North of Santiago, the Quaternary state of stress of the Transversal Cordillera (between $18^{\circ} \mathrm{S}$ and $22^{\circ} \mathrm{S}$ ) and the Central Depression (between $25^{\circ} \mathrm{S}$ and $32^{\circ} \mathrm{S}$ ) is still unknown.

\section{PREPARATION OF THE DATABASE AND MAP}

This compilation shows evidence for activity on Quaternary faults in Bolivia and Chile and its offshore regions using guidelines modified from Haller and others (1993). The fault data for Bolivia were compiled by Alain Lavenu during 1996 using unpublished reports (gray literature), published literature, and recent geological investigations to describe 14 faults. The fault data for Chile were compiled by Alain Lavenu and Ricardo Thiele during 1997 using unpublished reports (gray literature), published literature, and recent geological investigations for 20 faults, which includes two complicated fault zones (the Atacama [CH-01] and the Liquiñe-Ofqui [CH-06]). No separate descriptions of Quaternary folds were made for Bolivia and Chile. Alain and Ricardo also interpreted aerial photographs and conducted limited field studies of some of these structures. Michael Machette edited the text and map data and provided guidance for the project under the International Lithosphere Program's Task Group II-2 "Major Active Faults and Folds of the World," for which he is Co-chairman (Western Hemisphere). The surface traces of the Quaternary faults in Bolivia were digitized from Alain's compiliation, which was on a planimetric base map. The surface traces of the Quaternary faults in Chile were digitized from Alain and Ricardo's compiliation, also on a planimetric base map. Offshore traces (Pacific Ocean) are based primarily on marine geophysical studies and bathymetric maps; these traces are inherently less well defined and located, and should be considered approximate.

Richard Dart used GIS (Geographic Information System) technology to produce the fault map. The traces of Quaternary faults were digitized, attributed for age, sense of slip, and line type (continuous, discontinuous, and concealed or inferred). These data were reprojected using a Labmert Conformal Conic Projection for Bolivia and a Polyconic Projection for Chile. The maps were prepared with ARC/INFO version 7.2.1 running under Solaris version 2.6 on a Unix workstation. The GIS data is scale independent, but should not be used at more than twice (more detailed) the digitized scale. Data for the fault length and average strike were generated from the ARC/INFO files.

The base-map information for the enclosed maps was taken from the Digital Chart of the World, which was created for use with ARC/INFO (copyright 1993 by the Environmental Systems Research Institute, Inc.). The Digital Chart of the World was compiled at a scale of 1:1,000,000, which is more detailed than the printed scales of the map. The Digital Chart of the World was originally developed for the United States Defense Mapping Agency (DMA) and is primarily derived from the DMA Operational Navigation Chart (ONC) Series. 


\section{AP}

The traces of Quaternary faults and folds of Bolivia and Chile were digitized from Lavenu and Thiele's compilations at 1:1,000,000 scale. This scale allows output as single-country maps at 1:1,750,000 for Bolivia and 1:4,000,000 scale for Chile using a standard a 36-inch (0.9-m) wide printer. Additionally, more detailed maps for portions of the countries can be printed at other scales $(1: 250,000$ to $1: 500,000)$ while retaining all significant digital information (see both map plates).

The fault maps show the location and style of Quaternary faults, time of most recent movement, and estimates of slip rate (as a proxy for fault activity). Although as many as five time categories of Quaternary faults can be depicted on the Western Hemisphere maps, only three categories were used in Bolivia and Chile:

Historic (generally $<450$ years),

Holocene and latest Pleistocene $(<15,000$ years or $<15 \mathrm{ka})$,

Quaternary $(<1,600,000$ years or $<1.6 \mathrm{Ma})$.

Categories for the late Quaternary ( $<130 \mathrm{ka})$ and late and middle Quaternary $(<750 \mathrm{ka})$ were not used owing to the general lack of stratigraphic and chronological control needed to make these age

differentiations. This categorical time scheme allows some flexibility in reporting between countries owing to the differing levels of investigation and abilities to date prehistoric faulting.

Three ranges of slip rates depicted by differing lines are shown on the map in order to differentiate known rates of fault activity:

$>5 \mathrm{~mm} / \mathrm{yr}$-Plate-boundary faults and subduction zones,

1-5 mm/yr - Lesser strike-slip and major extensional faults,

$<1 \mathrm{~mm} / \mathrm{yr}$-Most extensional and intraplate faults.

Most faults in Bolivia and Chile with "unknown slip rates" are drawn with the $<1-\mathrm{mm} / \mathrm{yr}$ line thickness.

\section{DATABASE}

The purpose of the database is to provide large quantities of fault data that can be readily accessed using a variety of search parameters. For this database, we anticipate that the user would want search-andretrieve capabilities from a personal computer. The user may want to sort the data by such parameters as fault name, time of most recent movement (one of three categories), slip rate (one of three categories), sense of movement, or by multiple parameters.

The process of data compilation starts with data acquisition and synthesis. In the case of faults, the compiler must determine if the structure is a simple one, or if it qualifies as having sections (increasing complexity of geometry or fault history). Then using the appropriate form, the compiler tabulates information on the fault's parameters. The forms were built in Microsoft Word for the Macintosh.

After this report is released, we will incorporate suggested changes and additions; then import the data to the computer database. Each of the fields is a potential search object. The use of a computer database program allows us to custom format the reporting of data and to collapse unused fields or notes. The basic fields are restricted to 256 characters, but we use the note option for more explanatory information (shown under comments in this report).

The fault data will be released in several forms. This open-file report constitutes a traditional hard-copy catalog (database and map) for Bolivia and Chile. The Bolivian and Chilean data will eventually be part of a larger relational computer database for the Western Hemisphere that should be available on the World Wide Web (WWW). This interactive WWW product allows the user to browse, sort, and print the data. However, we do not anticipate allowing the database to be altered using only the run-time WWW version of the database program.

\section{DEFINITION OF DATABASE TERMS}

The following terms and descriptions, which are shown in English and Spanish (when considerably different), provide data for specialized fields, most of which will be searchable when the computer database is released. In additional specialized fields, more detailed information is provided in the "Comments" section that follows some fields. If a field is empty, marked unknown, or has been deleted, no pertinent information was found in the published literature. The following description provides definitions 
of fields (in alphabetic order) and indicates where various information, if known, can be found. English language citations for references are in a traditional (USGS) format, although foreign language citations are as provided by the compilers.

Average dip General down-dip direction of the structure, where known.

Average strike The length-weighted average strike of the fault trace is reported in the northwest and northeast quadrants of the compass (i.e., $-30^{\circ}$ is $\mathrm{N} .30^{\circ} \mathrm{W}$., versus $30^{\circ}$ which is S. $30^{\circ} \mathrm{E}$. The values (i.e., $\pm 30^{\circ}$ ) that follow the strike describe the range for all vectors contained with the trace of that particular fault or collection of faults. These values are included only to provide a general impression of the sinuosity or variability in strike of the mapped structures. Some fault zones include faults with a wide variety of strikes, and thus \pm values are large.

Compiler, affiliation and date of compilation The name and affiliation of the person(s) primarily responsible for compilation or update of data presented for the structure. Also shown is the date when data were compiled for this project (e.g., January 1997).

Fault geometry This heading includes geographic information pertinent to the fault being described. The data include length, average strike, average dip, and sense of movement.

Geomorphic expression General description of the structure's geomorphic expression including information on the the presence or absence of fault scarps, offset streams, monoclines, shutter ridges, associated landslides, etc.

Historical surface faulting When the timing of most recent movement on a fault is historic, then this field(s) describes evidence for surface faulting associated with historical earthquakes. Also included is seismological information for the historical earthquake.

Length This field specifies the end-to-end length of the Quaternary-age fault (or fault section) as measured from the most distal ends of the trace. The ends of overlapping or echelon traces are projected to a line defined by the average strike and the length is then determined from those projected end points. Also shown (in parentheses) is the cumulative length of all surface traces included in the fault, fault zone, or collection of faults.

Name (Fault name or Section name) The earliest referenced name for a structure or fault section (where appropriate) generally is given preference, except in cases where a more commonly accepted name is widely used in the recent literature. "Comments" may also contain other names and references in which they are used, the geographic limits of the structure, north to south or west to east, as shown in this compilation; various geographic limits that are different than in other studies are also included. Minor changes in original name may have been made for reasons of clarity or consistency (such as segment to section) where appropriate. We have found no faults in Bolivia and Chile that justify using the term "segment", owing to a lack of precise timing information.

Number

Fault number The structure (fault) is assigned a number that is preceded by a two character abbreviation (Bolivia fault number 1 is BO-01) that is unique to each of the countries in the Western Hemisphere. References to the same structure shown in other compilations, such as $\mathrm{CH}-01$ are included in "Comments".

Fault Section number An alpha character is assigned to the northernmost or westernmost section of a fault (for example, fault $\mathrm{CH}-01$ has five sections: $\mathrm{CH}-01$ a through $\mathrm{CH}-01 \mathrm{e}$ ).

Number of sections (only used for faults with sections) Numeric value for number of sections (e.g., 2) defined in studies that do not meet the minimum requirements for segments established for this compilation. "Comments" include reference in which sections are discussed; if the term "segment" is used in the literature, an explanation of why "section" is used in the database is provided.

Recurrence interval Time interval in yr (based on historic data, calendric or calibrated radiocarbon dates), in ${ }^{14} \mathrm{C}$ yr (based on uncalibrated radiocarbon dates), or in k.y. (thousand years, based on less precise dating methods, stratigraphy, or geomorphology). Unknown is shown if there is no published recurrence interval value. Alternative published recurrence intervals, starting with that which applies to the most recent time interval, are included in "Comments." No faults in Bolivia and Chile have established recurrence intervals.

References A bibliographic citation is included for all references pertinent to each structure. Papers published in Spanish are cited in Spanish, and may not conform to USGS style. 
Section A geographic, geometric, structural portion of a fault or collection of faults that appear(s) to have a different character than adjacent portions of the fault. Typically, not enough information exists to show that this portion of the fault acts independently of adjacent portions, and thus does not qualify as a bona fide "segment" of a fault in a paleoseismic sense. There are no known faults with proven segments in Bolivia and Chile, although two faults within Chile are described as having sections. Further research is needed to document additional faults with sections or those with sections that may in fact be segments.

Section name (see Name)

Section number (see Number)

Sense of movement Includes thrust, less than $45^{\circ}$ dip; reverse, greater than $45^{\circ}$ dip; right-lateral strike slip (dextral); left-lateral strike slip (sinistral); or normal faults. For oblique slip, the principle sense of movement is followed by secondary sense (i.e., dextral, normal).

Slip rate The primary field shows an actual value or one of several slip-rate categories used for the map part of this compilation: $<1 \mathrm{~mm} / \mathrm{yr}, 1-5 \mathrm{~mm} / \mathrm{yr}$, or $>5 \mathrm{~mm} / \mathrm{yr}$. Very few faults in Bolivia and Chile have established slip rates. "Unknown" precedes the suspected slip-rate or slip rate category if no published slip rate is known. "Comments" may include a synopsis of published slip rates and pertinent documentation. Generally speaking, there are two types of slip rates. The first type is termed a "Geologic slip rate" and is derived from the age and amount of offset of surficial geologic deposits. These rates are not precise, but allow one to place broad limits on possible slip rates, and hence characterize the fault in one of the above-mentioned categories. Most slip rates from Bolivia and Chile are geologically determined. The second type of slip rate is termed a "Paleoseismic slip rate" and is derived from times of faulting events and amounts of offset of geologic datums or piercing point. This type of slip rate is more precise, but are rare owing to the extensive amount of work involved (i.e., detailed paleoseismologic studies involving trenching and numeric dating).

Fault name (see Name)

Fault number (see Number)

Synopsis and geologic setting This field provides a short summary that describes the level of study, provides a snapshot of the scope of data that follows in the database and provides a generalized perspective of the fault in terms of its regional geologic setting, amount of total offset, and general age of offset strata. Not all faults in the database have a synopsis and discussion of geologic setting.

Timing of most recent event (faulting event) The primary field shows one of the two prehistoric time categories: latest Quaternary (Holocene and latest Pleistocene, $<15 \mathrm{ka}$ ) or Quaternary ( $<1.6 \mathrm{Ma})$. This field may document historic surface faulting, although details of the earthquake related to the faulting will follow.

Type of studies: This field briefly summarizes the types of studies conducted on the fault.

\section{ACKNOWLEDGEMENTS}

This project was supported by the USGS's National Earthquake Hazards Reduction Program (NEHRP) and by the International Lithosphere Program (ILP) under Task Group II-2. Alain Lavenu's time was graciously supplied by IRD (Institut de Recherche pour le Développement, formerly ORSTOM) as part of their cooperative research program in intratropical countries. Most of IRD's investigations have been conducted at the bequest of the Bolivian and Chilean Universities; we greatly appreciate their support and continuing interest in paleoseismology and seismic-hazard assessments. Ricardo Thiele's time was graciously supplied by the Department of Geology at the University of Chile, Santiago.

\section{REFERENCES CITED ABOVE}

Armijo, R., and Thiele, R., 1990, Active faulting in Northern Chile-Ramp stacking and lateral decoupling along a subduction plate boundary?: Earth and Planetary Science Letters, v. 98, p. 40-61.

Baby, P., Hérail, G., López, J.M., López, O., Oller, J., Pareja, J., Sempéré, T., Tufiño, D., 1989. Structure de la Zone subandine de Bolivie : Influence de la géométrie des séries sédimentaires antéorogéniques sur la propagation des chevauchements. C. R. Acad. Sci., sér. 2, 309, 1717-1722.

Baby, P., Hérail, G., Salinas, R., Sempéré, T., 1992, Geometry and kinematic evolution of passive roof duplexes deduced from cross section balancing-Example from the foreland thrust system of the Southern Bolivian Subandean Zone: Tectonics, v. 11, no. 3, p. 23-536. 
Delouis, B., 1996, Subduction et déformation continentale au nord-Chili. Unpub. Doctorat thesis, Univ. Louis Pasteur, Strasbourg, France, $262 \mathrm{p}$.

Delouis B., Philip H., Dorbath L., and Cisternas A., 1998, Recent crustal deformation in the Antofagasta region (Northern Chile) and the subduction process: Geophysical Journal International, v. 132, p. 302-338.

Dumont, J.F., 1996, Neotectonics of the Subandes-Brazilian craton boundary using geomorphological data-The Marañon and Beni basins: Tectonophysics, v. 257, p. 137-151.

Haller, K.M., Machette, M.N., and Dart, R.L., 1993, Maps of Major Active Faults, Western Hemisphere; International Lithosphere Program (ILP) Project II-2: Guidelines for U.S. Database and Map: U.S. Geological Survey OpenFile Report 93-338, $45 \mathrm{p}$.

Jordan, T.E., Isacks, B.L., Allmendinger, R.W., Brewer, J.A., Ramos, V., and Ando, C.J., 1983, Andean tectonics related to geometry of subducted Nazca plate: Geological Society of America Bulletin, v. 94, p. 341-361.

Lavenu, A., 1988, Etude tectonique et néotectonique de l'Altiplano et de la Cordillère orientale des Andes boliviennes: Travaux et Documents microfichés, v. 28, 420 pp., ORSTOM, Paris.

Lavenu, A., and Marocco, R., 1984, Continental sedimentation and tectonics in a fold belt related to a subduction zone-The Central Andes (Perú-Bolivia) during the Tertiary: Bulletin du centre de Recherche et d'ExplorationProduction, Elf-Aquitaine, v. 8, no. 1, p. 57-70.

Lavenu, A., 1992, Formation and geological evolution, in Dejoux C. and Iltis A., eds, Lake Titicaca: The Netherlands, Kliwer Academic Publisher, p. 1-13.

Lavenu, A., and Cembrano, J., 1999, Compressional- and transpressional-stress pattern for Pliocene and Quaternary brittle deformation in fore arc and intra-arc zones (Andes of Central and Southern Chile): Journal of Structural Geology, v. 21, p. 1669-1691.

Martinez, C., 1980, Structure et évolution de la chaîne andine dans le nord de la Cordillère des Andes de Bolivie: Travaux et Documents, ORSTOM, no. 119, 352 pp.

Mercier, J.L., Sébrier, M., Lavenu, A., Cabrera J., Bellier, O., Dumont, J.F., and Macharé, J., 1992, Changes in the tectonic regime above a subduction zone of Andean type-The Andes of Peru and Bolivia during the PliocenePleistocene: Journal of Geophysical Research, v. 97, no. B8, p. 11,945-11,982.

Meyer, H., and Murillo, J., 1961, Sobre la geología en las provincias Aroma-Pacajes y Carangas: Departamento Nacional de Geología de Bolivia, no. 1, $47 \mathrm{pp}$.

Reutter, K.J., Giese P., Gotze H.J., Scheuber, E., Schwab, K., Schwarz G., and Wigger, P., 1988, Structures and crustal development of the Central Andes between $21^{\circ}$ and $25^{\circ} \mathrm{S}$., in H. Bahlburg, Ch., and Breitkreuz, P. Giese (eds.), The Southern Central Andes: Lecture notes in Earth Sciences, p. 231-261, Springer Verlag, Berlin.

Rodrigo, L.A., and Castaños, A., 1975, Estudio sedimentológico de las formaciones "Tiwanaku, Coniri y Kollu Kollu" del Altiplano septentrional boliviano: Sociedad Geológica Boliviana, v. 22, p. 85-126.

Sébrier, M., Mercier, J.L., Mégard, F., Laubacher, G., and Carey-Gailhardis, E., 1985, Quaternary normal and reverse faulting and the state of stress in central Andes of Southern Peru: Tectonics, v. 4, no. 7, p. 739-780. 


\section{FAULT DATABASE FOR BOLIVIA BO-01, FAULT EAST OF ACHACACHI}

FAULT NUMBER/NUMERO DE LA FALLA: BO-01

FAULT NAME/NOMBRE DE LA FALLA: East of Achacachi (informal name)/Este de Achacachi (sin nombre)

SYNOPSIS AND GEOLOGIC SETTING/SINOPSIS Y AMBIENTE GEOLOGICO: Quaternary activity described in 1981. General neotectonic field studies. This fault is more than $50 \mathrm{~km}$ long and affects glacial sediments (Quaternary moraines). Located in the piedmont of the Eastern Cordillera, on limit with the Altiplano. It is part of a Quaternary fault system that formed Lake Titicaca.

Actividad cuaternaria descrita en 1981. Estudios neotectónicos de campo generales. La falla de más de $50 \mathrm{~km}$ de largo afecta sedimentos glaciales (morenas cuaternarias). Se ubica en el piedemonte de la Cordillera Oriental, en limite con el Altiplano. Hace parte de un sistema de fallas cuaternarias que originó el Lago Titicaca.

COMPILER, AFFILIATION, \& DATE OF COMPILATION/COMPILADOR, AFILIACION Y FECHA DE COMPILACION: Alain Lavenu, IRD, France; 06-1996

TYPE OF STUDIES/TIPOS DE ESTUDIOS: Interpretation of aerial photographs and fieldwork. Fotointerpretación y trabajos de campo.

FAULT GEOMETRY/GEOMETRIA DE LA FALLA:

LENGTH/LONGITUD: $29.9 \mathrm{~km}$ (29.5 km cumulative)

AVERAGE STRIKE/RUMBO PROMEDIO: $-57.4^{\circ} \pm 11^{\circ}\left(\mathrm{N} 57.4^{\circ} \mathrm{W} \pm 11^{\circ}\right)$

AVERAGE DIP/INCLINACION PROMEDIO: Unknown angle to NE/Desconocida al NE

SENSE OF MOVEMENT/SENTIDO DE MOVIMIENTO: Normal

Comments/Comentarios: Other faults dipping SW are associated with this fault/

A esta falla estan asociadas otras fallas con buzamiento al SW.

GEOMORPHIC EXPRESSION/EXPRESION GEOMORFOLOGICA: The scarp faces towards the NE, is eroded and not very obvious.

El escarpe, hacia el NE, es erosionado, poco marcado.

SLIP RATE/TASA DE MOVIMIENTO: Unknown/desconocida

TIME OF MOST RECENT OF MOVEMENT/EDAD DEL ULTIMO MOVIMIENTO: Quaternary/Cuaternario $(<1.6 \mathrm{Ma})$

Comments/Comentarios: The fault affects Sorata moraines (younger than Purapurani Fm., 1.6 Ma) but does not affect Choqueyapu moraines (between 16 ka and more than $40 \mathrm{ka}$ ).

La falla afecta a morenas Sorata (post Fm. Purapurani, 1.6 Ma) y no afecta a morenas Choqueyapu (entre 16 ka y más de $40 \mathrm{ka}$ ).

REFERENCES/REFERENCIAS

Lavenu, A., 1981, Origine et évolution néotectonique du lac Titicaca: Revue d'Hydrobiologie Tropicale, v. 14, no. 4, p. 289-297.

Lavenu, A., 1988, Etude tectonique et néotectonique de l'Altiplano et de la Cordillère orientale des Andes boliviennes: Travaux et Documents microfichés, 28, 420 p., ORSTOM, Paris.

Lavenu, A., Bonhomme, M., Vatin-Pérignon, N., De Pachtère, P., 1989, Neogene magmatism in the Bolivian Andes between $16^{\circ}$ and $18^{\circ} \mathrm{S}$.-Stratigraphy and K-Ar geochronology: Journal of South American Earth Sciences, v. 2, p. 35-45.

Lavenu, A., Mercier, J.L., 1991, Evolution du régime tectonique de l'Altiplano et de la Cordillère orientale des Andes de Bolivie du Miocène supérieur à l'Actuel: Géodynamique, v. 6, no. 1, p. 21-55.

\section{BO-02, FAULT EAST OF PEÑAS}

FAULT NUMBER/NUMERO DE LA FALLA: BO-02

FAULT NAME/NOMBRE DE LA FALLA: East of Peñas (informal name)/Este de Peñas (sin nombre) SYNOPSIS AND GEOLOGIC SETTING/SINOPSIS Y AMBIENTE GEOLOGICO: Quaternary activity described in 1981. General neotectonic field studies. This fault puts in contact Quaternary sediments with Meso-Cenozoic basement. Located in the piedmont of the Eastern Cordillera, on margin of the Altiplano. 
Actividad cuaternaria descrita en 1981. Estudios neotectónicos de campo generales. Pone en contacto sedimentos cuaternarios fluvio-glaciales con substrato meso-cenozoico. Se ubica en el piedemonte de la Cordillera Oriental, en limite con el Altiplano

COMPILER, AFFILIATION, \& DATE OF COMPILATION/COMPILADOR, AFILIACION Y FECHA DE COMPILACION: Alain Lavenu, IRD, France; 06-1996

TYPE OF STUDIES/TIPOS DE ESTUDIOS: Fieldwork. Interpretation of aerial photographs.

Trabajos de campo. Fotointerpretación

FAULT GEOMETRY/GEOMETRIA DE LA FALLA:

LENGTH/LONGITUD: $53.6 \mathrm{~km}$ (60.7 km cumulative)

AVERAGE STRIKE/RUMBO PROMEDIO: $-46.3^{\circ} \pm 11^{\circ}\left(\mathrm{N} 46.3^{\circ} \mathrm{W} \pm 11^{\circ}\right)$

AVERAGE DIP/INCLINACION PROMEDIO: $70^{\circ} \mathrm{NE}$

SENSE OF MOVEMENT/SENTIDO DE MOVIMIENTO: Normal

Comments/Comentarios: This fault is parallel to the border of the Eastern Cordillera and can be

followed, although discontinuously, over some $40 \mathrm{~km}$.

La falla está paralela al borde de la Cordillera Oriental y aunque discontínua, se sigue sobre unos $40 \mathrm{~km}$.

GEOMORPHIC EXPRESSION/EXPRESION GEOMORFOLOGICA: Eroded scarp. In old rocks, a throw of approximately $200 \mathrm{~m}$ is distinguished. This fault displaces an erosion surface (S5) that is post $1.6 \mathrm{Ma}$. In some sectors throw is smaller, the fault affecting more recent deposits, such as the Choqueyapu moraines, dated between $16 \mathrm{ka}$ and more than $40 \mathrm{ka}$. Smaller antithetic faults of the same age exist, with dip towards the SW.

Escarpe erosionado. Un rechazo de $\sim 200 \mathrm{~m}$ se nota en rocas antiguas. Desplaza una superficie de erosión (S5) post 1.6 Ma. En algunos sectores el rechazo es menor, la falla afectando terrenos más recientes, como morenas Choqueyapu datadas entre 16 ka y mas de 40 ka. Existen fallas antitéticas de misma edad, más pequeñas, con buzamiento hacia el SW.

SLIP RATE/TASA DE MOVIMIENTO: Unknown/desconocida

Comments/Comentarios: About $0.125 \mathrm{~mm} / \mathrm{yr}$ according to profile./Ca. $0.125 \mathrm{~mm} /$ año según perfil.

TIME OF MOST RECENT OF MOVEMENT/EDAD DEL ULTIMO MOVIMIENTO: (<15 ka)

Comments/Comentarios: This fault, like the Kenko fault zone [BO-03] in La Paz, presents successive and different magnitude movements. A vertical apparent displacement of hectometric magnitude and post 1.6 Ma exists (lower Pleistocene-middle Pleistocene?), as well as a recent (late

Pleistocene-Holocene) apparent displacement of decimeters to meters magnitude.

Esta falla, como el sistema de Falla Kenko [BO-03] de La Paz, tiene movimientos sucesivos y de magnitud diferente. Existe un desplazamiento vertical aparente post 1.6 Ma (Pleistoceno inferior-

Pleistoceno medio ?) de magnitud hectométrica, y un desplazamiento aparente reciente (Pleistoceno superior-Holoceno) decimétrico a métrico.

REFERENCES/REFERENCIAS

Lavenu, A., 1981, Origine et évolution néotectonique du lac Titicaca: Revue d'Hydrobiologie Tropicale, v. 14, no. 4, p. 289-297.

Lavenu, A., 1988, Etude tectonique et néotectonique de l'Altiplano et de la Cordillère orientale des Andes boliviennes: Travaux et Documents microfichés, v. 28, 420 pp., ORSTOM, Paris.

Lavenu, A., Mercier, J.L., 1991, Evolution du régime tectonique de l'Altiplano et de la Cordillère orientale des Andes de Bolivie du Miocène supérieur à l'Actuel: Géodynamique, v. 6, no. 1, p. 21-55.

\section{BO-03, KENKO FAULT ZONE}

FAULT NUMBER/NUMERO DE LA FALLA: BO-03

FAULT NAME/NOMBRE DE LA FALLA: Kenko zone/zona de Kenko

SYNOPSIS AND GEOLOGIC SETTING/SINOPSIS Y AMBIENTE GEOLOGICO: First described in 1962;

Quaternary activity described in 1978. General neotectonic field studies. This fault puts in contact

Quaternary fluvio-glacial sediments with Pliocene fluvio-lacustrine ones. This fault displaces the topographic surface of the Altiplano between La Paz and the airport. The fault belongs to a complex normal fault system. The Kenko fault zone comprises the main trace, approximately trending $N 130^{\circ} \mathrm{E}$. Associated with branches trending $\mathrm{N} 110^{\circ} \mathrm{E}, \mathrm{N} 150^{\circ} \mathrm{E}$ and $\mathrm{N} 40-70^{\circ} \mathrm{E}$. These correspond to the 
reactivation of deep strike-slip faults resulting from a Riedel-type shear system developed during the compressive terminal-Pliocene event ( $\sigma 1$ approx. E-W about $2 \mathrm{Ma}$ ). The main fault, trending $\mathrm{N} 130^{\circ} \mathrm{E}$, corresponds to the main shear. The $\mathrm{N} 110^{\circ} \mathrm{E}$ direction strands correspond to Riedel's R fractures, the $\mathrm{N} 50^{\circ} \mathrm{E}$ ones to the R' ones and the $\mathrm{N} 150^{\circ} \mathrm{E}$ strands to Skampton's $\mathrm{P}$ fractures.

Primera descripción en 1962; actividad cuaternaria descrita en 1978. Estudios neotectónicos de campo generales. Pone en contacto sedimentos cuaternarios fluvio-glaciales con sedimentos pliocenos fluviolacustres. Desplaza la superficie topográfica del Altiplano entre La Paz y el aeropuerto. Pertenece a un sistema de fallas normales complejo. El sistema de falla Kenko conforma la traza principal de dirección $\mathrm{N} 130^{\circ} \mathrm{E}$. Esta traza está asociada a ramas de direcciones $\mathrm{N} 110^{\circ} \mathrm{E}, \mathrm{N} 150^{\circ} \mathrm{E}$, y N $40-70^{\circ} \mathrm{E}$. Corresponden en profundidad a la reactivación de fallas de rumbo resultantes de un sistema de cizalle de tipo Riedel que se desarrolló durante el evento compresivo del Plioceno terminal ( $\sigma 1 \mathrm{E}-\mathrm{W}$ about $2 \mathrm{Ma}$ ). La falla principal de dirección $\mathrm{N} 130^{\circ} \mathrm{E}$ corresponde al cizallamiento principal. Las ramas de dirección $\mathrm{N} 110^{\circ} \mathrm{E}$ corresponden a las fracturas $R$ de Riedel, las ramas de dirección $N 50^{\circ} \mathrm{E}$ a $\mathrm{R}^{\prime}$ y las ramas $N 150^{\circ} \mathrm{E}$ a las fracturas $P$ de Skampton.

COMPILER, AFFILIATION, \& DATE OF COMPILATION/COMPILADOR, AFILIACION Y FECHA DE COMPILACION: Alain Lavenu, IRD, France; 06-1996

TYPE OF STUDIES/TIPOS DE ESTUDIOS: Field work, microtectonic analysis, and interpretation of aerial photographs.

Trabajos de campo, análisis microtectónico, y fotointerpretación.

FAULT GEOMETRY/GEOMETRIA DE LA FALLA:

LENGTH/LONGITUD: $11.4 \mathrm{~km}$ (17.2 km cumulative)

AVERAGE STRIKE/RUMBO PROMEDIO: $-35.7^{\circ} \pm 8^{\circ}\left(\mathrm{N} 35.7^{\circ} \mathrm{W} \pm 8^{\circ}\right)$

AVERAGE DIP/INCLINACION PROMEDIO: $75^{\circ} \mathrm{SE}$

SENSE OF MOVEMENT/SENTIDO DE MOVIMIENTO: Normal-sinistral

Comments/Comentarios: Even though there are no fault striae on the actual fault plane, the Kenko fault zone belongs to system of normal faults formed by N-S extension (cf. Kenko and Amachuma [BO-05] faults).

Aunque no se nota la existencia de estrias en el plano mismo, la falla Kenko pertenece a un sistema de fallas normales debido a una extensión de dirección N-S (cf. fallas Kenko y Amachuma [BO-05]).

GEOMORPHIC EXPRESSION/EXPRESION GEOMORFOLOGICA: Scarp well preserved in Quaternary fluvio-glacial sediments (post $1.6 \mathrm{Ma}$ ). The topographic surface throw is meter size. The fault plane that displaces this topographic surface has less vegetation than other parts of the area, indicating relatively recent movements.

Escarpe bien conservado en sedimentos cuaternarios fluvio-glaciales (post $1.6 \mathrm{Ma}$ ). El rechazo de la superficie topográfica es métrico. El plano de falla que desplaza esta superficie topográfica presenta menor vegetación que otras partes de la zona, indicando movimientos relativamente recientes.

SLIP RATE/TASA DE MOVIMIENTO: $<0.2 \mathrm{~mm} / \mathrm{yr}$ ( $<0.2 \mathrm{~mm} / \mathrm{año})$

Comments/Comentarios: Rate estimated according to throw from geological profile.

Estimada según rechazo en perfil geológico

TIME OF MOST RECENT OF MOVEMENT/EDAD DEL ULTIMO MOVIMIENTO: Quaternary/Cuaternario $(<1.6 \mathrm{Ma})$

REFERENCES/REFERENCIAS

Dobrovolny, E., 1962, Geología del Valle de La Paz: Depto. Nac. Geol. Minist. Min. Petr. Bol. No. 3, esp. 153 p., La Paz.

Lavenu, A., 1978, Néotectonique des sédiments plio-quaternaires de l'Altiplano bolivien (région de La Paz, Ayo Ayo, Umala): Cah. ORSTOM, sér. Géol., v. X, no. 1, p. 115-126.

Lavenu, A., 1981, Origine et évolution néotectonique du lac Titicaca: Revue d'Hydrobiologie Tropicale, v. 14, no. 4, p. 289-297.

Lavenu, A., 1988, Etude tectonique et néotectonique de l'Altiplano et de la Cordillère orientale des Andes boliviennes: Travaux et Documents microfichés, v. 28, 420 pp., ORSTOM, Paris.

Lavenu, A., Mercier, J.L., 1991, Evolution du régime tectonique de l'Altiplano et de la Cordillère orientale des Andes de Bolivie du Miocène supérieur à l'Actuel: Géodynamique, v. 6, no. 1, p. 21-55. 


\section{BO-04, LLOJETA FAULT}

FAULT NUMBER/NUMERO DE LA FALLA: BO-04

FAULT NAME/NOMBRE DE LA FALLA: Llojeta

SYNOPSIS AND GEOLOGIC SETTING/SINOPSIS Y AMBIENTE GEOLOGICO: First described in 1962;

Quaternay activity described in 1978. General neotectonic field studies. Puts Quaternary fluvio-glacial

sediment in contact with Pliocene fluvio-lacustral sediment. Fault displaces the present surface of the

Altiplano. It belongs to the Kenko fault zone [BO-03] and corresponds to a $\mathrm{N} 110^{\circ} \mathrm{E}$ trending branch.

The direction of the Llojeta fault is parallel to the direction of strike-slip faults resulting from a shear

system. It corresponds to the reactivation of an R segment in a Riedel type system developed during the terminal-Pliocene compressive event ( $\sigma 1$ approx. E-W about $2 \mathrm{Ma})$.

Primera descripción en 1962; actividad cuaternaria descrita en 1978. Estudios neotectónicos de campo generales. Pone en contacto sedimentos cuaternarios fluvio-glaciales con sedimentos pliocenos fluviolacustres. Desplaza la superficie actual del Altiplano. Pertenece al sistema de la falla Kenko y

corresponde a una rama de dirección $\mathrm{N} 110^{\circ} \mathrm{E}$. La dirección de la falla Llojeta se superpone a la dirección de las fallas de rumbo resultantes de un sistema de cizalle. Corresponde a la reactivación de las fracturas $\mathrm{R}$ en sistema de tipo Riedel que se desarrollaron durante el evento compresivo del Plioceno terminal (2 $\mathrm{Ma} ; \sigma 1 \mathrm{E}-\mathrm{W})$.

COMPILER, AFFILIATION, \& DATE OF COMPILATION/COMPILADOR, AFILIACION Y FECHA DE COMPILACION: Alain Lavenu, IRD, France; 06-1996

TYPE OF STUDIES/TIPOS DE ESTUDIOS: Field work, microtectonic analysis, and interpretation of aerial photographs.

Trabajos de campo, análisis microtectónico, y fotointerpretación.

FAULT GEOMETRY/GEOMETRIA DE LA FALLA:

LENGTH/LONGITUD: $12.7 \mathrm{~km}$ (23.0 km cumulative)

AVERAGE STRIKE/RUMBO PROMEDIO: $-50.2^{\circ} \pm 16^{\circ}\left(\mathrm{N} 50.2^{\circ} \mathrm{W} \pm 16^{\circ}\right)$

AVERAGE DIP/INCLINACION PROMEDIO: $75^{\circ} \mathrm{NE}$

SENSE OF MOVEMENT/SENTIDO DE MOVIMIENTO: Normal-dextral

Comments/Comentarios: Tension gashes have been observed in slope debris. A natural perpendicular section permits the observation of normal fault deformation, repeated during all the Quaternary. The throw diminishes from $80 \mathrm{~m}$ in lower Quaternary sediment to only some decimeters (displacement of present topographic surface). Presence of some striae on fault plane or on pebbles. Microtectonic analysis allows computation a NNE-SSW extensional direction, with $\sigma 3 \mathrm{~N} 14^{\circ} \mathrm{E}$.

En escombros de pendiente se encuentran grietas de extensión. Un corte perpendicular natural permite observar una deformación en falla normal repetida durante todo el Cuaternario. El rechazo diminuye desde unos $80 \mathrm{~m}$ en el Cuaternario antiguo hasta unos decimetros (desplazamiento de la superficie topográfica). Presencia de unas escasas estrias en plano de falla o sobre guijarros. El análisis microtectónico permite calcular una dirección de extensión NNE-SSW con $\sigma 3$ de dirección $\mathrm{N} 14^{\circ} \mathrm{E}$.

GEOMORPHIC EXPRESSION/EXPRESION GEOMORFOLOGICA: Scarp well preserved on Quaternary fluvio-glacial sediment.

Escarpe bien conservado en sedimento cuaternarios fluvio-glaciales.

SLIP RATE/TASA DE MOVIMIENTO: $<0.2 \mathrm{~mm} / \mathrm{yr}(<0.2 \mathrm{~mm} / \mathrm{año})$

Comments/Comentarios: Rate of $0.06 \mathrm{~mm} / \mathrm{yr}$ estimated from the geological section.

Tasa de $0.06 \mathrm{~mm} / \mathrm{año} \mathrm{estimado} \mathrm{a} \mathrm{partir} \mathrm{del} \mathrm{perfil} \mathrm{geológico.}$

TIME OF MOST RECENT OF MOVEMENT/EDAD DEL ULTIMO MOVIMIENTO: Quaternary/Cuaternario $(<1.6 \mathrm{Ma})$

Comments/Comentarios: The activity could be more recent, possibly postglacial.

El movimiento podría ser mas reciente, de edad post-glacial.

REFERENCES/REFERENCIAS

Dobrovolny, E., 1962, Geología del Valle de La Paz: Depto. Nac. Geol. Minist. Min. Petr. Bol. n`3, esp. 153 p., La Paz.

Lavenu, A., 1978, Néotectonique des sédiments plio-quaternaires de l'Altiplano bolivien (région de La Paz, Ayo Ayo, Umala): Cah. ORSTOM, sér. Géol., v. X, no. 1, p. 115-126. 
Lavenu, A., 1981, Origine et évolution néotectonique du lac Titicaca: Revue d'Hydrobiologie Tropicale, v. 14, no. 4, p. 289-297.

Lavenu, A., 1988, Etude tectonique et néotectonique de l'Altiplano et de la Cordillère orientale des Andes boliviennes: Travaux et Documents microfichés, v. 28, 420 pp., ORSTOM, Paris.

Lavenu, A., Mercier, J.L., 1991, Evolution du régime tectonique de l'Altiplano et de la Cordillère orientale des Andes de Bolivie du Miocène supérieur à l'Actuel: Géodynamique, v. 6, no. 1, p. 21-55.

\section{BO-05, AMACHUMA FAULT}

\section{FAULT NUMBER/NUMERO DE LA FALLA: BO-05}

FAULT NAME/NOMBRE DE LA FALLA: Amachuma

SYNOPSIS AND GEOLOGIC SETTING/SINOPSIS Y AMBIENTE GEOLOGICO: Quaternary activity described in 1978. General neotectonic field studies. This fault puts Quaternary fluvio-glacial sediment in contact with Pliocene fluvio-lacustrine sediment. It has a N125-135 $\mathrm{E}$ direction, just as Kenko fault

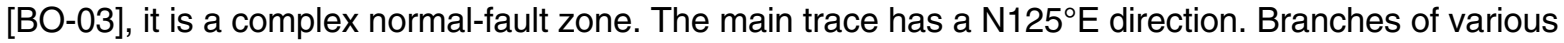
directions are associated: $\mathrm{N} 110^{\circ} \mathrm{E}, \mathrm{N} 150^{\circ} \mathrm{E}$ and $\mathrm{N} 50^{\circ} \mathrm{E}$. In depth, they correspond to the reactivation of strike-slip faults resulting of a Riedel-type shear system, developed during the uppermost-Pliocene compressive event ( $\sigma 1$ approx. E-W about $2 \mathrm{ma}$ ). The main fault, trending $\mathrm{N} 125^{\circ} \mathrm{E}$, corresponds to the main shear. The $\mathrm{N} 110^{\circ} \mathrm{E}$ strands correspond to Riedel's R fractures, the N50 $\mathrm{E}$ strands to R' fractures and the $\mathrm{N} 150^{\circ} \mathrm{E}$ strands to Skampton's $\mathrm{P}$ fractures.

Actividad cuaternaria descrita en 1978. Estudios neotectónicos de campo generales. Esta falla pone en contacto sedimentos cuaternarios fluvio-glaciales con sedimentos fluviolacustres del Plioceno. Tiene una dirección $\mathrm{N} 125-135^{\circ} \mathrm{E}$. Como para la falla Kenko [BO-03], se trata de un sistema complejo de fallas normales. La traza principal es de dirección $\mathrm{N}_{12} 5^{\circ} \mathrm{E}$. Están asociadas ramas de varias direcciones:

$\mathrm{N} 110^{\circ} \mathrm{E}, \mathrm{N} 150^{\circ} \mathrm{E}$ y $\mathrm{N} 50^{\circ} \mathrm{E}$. Corresponden en profundidad a la reactivación de fallas de rumbo resultantes de un sistema de cizalle de tipo Riedel que se desarrolló durante el evento compresivo del Plioceno terminal (2 Ma; $\sigma 1 \mathrm{E}-\mathrm{W}$ ). La falla principal de dirección $\mathrm{N} 125^{\circ} \mathrm{E}$ corresponde al cizallamiento principal. Los segmentos de dirección $\mathrm{N} 110^{\circ} \mathrm{E}$ corresponden a las fracturas R' de Riedel, los segmentos de dirección $N 50^{\circ} \mathrm{E}$ a R' y los segmentos $\mathrm{N} 150^{\circ} \mathrm{E}$ a las fracturas $\mathrm{P}$ de Skampton.

COMPILER, AFFILIATION, \& DATE OF COMPILATION/COMPILADOR, AFILIACION Y FECHA DE COMPILACION: Alain Lavenu, IRD, France; 06-1996

TYPE OF STUDIES/TIPOS DE ESTUDIOS: Field work, microtectonic analysis, and interpretation of aerial photographs.

Trabajos de campo, análisis microtectónico, y fotointerpretación.

FAULT GEOMETRY/GEOMETRIA DE LA FALLA:

LENGTH/LONGITUD: $40.0 \mathrm{~km}$ (66.5 km cumulative)

AVERAGE STRIKE/RUMBO PROMEDIO: $-45.6^{\circ} \pm 15^{\circ}\left(\mathrm{N} 45.6^{\circ} \mathrm{W} \pm 15^{\circ}\right)$

AVERAGE DIP/INCLINACION PROMEDIO: $70^{\circ} \mathrm{E}$

SENSE OF MOVEMENT/SENTIDO DE MOVIMIENTO: Normal-sinistral

Comments/Comentarios: Presence of striae. A microtectonic analysis permits computation of a N-S

extension with $\sigma 3$ trending $\mathrm{N} 175^{\circ} \mathrm{E}$.

Presencia de estrias. El análisis microtectónico permite calcular una extensión N-S con $\sigma 3$ de dirección $\mathrm{N} 175^{\circ} \mathrm{E}$.

GEOMORPHIC EXPRESSION/EXPRESION GEOMORFOLOGICA: Scarp/escarpe

SLIP RATE/TASA DE MOVIMIENTO: Unknown/desconocida

TIME OF MOST RECENT OF MOVEMENT/EDAD DEL ULTIMO MOVIMIENTO: Quaternary/Cuaternario $(<1.6 \mathrm{Ma})$

\section{REFERENCES/REFERENCIAS}

Lavenu, A., 1978, Néotectonique des sédiments plio-quaternaires de l'Altiplano bolivien (région de La Paz, Ayo Ayo, Umala): Cah. ORSTOM, sér. Géol., v. X, no. 1, p. 115-126.

Lavenu, A., 1988, Etude tectonique et néotectonique de l'Altiplano et de la Cordillère orientale des Andes boliviennes: Travaux et Documents microfichés, v. 28, 420 pp., ORSTOM, Paris. 


\section{BO-06, QUEBRADA MINASA FAULT}

FAULT NUMBER/NUMERO DE LA FALLA: BO-06

FAULT NAME/NOMBRE DE LA FALLA: Quebrada Minasa

SYNOPSIS AND GEOLOGIC SETTING/SINOPSIS Y AMBIENTE GEOLOGICO: Quaternary activity described in 1977. General neotectonic field studies. It corresponds to a zone of several parallel faults, most of them dipping towards the south, and antithetic ones dipping towards the NE. The main Minasa fault affects Quaternary glacial (Sorata moraines, post 1.6 Ma) and interglacial (Purapurani Fm., 1.6 Ma) sediment. Deformation before deposition of the Sorata Formation is more important (hectometric throw) than deformation post Sorata Formation.

Actividad cuaternaria descrita en 1977. Estudios neotectónicos de campo generales. Se trata de un sistema de varias fallas paralelas, la mayoria con buzamiento al sur y otras antitéticas con buzamiento al NE. La falla principal Minasa afecta sedimentos glaciales cuaternarios (morena Sorata post 1.6 Ma) y interglaciales (Formación Purapurani, 1.6 Ma). La deformación ante Fm. Sorata es más importante (rechazo hectométrico) que la deformación post Fm. Sorata (rechazo métrico).

COMPILER, AFFILIATION, \& DATE OF COMPILATION/COMPILADOR, AFILIACION Y FECHA DE COMPILACION: Alain Lavenu, IRD, France; 06-1996

TYPE OF STUDIES/TIPOS DE ESTUDIOS: Fieldwork, microtectonic analysis, interpretation of aerial photographs.

Trabajos de campo, análisis microtectónico, y fotointerpretación.

FAULT GEOMETRY/GEOMETRIA DE LA FALLA:

LENGTH/LONGITUD: $7.7 \mathrm{~km}$ (12.9 km cumulative)

AVERAGE STRIKE/RUMBO PROMEDIO: $-70.2^{\circ} \pm 14^{\circ}\left(\mathrm{N} 70.2^{\circ} \mathrm{W} \pm 14^{\circ}\right)$

AVERAGE DIP/INCLINACION PROMEDIO: $85^{\circ} \mathrm{S}$

SENSE OF MOVEMENT/SENTIDO DE MOVIMIENTO: Normal-dextral

Comments/Comentarios: The antithetic faults dipping toward the $\mathrm{N}$ present a normal-sinistral sense of movement. The whole set of faults corresponds to NNE-SSW extension. The presence of striae permits computation of a $\sigma 3$ stress tensor trending $\mathrm{N} 201^{\circ} \mathrm{E}$.

Las fallas antitéticas con inclinación hacia el norte tienen un sentitido de movimiento normal sinestral. El conjunto corresponde a una extensión NNE-SSW. La presencia de estrias permite calcular un tensor de esfuerzo $\sigma 3$ de dirección $\mathrm{N} 201^{\circ} \mathrm{E}$.

GEOMORPHIC EXPRESSION/EXPRESION GEOMORFOLOGICA: Eroded scarp. Escarpe erosionado

SLIP RATE/TASA DE MOVIMIENTO: Unknown, probably $<0.2 \mathrm{~mm} / \mathrm{yr}$. Desconocida, probablemente $<0.2 \mathrm{~mm} / \mathrm{año}$

Comments/Comentarios: Rate could be as much as $0.15 \mathrm{~mm} / \mathrm{yr}$ (dextral), as determined from the fault throw.

Podria alcanzar $0.15 \mathrm{~mm} / a n ̃ o ~(d e x t r a l)$ medido a partir del rechazo de la falla.

TIME OF MOST RECENT OF MOVEMENT/EDAD DEL ULTIMO MOVIMIENTO: Quaternary/Cuaternario $(<1.6 \mathrm{Ma})$

REFERENCES/REFERENCIAS

Lavenu, A., 1977, Neotectónica de los sedimentos plio-cuaternarios de la cuenca de La Paz (Bolivia): Rev. Geoci., v. 1, no. 1, p. 31-56, La Paz.

Lavenu, A., 1988, Etude tectonique et néotectonique de l'Altiplano et de la Cordillère orientale des Andes boliviennes: Travaux et Documents microfichés, v. 28, 420 pp., ORSTOM, Paris.

Lavenu, A., Mercier, J.L., 1991, Evolution du régime tectonique de l'Altiplano et de la Cordillère orientale des Andes de Bolivie du Miocène supérieur à l'Actuel: Géodynamique, v. 6, no. 1, p. 21-55.

\section{BO-07, ESCOMA FAULT}

FAULT NUMBER/NUMERO DE LA FALLA: BO-07

FAULT NAME/NOMBRE DE LA FALLA: Escoma (informal name)/(sin nombre)

SYNOPSIS AND GEOLOGIC SETTING/SINOPSIS Y AMBIENTE GEOLOGICO: Quaternary activity not described. General neotectonic field studies. The fault affects Quaternary topographic surface of lacustrine terraces. 
Actividad cuaternaria no descrita. Estudios neotectónicos de campo generales. La falla afecta la superficie topográficade terrazas lacustres cuaternarias.

COMPILER, AFFILIATION, \& DATE OF COMPILATION/COMPILADOR, AFILIACION Y FECHA DE COMPILACION: Alain Lavenu, IRD, France; 06-1996

TYPE OF STUDIES/TIPOS DE ESTUDIOS: Fieldwork and interpretation of aerial photographs.

Trabajos de campo y fotointerpretación.

FAULT GEOMETRY/GEOMETRIA DE LA FALLA:

LENGTH/LONGITUD: $26.6 \mathrm{~km}$ (26.8 km cumulative)

AVERAGE STRIKE/RUMBO PROMEDIO: $-43.2^{\circ} \pm 7^{\circ}\left(\mathrm{N} 43.2^{\circ} \mathrm{W} \pm 7^{\circ}\right)$

AVERAGE DIP/INCLINACION PROMEDIO: Unknown angle to the W/Desconocida al W

SENSE OF MOVEMENT/SENTIDO DE MOVIMIENTO: Normal

Comments/Comentarios:

GEOMORPHIC EXPRESSION/EXPRESION GEOMORFOLOGICA: Eroded scarp

Escape erodado.

SLIP RATE/TASA DE MOVIMIENTO: Unknown/desconocida.

TIME OF MOST RECENT OF MOVEMENT/EDAD DEL ULTIMO MOVIMIENTO: Quaternary/Cuaternario $(<1.6 \mathrm{Ma})$

REFERENCES/REFERENCIAS

Lavenu, A., 1988, Etude tectonique et néotectonique de l'Altiplano et de la Cordillère orientale des Andes boliviennes: Travaux et Documents microfichés, v. 28, 420 pp., ORSTOM, Paris.

Lavenu, A., 1992, Formation and geological evolution, in Dejoux C. and Iltis A., eds, Lake Titicaca: The Netherlands, Kliwer Academic Publisher, p. 1-13.

\section{BO-08, VIACHA FAULT ZONE}

FAULT NUMBER/NUMERO DE LA FALLA: BO-08

FAULT NAME/NOMBRE DE LA FALLA: Viacha zone (informal name)/zona de Viacha (sin nombre)

SYNOPSIS AND GEOLOGIC SETTING/SINOPSIS Y AMBIENTE GEOLOGICO: Quaternary activity described in 1977. General neotectonic field studies. The faults affect Quaternary sediment.

Actividad cuaternaria descrita en 1977. Estudios neotectónicos de campo generales. Las fallas afectan sedimento cuaternarios.

COMPILER, AFFILIATION, \& DATE OF COMPILATION/COMPILADOR, AFILIACION Y FECHA DE COMPILACION: Alain Lavenu, IRD, France; 06-1996

TYPE OF STUDIES/TIPOS DE ESTUDIOS: Fieldwork and interpretation of aerial photographs.

Trabajos de campo y fotointerpretación.

FAULT GEOMETRY/GEOMETRIA DE LA FALLA:

LENGTH/LONGITUD: $16.7 \mathrm{~km}$ (14.7 km cumulative)

AVERAGE STRIKE/RUMBO PROMEDIO: $31.5 \pm 32^{\circ}$ (N31.5 $\left.{ }^{\circ} \pm 32^{\circ}\right)$

AVERAGE DIP/INCLINACION PROMEDIO: Unknown angle to the SE/desconocida al SE

SENSE OF MOVEMENT/SENTIDO DE MOVIMIENTO: Normal

Comments/Comentarios: Other faults dipping towards NW are associated with this fault.

A esta falla estan asociadas otras fallas con buzamiento al NW.

GEOMORPHIC EXPRESSION/EXPRESION GEOMORFOLOGICA: No apparent morphological

expression on the last Quaternary deposits of the Altiplano surface. Fault emplacements determined using surface electrical geophysical methods.

Sin expresión morfológica aparente en los últimos depositos Cuaternarios de la superficie del Altiplano.

Fallas determinadas con métodos geofísicos eléctricos de superficie.

SLIP RATE/TASA DE MOVIMIENTO: Unknown/desconocida.

TIME OF MOST RECENT OF MOVEMENT/EDAD DEL ULTIMO MOVIMIENTO: Quaternary/Cuaternario $(<1.6 \mathrm{Ma})$

REFERENCES/REFERENCIAS

Bles, J.L., 1977, Plan de desarrollo de la Ciudad de La Paz: HAM La Paz. Consultoras, BRGM-BCEOM-Prudencio Claros y Asociados, Inf. Geol. $\mathrm{n}^{\circ}$ 7, $34 \mathrm{p}$.

Lavenu, A., 1988, Etude tectonique et néotectonique de l'Altiplano et de la Cordillère orientale des Andes boliviennes: Travaux et Documents microfichés, v. 28, 420 pp., ORSTOM, Paris. 


\section{BO-09, AYO AYO FAULT}

FAULT NUMBER/NUMERO DE LA FALLA: BO-09

FAULT NAME/NOMBRE DE LA FALLA: Ayo Ayo (informal name/sin nombre)

SYNOPSIS AND GEOLOGIC SETTING/SINOPSIS Y AMBIENTE GEOLOGICO: Quaternary activity described in 1971. General neotectonic field studies. It corresponds to an antithetic fault zone. Actividad cuaternaria descrita en 1971. Estudios neotectónicos de campo generales. Se trata de un sistema de fallas antitéticas.

COMPILER, AFFILIATION, \& DATE OF COMPILATION/COMPILADOR, AFILIACION Y FECHA DE COMPILACION: Alain Lavenu, IRD, France, 06-1996

TYPE OF STUDIES/TIPOS DE ESTUDIOS: Fieldwork, microtectonic analysis, and interpretation of aerial photographs.

Trabajos de campo, análisis microtectónico, y fotointerpretación.

FAULT GEOMETRY/GEOMETRIA DE LA FALLA:

LENGTH/LONGITUD: $14.6 \mathrm{~km}$ (19.4 km cumulative)

AVERAGE STRIKE/RUMBO PROMEDIO: $-53.6^{\circ} \pm 5^{\circ}\left(\mathrm{N} 53.6^{\circ} \mathrm{W} \pm 5^{\circ}\right)$

AVERAGE DIP/INCLINACION PROMEDIO: $70^{\circ} \mathrm{N}$

SENSE OF MOVEMENT/SENTIDO DE MOVIMIENTO: Normal-dextral

Comments/Comentarios: Several parallel and minor Quaternary antithetic faults adjoin (dip $60^{\circ} \mathrm{S}$ ). They

correspond to NNW-SSE extension. A stress tensor with $\sigma 3 \mathrm{~N} 348^{\circ} \mathrm{E}$ was determined through

microtectonic analysis of the striae.

Se adjuntan varias fallas antitéticas cuaternarias paralelas y menores (buzamiento $60^{\circ} \mathrm{S}$ ). Corresponden a una extensión NNW-SSE. El análisis microtectónico de las estrias permite calcular un tensor de esfuerzo $\sigma 3 \mathrm{~N} 348^{\circ} \mathrm{E}$.

GEOMORPHIC EXPRESSION/EXPRESION GEOMORFOLOGICA: These faults don't have morphological expression. An erosion surface, posterior to the extensive tectonic movement, does not record any scarps.

Estas fallas no tienen expresión morfológica. Una superficie de erosión, posterior al moviemiento tectónico en falla normal, no deja testimonios de escarpes.

SLIP RATE/TASA DE MOVIMIENTO: Unknown/desconocida.

TIME OF MOST RECENT OF MOVEMENT/EDAD DEL ULTIMO MOVIMIENTO: Quaternary/Cuaternario $(<1.6 \mathrm{Ma})$

REFERENCES/REFERENCIAS

Hoffstetter, R., Martinez, C., Munoz-Reyes, J., et Tomasi P., 1971, Le gisement d'Ayo Ayo (Bolivie), une succession stratigraphique Pliocène-Pléistocène datée par des mammifères: Comptes Rendus de l'Académie des Sciences, Paris, t. 273, p. 2472-2475.

Lavenu, A., 1988, Etude tectonique et néotectonique de l'Altiplano et de la Cordillère orientale des Andes boliviennes: Travaux et Documents microfichés, v. 28, 420 pp., ORSTOM, Paris.

Lavenu, A., Mercier, J.L., 1991, Evolution du régime tectonique de l'Altiplano et de la Cordillère orientale des Andes de Bolivie du Miocène supérieur à l'Actuel: Géodynamique, v. 6, no. 1, p. 21-55.

\section{BO-10, CUENCA DE CHARAÑA FAULT}

FAULT NUMBER/NUMERO DE LA FALLA: BO-10

FAULT NAME/NOMBRE DE LA FALLA: Cuenca de Charaña (informal name/sin nombre)

SYNOPSIS AND GEOLOGIC SETTING/SINOPSIS Y AMBIENTE GEOLOGICO: Quaternary activity described in 1988. General neotectonic field studies.

Actividad cuaternaria descrita en 1988. Estudios neotectónicos de campo generales.

COMPILER, AFFILIATION, \& DATE OF COMPILATION/COMPILADOR, AFILIACION Y FECHA DE

COMPILACION: Alain Lavenu, IRD, France; 06-1996

TYPE OF STUDIES/TIPOS DE ESTUDIOS: Fieldwork/trabajos de campo.

FAULT GEOMETRY/GEOMETRIA DE LA FALLA:

LENGTH/LONGITUD: $26.7 \mathrm{~km}$ (26.9 km cumulative)

AVERAGE STRIKE/RUMBO PROMEDIO: $-37.3^{\circ} \pm 14^{\circ}\left(\mathrm{N} 37.3^{\circ} \mathrm{W} \pm 14^{\circ}\right)$ 
AVERAGE DIP/INCLINACION PROMEDIO: Unknown angle to the E/Desconocida, al E.

SENSE OF MOVEMENT/SENTIDO DE MOVIMIENTO: Normal

GEOMORPHIC EXPRESSION/EXPRESION GEOMORFOLOGICA: Eroded scarp, the topographic

surface throw is meter size in some places. The fault's throw is of several tens of meters.

Escarpe erosionado, con rechazo de la superficie topográfica a veces métrico. El rechazo de la falla es de varias decenas de metros.

SLIP RATE/TASA DE MOVIMIENTO: Unknown/desconocida

TIME OF MOST RECENT OF MOVEMENT/EDAD DEL ULTIMO MOVIMIENTO: Quaternary/Cuaternario $(<1.6 \mathrm{Ma})$

REFERENCES/REFERENCIAS

Lavenu, A., 1988, Etude tectonique et néotectonique de l'Altiplano et de la Cordillère orientale des Andes

boliviennes: Travaux et Documents microfichés, v. 28, 420 pp., ORSTOM, Paris.

\section{BO-11, TUNARI FAULT}

FAULT NUMBER/NUMERO DE LA FALLA: BO-11

FAULT NAME/NOMBRE DE LA FALLA: Tunari (informal name/sin nombre)

SYNOPSIS AND GEOLOGIC SETTING/SINOPSIS Y AMBIENTE GEOLOGICO: Quaternary activity described in 1988. General neotectonic field studies.

Actividad cuaternaria descrita en 1988. Estudios neotectónicos de campo generales.

COMPILER, AFFILIATION, \& DATE OF COMPILATION/COMPILADOR, AFILIACION Y FECHA DE COMPILACION: Alain Lavenu, IRD, France; 06-1996

TYPE OF STUDIES/TIPOS DE ESTUDIOS: Fieldwork and microstructural analysis.

Trabajos de campo y análisis microestructural.

FAULT GEOMETRY/GEOMETRIA DE LA FALLA:

LENGTH/LONGITUD: $81.0 \mathrm{~km}$ (84.3 km cumulative)

AVERAGE STRIKE/RUMBO PROMEDIO: $-72.0^{\circ} \pm 10^{\circ}\left(\mathrm{N} 72.0^{\circ} \mathrm{W} \pm 10^{\circ}\right)$

AVERAGE DIP/INCLINACION PROMEDIO: $60^{\circ} \mathrm{S}$

SENSE OF MOVEMENT/SENTIDO DE MOVIMIENTO: Normal dextral

Comments/Comentarios: Presence of striae. They correspond to a NE-SW extension direction with $\sigma 3$ $\mathrm{N} 41^{\circ} \mathrm{E}$.

Presencia de estrias. Corresponden a una dirección de extensión NE-SW con $\sigma 3 \mathrm{~N} 41^{\circ} \mathrm{E}$.

GEOMORPHIC EXPRESSION/EXPRESION GEOMORFOLOGICA: Scarp of nearly $1000 \mathrm{~m}$ height. It limits the Sacaba basin to the north, and the scarp presents 6 ablation levels. In comparison, the southern border of the basin has only 3 erosional levels. This fault border probably presents reduced or no activity.

Escarpe de casi $1000 \mathrm{~m}$. Limita al norte la cuenca de Sacaba con presencia de 6 pisos de ablación a lo largo de relieve en vez de solamente 3 pisos de erosión en el borde sur de la cuenca, borde con activada reducida o sin actividad.

SLIP RATE/TASA DE MOVIMIENTO: Unknown/desconocida.

TIME OF MOST RECENT OF MOVEMENT/EDAD DEL ULTIMO MOVIMIENTO: Quaternary/Cuaternario $(<1.6 \mathrm{Ma})$

REFERENCES/REFERENCIAS

Lavenu, A., 1988, Etude tectonique et néotectonique de l'Altiplano et de la Cordillère orientale des Andes boliviennes: Travaux et Documents microfichés, v. 28, 420 pp., ORSTOM, Paris.

Lavenu, A., Mercier, J.L., 1991, Evolution du régime tectonique de l'Altiplano et de la Cordillère orientale des Andes de Bolivie du Miocène supérieur à l'Actuel: Géodynamique, v. 6, no. 1, p. 21-55.

\section{BO-12, TARIJA FAULT}

FAULT NUMBER/NUMERO DE LA FALLA: BO-12

FAULT NAME/NOMBRE DE LA FALLA: Tarija (informal name/sin nombre)

SYNOPSIS AND GEOLOGIC SETTING/SINOPSIS Y AMBIENTE GEOLOGICO: Quaternary activity described in 1988. General neotectonic field studies.

Actividad cuaternaria descrita en 1988. Estudios neotectónicos de campo generales. 
COMPILER, AFFILIATION, \& DATE OF COMPILATION/COMPILADOR, AFILIACION Y FECHA DE

COMPILACION: Alain Lavenu, IRD, France; 06-1996

TYPE OF STUDIES/TIPOS DE ESTUDIOS: Fieldwork and microstructural analysis.

Trabajos de campo y análisis microestructural.

FAULT GEOMETRY/GEOMETRIA DE LA FALLA:

LENGTH/LONGITUD: $54.4 \mathrm{~km}$ (56.1 km cumulative)

AVERAGE STRIKE/RUMBO PROMEDIO: $22.6^{\circ} \pm 22^{\circ}\left(\mathrm{N} 22.6^{\circ} \mathrm{E} \pm 22^{\circ}\right)$

AVERAGE DIP/INCLINACION PROMEDIO: $70^{\circ} \mathrm{W}$

SENSE OF MOVEMENT/SENTIDO DE MOVIMIENTO: Normal

Comments/Comentarios: Presence of striae. They correspond to a NNE-SSW extension direction with

$\sigma 3 \mathrm{~N} 201^{\circ} \mathrm{E}$. More than 100 measured faults affect the Pleistocene deposits. The numerical

microtectonic analysis, across all the basin, allows a specific "revolution" deformation, with $\sigma 3$ and $\sigma 2$

horizontal and very similar. It is difficult to distinguish the NNE-SSW extensional direction of $\sigma 3$ from the

ESE-WNW extensional direction of $\sigma 2$. These Pleistocene deposits are also affected by a E-W

compressional faulting deformation (strike-slip faults), with $\sigma 1 \mathrm{~N} 85^{\circ} \mathrm{E}$. It was not possible to distinguish the chronology between the extensional and compressional deformations. The N-S extensional state of stress observed in the Altiplano can be explained as the result of body force related to the effect of high compensated topography (Sébrier and others, 1985, 1988; Mercier and others, 1987, 1991; Froidevaux and Isacks, 1984) versus the E-W maximum horizontal stress due to the convergence. In the high Andes, the vertical stress increases with the weight of the cordillera and becomes $\sigma 1 ; \sigma 3$ is $\mathrm{N}$ $\mathrm{S}$. In the Subandean lowlands, tectonics is compressional [BO-14] and $\sigma 1$ is $\mathrm{E}-\mathrm{W}$. In zones such as Tarija, $1900 \mathrm{~m}$ in alt., the strike-slip deformation could correspond to an intermediate zone between subandean lowlands and high range. Nevertheless, it is dificult to explain the axial stress extension in a regional context (Lavenu and Mercier, 1991).

Presencia de estrias. Corresponden a una dirección de extensión NNE-SSW con $\sigma 3 \mathrm{~N} 201^{\circ} \mathrm{E}$. Más de 100 fallas normales medidas en la cuenca de Tarija afectan a los depositos pleistocenos. El análisis numérico de las fallas en toda la cuenca muestra una deformación particular, de revolución, con $\sigma 3$ y $\sigma 2$ horizontales y muy cercanos. Estos depositos se encuentran afectados también por una deformación en compresión de dirección E-W ( $\sigma 1 \mathrm{~N} 85^{\circ} \mathrm{E}$ ) (fallas de rumbo). No se pudo determinar la cronología entre tectónica extensiva y tectónica compresiva. El estado de esfuerzo en extensión de dirección N-S observado en el Altiplano se explica como el resultado de la fuerzas de volumen debidas a la alta topografía compensada (Sébrier and others, 1985, 1988; Mercier and others, 1987, 1992; Froidevaux and Isacks, 1984) versus el esfuerzo horizontal máximo $\approx \mathrm{E}-\mathrm{W}$ debido a la convergencia. En las zonas altas de los Andes, el esfuerzo vertical aumenta por el peso de la cordillera y se vuelve $\sigma 1 ; \sigma 3$ es N-S. En las zonas bajas, como la zona subandina por ejemplo, la tectónica es compresiva [BO-14] y $\sigma 1$ es EW. En zona como Tarija, $1900 \mathrm{~m}$ snm, la deformación en falla de rumbo podría corresponder a una zona intermedia entre tierras bajas subandinas y alta cordillera. Sin embargo queda dificil explicar y interpretar la extensión de revolución en un contexto regional (Lavenu and Mercier, 1991).

GEOMORPHIC EXPRESSION/EXPRESION GEOMORFOLOGICA: Eroded scarp/Escarpe erodado SLIP RATE/TASA DE MOVIMIENTO: Unknown/desconocida.

TIME OF MOST RECENT OF MOVEMENT/EDAD DEL ULTIMO MOVIMIENTO: Quaternary/Cuaternario $(<1.6 \mathrm{Ma})$

REFERENCES/REFERENCIAS

Froidevaux, C. and Isacks, B.L., 1984, The mechanical state of the Altiplano-Puna segment of the Andes: Earth Plenatry Science Letters, v. 71, p. 305-314.

Lavenu, A., 1988, Etude tectonique et néotectonique de l'Altiplano et de la Cordillère orientale des Andes boliviennes: Travaux et Documents microfichés, v. 28, 420 pp., ORSTOM, Paris.

Lavenu, A., Mercier, J.L., 1991, Evolution du régime tectonique de l'Altiplano et de la Cordillère orientale des Andes de Bolivie du Miocène supérieur à l'Actuel: Géodynamique, v. 6, no. 1, p. 21-55.

Mercier, J.L., Sorel, D., Simeakis, K., 1987, Changes in the state of stress in the overriding plate of a subduction zone-The Aegean arc from the Pliocene to the Present: Annales tectonicae, v. 1, no. 1, p. 20-39.

Mercier, J.L., Sébrier, M., Lavenu, A., Cabrera J., Bellier, O., Dumont, J.F., and Macharé, J., 1992, Changes in the tectonic regime above a subduction zone of Andean type-The Andes of Peru and Bolivia during the PliocenePleistocene: Journal of Geophysical Research, v. 97, no. B8, p. 11,945-11,982. 
Sébrier, M., Mercier, J.L., Macharé, J., Bonnot, D., Cabrera, J., and Blanc, J.L., 1988, The state of stress in an overriding plate situated above a flat slab-The Andes of Central Peru: Tectonics, v. 7, no. 4, p. 895-928.

Sébrier, M., Mercier, J.L., Mégard, F., Laubacher, G., and Carey-Gailhardis, E., 1985, Quaternary normal and reverse faulting and the state of stress in central Andes of Southern Peru: Tectonics, v. 4, no. 7, p. 739-780.

\section{BO-13, BENI RIVER FAULT}

FAULT NUMBER/NUMERO DE LA FALLA: BO-13

FAULT NAME/NOMBRE DE LA FALLA: Beni River (informal name)/ Rio Beni (sin nombre)

SYNOPSIS AND GEOLOGIC SETTING/SINOPSIS Y AMBIENTE GEOLOGICO: Quaternary activity described in 1996. General neotectonic field and fluvial traces studies.

Actividad cuaternaria descrita en 1996. Estudios neotectónicos de campo generales y de trazas de ríos.

COMPILER, AFFILIATION, \& DATE OF COMPILATION/COMPILADOR, AFILIACION Y FECHA DE COMPILACION: Alain Lavenu, IRD, France; 06-1996

TYPE OF STUDIES/TIPOS DE ESTUDIOS: Fieldwork/Trabajos de campo.

FAULT GEOMETRY/GEOMETRIA DE LA FALLA:

LENGTH/LONGITUD: $91.1 \mathrm{~km}$ (67.5 km cumulative)

AVERAGE STRIKE/RUMBO PROMEDIO: $4.5^{\circ} \pm 17^{\circ}\left(\mathrm{N} 4.5^{\circ} \mathrm{E} \pm 17^{\circ}\right)$

AVERAGE DIP/INCLINACION PROMEDIO: Unknown, eastward dip/Desconocido, hacia el este

SENSE OF MOVEMENT/SENTIDO DE MOVIMIENTO: Normal

GEOMORPHIC EXPRESSION/EXPRESION GEOMORFOLOGICA: Two N-S faults cut an upper terrace of the Beni River./Dos fallas N-S cortan una terraza alta del Río Beni.

SLIP RATE/TASA DE MOVIMIENTO: Unknown/desconocida.

TIME OF MOST RECENT OF MOVEMENT/EDAD DEL ULTIMO MOVIMIENTO: Quaternary/Cuaternario $(<1.6 \mathrm{Ma})$

REFERENCES/REFERENCIAS

Dumont, J.F., 1996, Neotectonics of the Subandes-Brazilian craton boundary using geomorphological data-The Marañon and Beni basins: Tectonophysics, v. 257, p. 137-151.

\section{BO-14, MANDEYAPECUA FAULT}

FAULT NUMBER/NUMERO DE LA FALLA: BO-14

FAULT NAME/NOMBRE DE LA FALLA: Mandeyapecua

SYNOPSIS AND GEOLOGIC SETTING/SINOPSIS Y AMBIENTE GEOLOGICO: Quaternary activity described in 1996. General tectonic field.

Actividad cuaternaria descrita en 1996. Estudios tectónicos de campo generales.

COMPILER, AFFILIATION, \& DATE OF COMPILATION/COMPILADOR, AFILIACION Y FECHA DE COMPILACION: Alain Lavenu, IRD, France; 06-1996

TYPE OF STUDIES/TIPOS DE ESTUDIOS: Fieldwork, seismic reflection.

Trabajos de campo, sísmica de reflexión.

FAULT GEOMETRY/GEOMETRIA DE LA FALLA:

LENGTH/LONGITUD: $486.6 \mathrm{~km}$ (507.8 km cumulative)

AVERAGE STRIKE/RUMBO PROMEDIO: $-6.7^{\circ} \pm 16^{\circ}\left(\mathrm{N} 6.7^{\circ} \mathrm{W} \pm 16^{\circ}\right)$

AVERAGE DIP/INCLINACION PROMEDIO: $60^{\circ} \mathrm{W}$, in cross-section $/ 60^{\circ} \mathrm{W}$ en corte.

SENSE OF MOVEMENT/SENTIDO DE MOVIMIENTO: Reverse/inversa

GEOMORPHIC EXPRESSION/EXPRESION GEOMORFOLOGICA: undescribed/no descrita

SLIP RATE/TASA DE MOVIMIENTO: Unknown/desconocida.

TIME OF MOST RECENT OF MOVEMENT/EDAD DEL ULTIMO MOVIMIENTO: Quaternary/Cuaternario $(<1.6 \mathrm{Ma})$

REFERENCES/REFERENCIAS

Marshall, L.G., and Sempéré, T., 1991, The Eocene to Pleistocene vertebrates of Bolivia and their stratigraphic context-A review, in Fósiles y Facies de Bolivia, 1: Revista Técnica de YPFB, p. 631-652.

Moretti, I., Baby, P., Mendez, E., and Zubieta, D., 1996, Hydrocarbon generation in relation to thrusting in the Sub Andean Zone from 18 to $22^{\circ} \mathrm{S}$ - Bolivia: Petroleum Geoscience, v. 2, p. 17-28. 
TABLE 1. QUATERNARY FAULTS OF BOLIVIA (FALLAS CUATERNARIAS DE BOLIVIA)

\begin{tabular}{|c|c|c|c|c|}
\hline $\begin{array}{l}\text { Number } \\
\text { Número }\end{array}$ & $\begin{array}{l}\text { Name of structure/ } \\
\text { Nombre de estructura }\end{array}$ & $\begin{array}{c}\text { Sense of } \\
\text { movement } \\
\text { Sentido de } \\
\text { movimiento }\end{array}$ & $\begin{array}{l}\text { Time of most } \\
\text { recent faulting } \\
\text { Edad del último } \\
\text { movimiento }\end{array}$ & $\begin{array}{c}\text { Slip rate }(\mathrm{mm} / \mathrm{yr}) \\
\text { Tasa de movimiento (mm/año) }\end{array}$ \\
\hline BO-01 & $\begin{array}{l}\text { Fault east of Achacachi/ } \\
\text { Falla este de Achacachi }\end{array}$ & Normal & $<1.6 \mathrm{Ma}$ & Unknown/desconocida \\
\hline BO-02 & $\begin{array}{l}\text { Fault east of Peñas/ } \\
\text { Falla este de Peñas }\end{array}$ & Normal & $<15 \mathrm{ka}$ & $\begin{array}{l}\text { Unknown, probably }<0.125 \text {; } \\
\text { desconocida, } \\
\text { probablemente }<0.125\end{array}$ \\
\hline BO-03 & $\begin{array}{l}\text { Kenko fault zone/ } \\
\text { Sistema de falla Kenko }\end{array}$ & Normal-sinistral & $<1.6 \mathrm{Ma}$ & $<0.2$ \\
\hline BO-04 & Llojeta fault/Falla de Llojeta & Normal-dextral & $<1.6 \mathrm{Ma}$ & $<0.2$ \\
\hline BO-05 & $\begin{array}{l}\text { Amachuma fault/ } \\
\text { Falla de Amachuma }\end{array}$ & Normal-sinistral & $<1.6 \mathrm{Ma}$ & Unknown/desconocida \\
\hline BO-06 & $\begin{array}{l}\text { Quebrada Minasa fault/ } \\
\text { Falla Quebrada Minasa }\end{array}$ & Normal-dextral & $<1.6 \mathrm{Ma}$ & $\begin{array}{l}\text { Unknown, probably }<0.2 ; \\
\text { desconocida, } \\
\text { probablemente }<0.2\end{array}$ \\
\hline BO-07 & Escoma fault/Falla Escoma & Normal & $<1.6 \mathrm{Ma}$ & Unknown/desconocida \\
\hline BO-08 & $\begin{array}{l}\text { Viacha fault zone/ Sistema de } \\
\text { falla de Viacha }\end{array}$ & Normal & $<1.6 \mathrm{Ma}$ & Unknown/desconocida \\
\hline BO-09 & $\begin{array}{l}\text { Ayo Ayo fault/Falla de Ayo } \\
\text { Ayo }\end{array}$ & Normal-dextral & $<1.6 \mathrm{Ma}$ & Unknown/desconocida \\
\hline BO-10 & $\begin{array}{l}\text { Cuenca de Charaña fault/ } \\
\text { Falla Cuenca de Charaña }\end{array}$ & Normal & $<1.6 \mathrm{Ma}$ & Unknown/desconocida \\
\hline BO-11 & Tunari fault/Falla Tunari & Normal-dextral & $<1.6 \mathrm{Ma}$ & Unknown/desconocida \\
\hline BO-12 & Tarija fault/Falla de Tarija & Normal & $<1.6 \mathrm{Ma}$ & Unknown/desconocida \\
\hline BO-13 & $\begin{array}{l}\text { Beni River fault/Falla del Río } \\
\text { Beni }\end{array}$ & Normal & $<1.6 \mathrm{Ma}$ & Unknown/desconocida \\
\hline BO-14 & $\begin{array}{l}\text { Mandeyapecua fault/ } \\
\text { Falla Mandeyapecua }\end{array}$ & Reverse & $<1.6 \mathrm{Ma}$ & Unknown/desconocida \\
\hline
\end{tabular}




\title{
FAULT DATABASE FOR CHILE \\ CH-01, ATACAMA FAULT ZONE
}

\author{
FAULT NUMBER/NUMERO DE LA FALLA: CH-01 \\ FAULT NAME/NOMBRE DE LA FALLA: Atacama (AFZ) \\ SYNOPSIS AND GEOLOGIC SETTING/SINOPSIS Y AMBIENTE GEOLOGICO:
}

The Atacama Fault Zone (AFZ) is a continuous fault zone extending over $1000 \mathrm{~km}$ in the Coastal Range of northern Chile, between Iquique $\left(20^{\circ} \mathrm{S}\right)$ and La Serena $\left(30^{\circ} \mathrm{S}\right)$. During the 60's and the $70^{\prime} \mathrm{s}$, authors considered that dextral motion along the AFZ occured during the early history of the fault (e.g., Saint Amand and Allen, 1960; Arabasz, 1971). More recent works agree in placing the origin of the AFZ in the Jurassic-early Cretaceous times showing ductile sinistral shear across the zone (Naranjo and others, 1984; Hervé, 1987; Scheuber and Andriessen, 1990). The existence of a sinistral ductile to brittle transtensional region would have been produced by the highly oblique convergence between the Phoenix and South American Plates and the AFZ (Scheuber and Reuter, 1992; Brown and others, 1993). Some authors proposed a predominantly vertical motion during late Cenozoic time (Arabasz, 1971; Okada, 1971). However, controversial interpretations also exist for this fault zone. According to Delouis and others (1998), "the formerly continuous ridge and valleys cut by the east-facing fault scarp are not horizontally offset, precluding any significant component of lateral displacement during the recent history of the fault". Conversely, Armijo and Thiele (1980) reported a predominant left-lateral component of motion along one of the main branches of the AFZ (e.g., Carmen Salar faults [CH-01c/d], northeast of Antofagasta), whereas Dewey and Lamb (1992) considered a small dextral component of displacement along the same section of the Carmen Salar fault. According to Delouis and others (1998), along the main branches of the AFZ, in the area of Salar del Carmen, Mejillones Peninsula and east and southeast of Antofagasta, no large scale horizontal block motions are taking place. Deformation is essentially characterized by vertical uplift, accompanied by horizontal extension produced by normal faulting.

La Zona de Falla de Atacama (ZFA) es una zona de falla contínua sobre más de $1000 \mathrm{~km}$, en la Cordillera de la Costa del norte de Chile, entre Iquique $\left(20^{\circ} \mathrm{S}\right)$ y La Serena $\left(30^{\circ} \mathrm{S}\right)$. Durante los años 60 y 70 , autores consideraron que un movimiento dextral a lo largo de la ZFA ocurrió a principio de la historia de la falla (Saint Amand and Allen, 1960; Arabasz, 1971). Trabajos más recientes logran situar el origen de la ZFA en el Jurásico-Cretacico inferior, mostrando zona de cizalle dúctil sinestral (Naranjo and others, 1984; Hervé, 1987; Scheuber y Andriessen, 1990). La existencia de una región en transtensión, sinestral dúctil a frágil, se debería a la alta oblicuidad de la convergencia entre las placas Phoenix, America del sur y la ZFA (Scheuber y Reuter, 1992; Brown and others, 1993). Otros autores proponen un movimiento essencialmente vertical durante el Cenozoico superior (Arabasz, 1971; Okada, 1971). Pero, interpretaciones diferentes existen a proposito de esta zona. Según Delouis and others (1998), "valles y crestas antiguamente continuos, cortados por el escarpe de falla con buzamiento al este, no son desplazados horizontalmente, excluyendo cualquier desplazamiento lateral con componente signicativo durante la historia reciente de la falla". Armijo y Thiele (1980) reportan un movimiento con componente lateral predominantemente sinestral a lo largo de una de las principales ramas de la ZFA (e.g., fallaa Salar del Carmen [CH-01c/d], nor-este de Antofagasta), mientras Dewey y Lamb (1992) consideraban una pequeña componente dextral a lo largo del mismo seccion de la Falla del Salar del Carmen. Según Delouis et al (1998), a lo largo de las ramas principales de la ZFA, en el area del Salar del Carmen, en la Peninsula de Mejillones y al este y sur-este de Antofagasta, no existen movimientos horizontales de bloques a gran escala. La deformación es esencialmente caracterizada por un levantamiento vertical, junto con una extensión horizontal producida por un fallamiento normal.

COMPILER, AFFILIATION, \& DATE OF COMPILATION/COMPILADOR, AFILIACION Y FECHA DE

COMPILACION: Alain Lavenu, IRD, France, and RicardoThiele, Univ. de Chile; Chile, 08-1997.

TYPE OF STUDIES/TIPOS DE ESTUDIOS: See discussion under separate fault sections/Ver discusión abajo en cada sección de falla.

FAULT GEOMETRY/GEOMETRIA DE LA FALLA:

LENGTH/LONGITUD: $508.3 \mathrm{~km}$ (858.3 km cumulative)

AVERAGE STRIKE/RUMBO PROMEDIO: $0.9^{\circ} \pm 23^{\circ}\left(\mathrm{N} 0.9^{\circ} \mathrm{E} \pm 23^{\circ}\right)$ 
AVERAGE DIP/INCLINACION PROMEDIO: Subvertical

SENSE OF MOVEMENT/SENTIDO DE MOVIMIENTO: Normal

NUMBER OF SECTIONS/NOMBRE DE LA SECCIONES: 5

Comments/Comentarios: For this compilation, five sections were defined for the Atacama fault zone.

Para esta compilación, cinco secciones se definieron para la Zona de Falla de Atacama.

\section{CH-01A, SALAR GRANDE FAULT, SECTION OF ATACAMA FAULT}

SECTION NUMBER/NUMERO DE LA SECCION: CH-01a

SECTION NAME/NOMBRE DE LA SECCION: Salar Grande fault (section of Atacama fault)/Falla Salar

Grande (seccion de falla de Atacama)

SYNOPSIS AND GEOLOGIC SETTING/SINOPSIS Y AMBIENTE GEOLOGICO: Quaternary activity.

General neotectonic field studies. Coastal Cordillera. This fault cuts sediment of the Salar Grande

(playa), probably Quaternary in age. This fault is compatible with a N-S right-lateral transpressional

tectonic regime, according to González and others (1997). This fault corresponds to the northern part of

the Salar Grande fault, more than $400 \mathrm{~km}$ long.

Actividad cuaternaria. Estudios neotectónicos de campo generales. Cordillera de la Costa. Corta

sedimentos del Salar Grande (playa) de edad cuaternaria. Esta falla es compatible con un régimen tectónico en transpresión dextral N-S según Gonzalez and others (1997). Esta falla corresponde a la parte norte de la falla del Salar Grande, de más de $400 \mathrm{~km}$ de largo.

TYPE OF STUDIES/TIPOS DE ESTUDIOS: Fieldwork and interpretation of satellite images.

Trabajos de campo y interpretación de imagenes satelital.

SECTION GEOMETRY/GEOMETRIA DE SECCION:

LENGTH/LONGITUD: $27.5 \mathrm{~km}$ (88.7 km cumulative)

AVERAGE STRIKE/RUMBO PROMEDIO: $-29.8^{\circ} \pm 19^{\circ}\left(\mathrm{N} 29.8^{\circ} \mathrm{W} \pm 19^{\circ}\right)$

AVERAGE DIP/INCLINACION PROMEDIO: Subvertical, towards East/subvertical, hacia el Este

SENSE OF MOVEMENT/SENTIDO DE MOVIMIENTO: Dextral-reverse/dextral-inversa

Comments/Comentarios: Fault more than $40 \mathrm{~km}$ long; average direction is NW-SE. Displacements yet to be quantified.

Falla de más de 40 kilómetros de largo, de dirección promedio NW-SE. Los desplazamientos no son todavia cuantificados.

GEOMORPHIC EXPRESSION/EXPRESION GEOMORFOLOGICA: This fault section has a 3-m-high scarp with uplift of the NW block.

Este seccion de la falla presenta un escarpe de 3 metros de altura con ascenso del bloque noroccidental.

SLIP RATE/TASA DE MOVIMIENTO: Unknown/desconocida

TIME OF MOST RECENT OF MOVEMENT/EDAD DEL ULTIMO MOVIMIENTO: Quaternary/Cuaternario $(<1.6$ $\mathrm{Ma})$

REFERENCES/REFERENCIAS

Gonzalez, G., Jensen, A., Schneider H., 1997, Deformaciones recientes en la terminación norte de la zona de Falla de Atacama; Salar Grande, norte de Chile-Partición de la deformación por efecto de bloqueamiento del oroclinal andino: VIII ${ }^{\circ}$ Cong. Geol. Chileno, I, 1, p. 80-84.

\section{CH-01B, PUNTA DE LOBOS FAULT, SECTION OF ATACAMA FAULT}

SECTION NUMBER/NUMERO DE LA SECCION: CH-01b

SECTION NAME/NOMBRE DE LA SECCION: Punta de Lobos fault (section of Atacama fault)/Falla de

Punta de Lobos (seccionde falla de Atacama)

SYNOPSIS AND GEOLOGIC SETTING/SINOPSIS Y AMBIENTE GEOLOGICO: General neotectonic field studies. Coastal Cordillera. Quaternary activity. This fault cuts Quaternary sediments. The direction of the fault is NW-SE, and it is compatible with a N-S right-lateral transpressional tectonic regime, according to González and others (1997). This fault corresponds to the northern part of the Salar Grande fault, more than $400 \mathrm{~km}$ long.

Actividad cuaternaria. Estudios neotectónicos de campo generales. Cordillera de la Costa. Corta sedimentos de edad cuaternaria. Esta falla, NW-SE, es compatible con un régimen tectónico en 
transpresión dextral N-S según Gonzalez and others (1997). Esta falla corresponde a la parte norte de la falla del Salar Grande, de más de $400 \mathrm{~km}$ de largo.

TYPE OF STUDIES/TIPOS DE ESTUDIOS: Fieldwork and interpretation of satellite images.

Trabajos de campo y interpretación de imagenes satelital.

SECTION GEOMETRY/GEOMETRIA DE SECCION:

LENGTH/LONGITUD: $41.8 \mathrm{~km}$ (41.9 km cumulative)

AVERAGE STRIKE/RUMBO PROMEDIO: $-38.4^{\circ} \pm 12^{\circ}\left(\mathrm{N} 38.4^{\circ} \mathrm{W} \pm 12^{\circ}\right)$

AVERAGE DIP/INCLINACION PROMEDIO: Subvertical, toward the East/Subvertical, hacia el E

SENSE OF MOVEMENT/SENTIDO DE MOVIMIENTO: Dextral-reverse/Dextral-inverso

Comments/Comentarios: Fault more than $25 \mathrm{~km}$ long, trending N-S.

Falla de más de $25 \mathrm{~km}$ de largo, de dirección promedio N-S.

GEOMORPHIC EXPRESSION/EXPRESION GEOMORFOLOGICA: Streams subperpendicular to the fault zone are displaced between 3 and $5 \mathrm{~m}$ in a right lateral sense.

Drenajes subperpendiculares a la zona de falla se encuentran desplazados entre 3 y 5 metros en un sentido dextral.

SLIP RATE/TASA DE MOVIMIENTO: Unknown/desconocida

TIME OF MOST RECENT OF MOVEMENT/EDAD DEL ULTIMO MOVIMIENTO: Quaternary/Cuaternario $(<1.6 \mathrm{Ma})$

REFERENCES/REFERENCIAS

Gonzalez, G., Jensen, A., Schneider H., 1997, Deformaciones recientes en la terminación norte de la zona de Falla de Atacama; Salar Grande, norte de Chile-Partición de la deformación por efecto de bloqueamiento del oroclinal andino: VIII ${ }^{\circ}$ Cong. Geol. Chileno, I, 1, p. 80-84.

\section{CH-01C, NORTHERN PART OF CARMEN SALAR FAULT, SECTION OF ATACAMA FAULT}

SECTION NUMBER/NUMERO DE LA SECCION: CH-01C

SECTION NAME/NOMBRE DE LA SECCION: Northern part of Carmen Salar fault (section of Atacama fault)/Parte norte de la Falla del Salar del Carmen (seccion de falla de Atacama)

SYNOPSIS AND GEOLOGIC SETTING/SINOPSIS Y AMBIENTE GEOLOGICO: Quaternary activity.

General neotectonic field studies. Coastal Cordillera. This fault cuts sediment of probable Quaternary

age. The fault is compatible with a tectonic regime of $\mathrm{N}-\mathrm{S}$ right-lateral transpression, according to Gonzalez and others (1997).

Actividad cuaternaria. Estudios neotectónicos de campo generales. Cordillera de la Costa. Corta sedimentos de edad cuaternaria. Esta falla es compatible con un régimen tectónico en transpresión dextral N-S según Gonzalez and others (1997).

COMPILER, AFFILIATION, \& DATE OF COMPILATION/COMPILADOR, AFILIACION Y FECHA DE

COMPILACION: Alain Lavenu, IRD, France, and RicardoThiele, Univ. de Chile; Chile, 08-1997.

TYPE OF STUDIES/TIPOS DE ESTUDIOS: Fieldwork and interpretation of satellite images.

Trabajos de campo y interpretación de imagenes satelital.

SECTION GEOMETRY/GEOMETRIA DE SECCION:

LENGTH/LONGITUD: 230.9 km (321.6 km cumulative)

AVERAGE STRIKE/RUMBO PROMEDIO: $12.5^{\circ} \pm 18^{\circ}\left(\mathrm{N} 12.5^{\circ} \mathrm{E} \pm 18^{\circ}\right)$

AVERAGE DIP/INCLINACION PROMEDIO: $70-80^{\circ} E$

SENSE OF MOVEMENT/SENTIDO DE MOVIMIENTO: Dextral-reverse/Dextral-inverso

Comments/Comentarios: This fault is more than $50 \mathrm{~km}$ long with $\mathrm{N}-\mathrm{S}$ average direction. Displacements have not been quantified yet.

Falla de más de $50 \mathrm{~km}$ de largo con dirección promedio N-S. Los desplazamientos no son todavia cuantificados.

GEOMORPHIC EXPRESSION/EXPRESION GEOMORFOLOGICA: This fault section has a 5-m high scarp with uplift of the eastern block.

Este seccion de la falla presenta un escarpe de 5 metros de altura con ascenso del bloque oriental. SLIP RATE/TASA DE MOVIMIENTO: Unknown/desconocida 
TIME OF MOST RECENT OF MOVEMENT/EDAD DEL ULTIMO MOVIMIENTO: Quaternary/Cuaternario $(<1.6 \mathrm{Ma})$

REFERENCES/REFERENCIAS

Gonzalez, G., Jensen, A., Schneider H., 1997, Deformaciones recientes en la terminación norte de la zona de Falla de Atacama; Salar Grande, norte de Chile-Partición de la deformación por efecto de bloqueamiento del oroclinal andino: VIII ${ }^{\circ}$ Cong. Geol. Chileno, I, 1, p. 80-84.

\section{CH-01D, SOUTHERN PART OF CARMEN SALAR FAULT, SECTION OF ATACAMA FAULT}

SECTION NUMBER/NUMERO DE LA SECCION: CH-01d

SECTION NAME/NOMBRE DE LA SECCION: Southern part of Carmen Salar fault (section of Atacama

fault)/Parte sur de la falla de Salar deCarmen (seccion de falla de Atacama)

SYNOPSIS AND GEOLOGIC SETTING/SINOPSIS Y AMBIENTE GEOLOGICO: Very recent activity (late

Quaternary). New ruptures formed during the July 1995 earthquake (Monfret and others, 1995) in

Estación Uribe, Salar del Carmen. General neotectonic field studies. Coastal Cordillera. This fault cuts sediment of probable Neogene to Quaternary age. The large (composite) scarp faces eastward and is between 200 and $400 \mathrm{~m}$ high. Young scarps represent 8 to $10 \mathrm{~m}$ of throw. Fresh ruptures form a $70 \mathrm{~cm}$ scarp. There is an antithetic fault strand with dip towards the W. Compatible with an E-W extensional tectonic regime.

Actividad muy reciente (Cuaternario inferior). Rupturas nuevas debidas al sismo de julio 1995, en

Estación Uribe, Salar del Carmen. Estudios neotectónicos de campo generales. Cordillera de la Costa. Corta sedimentos de edad probable neógena a cuaternaria. El escarpe resultante, con buzamiento al este, tiene entre 200 y $400 \mathrm{~m}$. Escarpes frescos presentan rechazo de 8 a $10 \mathrm{~m}$. Las rupturas recientes presentan un escarpe de $70 \mathrm{~cm}$. Presencia de falla antitéticas con buzamiento al W. Esta falla es compatible con un régimen tectónico en extensión E-W.

TYPE OF STUDIES/TIPOS DE ESTUDIOS: Fieldwork and interpretation of aerial photographs.

Trabajos de campo y fotointerpretación.

SECTION GEOMETRY/GEOMETRIA DE SECCION:

LENGTH/LONGITUD: $100.5 \mathrm{~km}$ (102.1 km cumulative)

AVERAGE STRIKE/RUMBO PROMEDIO: $10.3^{\circ} \pm 16^{\circ}\left(\mathrm{N} 10.3^{\circ} \mathrm{E} \pm 16^{\circ}\right)$

AVERAGE DIP/INCLINACION PROMEDIO: East, dip angle unknown/Este, inclinación del plano de falla desconocida.

SENSE OF MOVEMENT/SENTIDO DE MOVIMIENTO: Normal

Comments/Comentarios: This fault section is more than $60 \mathrm{~km}$ long, trending N20-30 $\mathrm{E}$; no notable

lateral displacement. Delouis and others (1998) pointed out the appearance of normal faulting.

Nevertheless, Armijo and Thiele (1990) indicate a left-lateral 100-m displacement component, that was not confirmed by Delouis and others.

Este seccion de falla de más de $60 \mathrm{~km}$ de largo, de dirección promedio N20-30E sin desplazamientos laterales notables. Delouis and others (1998) señalan la aparencia de un fallamiento normal. Sin embargo, Armijo y Thiele (1990) notaban un desplazamiento de 100 metros en componente sinestral, que no fue confirmado por Delouis and others

GEOMORPHIC EXPRESSION/EXPRESION GEOMORFOLOGICA: Cumulative scarp is 200-400 m high.

Fresh scarps with throw of between 8 and $10 \mathrm{~m}$. Recent ruptures form $70 \mathrm{~cm}$ high scarps. Historic ruptures (from 1995) are a few cm high.

Escarpe acumulativo de 200 a $400 \mathrm{~m}$. Escarpes frescos con rechazo de 8 a $10 \mathrm{~m}$. Rupturas historicas (o de 1995) de algunos cm de alto.

RECURRENCE INTERVAL/INTERVALO DE RECURRENCIA: Unknown/desconocido

Comments/Comentarios: Ruptures look very young.

Las rupturas presentan un aspecto muy joven.

SLIP RATE/TASA DE MOVIMIENTO: Unknown/desconocida

TIME OF MOST RECENT OF MOVEMENT/EDAD DEL ULTIMO MOVIMIENTO: Historic (1995)/Histórico (1995)

Comments/Comentarios: New ruptures formed during the July 1995 earthquake in Estación Uribe, 
Salar del Carmen. Other fresh scarps suggest post glacial or Holocene movement ( $<15 \mathrm{ka})$.

Rupturas nuevas debidas al sismo de julio 1995, en Estación Uribe, Salar del Carmen. Otro escarpes frescos indican movimiento post glacial o Holoceno $(<15 \mathrm{ka})$.

REFERENCES/REFERENCIAS

Armijo, R., and Thiele, R., 1990, Active faulting in Northern Chile-Ramp stacking and lateral decoupling along a subduction plate boundary?: Earth and Planetary Science Letters, v. 98, p. 40-61.

Delouis B., 1996, Subduction et déformation continentale au Nord-Chili: France, Univ. Louis Pasteur, Thèse de Doctorat, $262 \mathrm{p}$.

Delouis B., Philip H., Dorbath L., and Cisternas A., 1998, Recent crustal deformation in the Antofagasta region (Northern Chile) and the subduction process: Geophysical Journal International, v. 132, p. 302-338.

Monfret T., Dorbath L., Caminade, J.P., Pardo, M., Comte, D., Ponce, L. , Cisternas, A., Delouis, B., Rivera, L., 1995, The July 30, 1995 Antofagasta earthquake-A "hypocritical" seismic event: EOS, Abstracts of the Fall Meeting of the Amer. Geophys. Union, v. 76, no. 46, p. F427.

\section{CH-01E, SIERRA DE REMIENDOS FAULT, SECTION OF ATACAMA FAULT}

SECTION NUMBER/NUMERO DE LA SECCION: CH-01e

SECTION NAME/NOMBRE DE LA SECCION: Sierra de Remiendos fault (section of Atacama fault)/Falla

Sierra de Remiendos (seccion de falla de Atacama)

SYNOPSIS AND GEOLOGIC SETTING/SINOPSIS Y AMBIENTE GEOLOGICO: General neotectonic field studies. Coastal Cordillera. Very recent activity (Quaternary). Both old and new ruptures exist, the latter due to the July 30, 1995 Antofagasta earthquake (Monfret and others, 1995), between Paposo and Antofagasta. According to Delouis and others (1997), the most recent ruptures formed a $20-\mathrm{cm}$ high vertical scarp. This fault is compatible with an E-W extensional tectonic regime.

Estudios neotectónicos de campo generales. Cordillera de la Costa. Actividad (Cuaternaria) muy reciente. Existen rupturas antiguea y rupturas nuevas debidas al Sierra de Remiendos sismo del 30 de julio de 1995, entre Paposo y Antofagasta según Delouis and others (1997). La falla corta sedimentos de edad probable neógena a cuaternaria. Las rupturas más recientes presentan un escarpe vertical de $20 \mathrm{~cm}$. Esta falla es compatible con un régimen tectónico en extensión E-W.

TYPE OF STUDIES/TIPOS DE ESTUDIOS: Fieldwork and interpretation of aerial photographs.

Trabajos de campo y fotointerpretación.

SECTION GEOMETRY/GEOMETRIA DE SECCION:

LENGTH/LONGITUD: $157.9 \mathrm{~km}$ (304.0 km cumulative)

AVERAGE STRIKE/RUMBO PROMEDIO: $-0.1^{\circ} \pm 14^{\circ}\left(\mathrm{N} 0.1^{\circ} \mathrm{W} \pm 14^{\circ}\right)$

AVERAGE DIP/INCLINACION PROMEDIO: Dip subvertical to east/Buzamiento subvertical a este

SENSE OF MOVEMENT/SENTIDO DE MOVIMIENTO: Normal

GEOMORPHIC EXPRESSION/EXPRESION GEOMORFOLOGICA: Vertical scarp $20 \mathrm{~cm}$ high.

Escarpe vertical de $20 \mathrm{~cm}$.

RECURRENCE INTERVAL/INTERVALO DE RECURRENCIA: Unknown/desconocida

Comments/Comentarios: The rupture can be followed for several hundred meters. Recent ruptures form a vertical scarp $20 \mathrm{~cm}$ high.

La ruptura se sigue sobre varios centenares de metros. Las rupturas las más recientes presentan un escarpe vertical de $20 \mathrm{~cm}$.

SLIP RATE/TASA DE MOVIMIENTO: Unknown/desconocida

TIME OF MOST RECENT OF MOVEMENT/EDAD DEL ULTIMO MOVIMIENTO: Historic/Historico (1995)

Comments/Comentarios: Ruptures from the July 30, 1995 Sierra de Remiendos earthquake, between Paposo and Antofagasta according to Delouis and others (1997). Other fresh scarps suggest post glacial or Holocene movement ( $<15 \mathrm{ka})$.

Exiten rupturas nuevas debidas al sismo de Antofagasta del 30 de julio de 1995, entre Paposo y

Antofagasta según Delouis and others (1997). Otro escarpes frescos indican movimiento post glacial o Holoceno $(<15 \mathrm{ka})$.

NAME OF EARTHQUAKE/NOMBRE DEL TERREMOTO: Antofagasta

DATE/FECHA MAGNITUDE OR INTENSITY/MAGNITUD O INTENSIDAD: July 30,1995, $\mathrm{M}_{\mathrm{s}} 7.3$

MOMENT MAGNITUDE $M_{w} 8.0$ 
LENGTH OF SURFACE RUPTURE/LONGITUD DE RUPTURA: The rupture can be followed for several hundred meters./La ruptura se sigue sobre varios centenares de metros.

MAXIMUM SLIP AT SURFACE/DESPLAZAMIENTO MAXIMO EN SUPERFICIE: Vertical displacement of $20 \mathrm{~cm}$./Desplazamiento vertical de $20 \mathrm{~cm}$.

SENSE OF MOVEMENT/SENTIDO DE MOVIMIENTO: Normal fault, vertical movement./Falla normal, movimiento vertical.

REFERENCES/REFERENCIAS

Delouis, B., Monfret, T., Dorbath, L., Pardo, M., Rivera, L., Comte, D., Haessler, H., Caminade, J.P., Ponce, L., Kausel, E., Cisternas, A., 1997, The $M_{w}=8.0$ Antofagasta (Northern Chile) earthquake of July 30, 1995-A precursor to the end of the large 1877 gap: Bull. Seis. Soc. Amer., v. 87, no. 2, p. 427-445.

Monfret T., Dorbath L., Caminade, J.P., Pardo, M., Comte, D., Ponce, L. , Cisternas, A., Delouis, B., Rivera, L., 1995, The July 30, 1995 Antofagasta earthquake-A hypocritical seismic event: EOS, Abstracts of the Fall Meeting of the Amer. Geophys. Union, v. 76, no. 46, p. F427.

\section{CH-02, MORRO MEJILLONES FAULT}

FAULT NUMBER/NUMERO DE LA FALLA: CH-02

FAULT NAME/NOMBRE DE LA FALLA: Morro Mejillones

SYNOPSIS AND GEOLOGIC SETTING/SINOPSIS Y AMBIENTE GEOLOGICO: Very recent activity

(Quaternary). General neotectonic field studies. Coastal Cordillera. This fault cuts sediment of probable

Neogene to Quaternary age. The cumulative scarp faces eastward, and it is as much as $400-500 \mathrm{~m}$ high; it shows a complex pattern of faceted spurs and uplifted wave-cut abrasion surfaces. The most recent ruptures from scarps are between $30 \mathrm{~cm}$ and $1.5 \mathrm{~m}$ high. This fault is compatible with an E-W extensional tectonic regime.

Actividad (Cuaternaria) muy reciente. Estudios neotectónicos de campo generales. Cordillera de la Costa. La falla corta sedimentos de edad probable neógena a cuaternaria. El escarpe total, con buzamiento al este, tiene hasta 400-500 m con un conjunto complejo de facetas triangulares y superficies de abrasion marinas levantadas. Las rupturas más recientes presentan un escarpe de $30 \mathrm{~cm}$ a $1.5 \mathrm{~m}$. Esta falla es compatible con un régimen tectónico en extensión E-W.

COMPILER, AFFILIATION, \& DATE OF COMPILATION/COMPILADOR, AFILIACION Y FECHA DE COMPILACION: Alain Lavenu, IRD, France, and RicardoThiele, Univ. de Chile; Chile, 08-1997.

TYPE OF STUDIES/TIPOS DE ESTUDIOS: Fieldwork and interpretation of aerial photographs.

Trabajos de campo y fotointerpretación.

FAULT GEOMETRY/GEOMETRIA DE LA FALLA:

LENGTH/LONGITUD: $25.0 \mathrm{~km}$ (25.4 km cumulative)

AVERAGE STRIKE/RUMBO PROMEDIO: $4.6^{\circ} \pm 11^{\circ}\left(\mathrm{N} 4.6^{\circ} \mathrm{E} \pm 11^{\circ}\right)$

AVERAGE DIP/INCLINACION PROMEDIO: Unknown, eastward dip/Desconocida, hacia el este SENSE OF MOVEMENT/SENTIDO DE MOVIMIENTO: Normal

Comments/Comentarios: No evidence of lateral movement. Purely normal movement proved by the existence of tension gashes parallel to the fault.

No hay evidencias de movimiento lateral. Movimiento puramente normal comprobado por la presensia de tension gashes paralelas a la falla.

GEOMORPHIC EXPRESSION/EXPRESION GEOMORFOLOGICA: The main fault scarp is between 8 and $10 \mathrm{~m}$ high on old alluvial fans and is between 5 and $6 \mathrm{~m}$ on intermediate age fans. The most recent fans do not have scarps.

El escarpe de la falla principal tiene 8 a $10 \mathrm{~m}$ de alto en los abanicos aluviales antiguos y 5 a $6 \mathrm{~m}$ en los abanicos aluviales de edad intermedia. Los abanicos más recientes no presentan escarpes.

COMMENTS/COMENTARIOS: An open gash on the trace of the main fault in the most recent alluvial fan may be the manifestation of a recent seismic (shaking) event.

Una grieta abierta en la traza de la falla principal en uno de los abanicos alluviales más recientes podría ser la manifestación de un evento sísmico reciente.

SLIP RATE/TASA DE MOVIMIENTO: Unknown/desconocida

TIME OF MOST RECENT OF MOVEMENT/EDAD DEL ULTIMO MOVIMIENTO: Quaternary/Cuaternario $(<1.6 \mathrm{Ma})$ 


\section{REFERENCES/REFERENCIAS}

Armijo, R., Thiele, R., 1990, Active faulting in northern Chile: Ramp stacking and lateral decoupling along a subduction plate boundary? Earth and Planetary Science Letters, v. 98, p. 40-61.

Delouis, B., 1996, Subduction et déformation continentale au Nord-Chili: France, Ph. D.,Univ. Louis Pasteur, 262 p.

Delouis, B., Philip, H., Dorbath, L., Cisternas, A., 1998, Recent crustal deformation in the Antofagasta region (Northern Chile) and the subduction process: Geophysical Journal International, 132, p. 302-338.

Ortlieb, L., Goy, J.L., Zazo, C., Hillaire-Marcel, C., Ghaleb, B., Guzman, N., Thiele, R., 1996, Quaternary morphostratigraphy and vertical deformation in Mejillones Peninsula, Northern Chile: St. Malo, France, Third ISAG, p. 211-214.

\section{CH-03, CERRO GORDO FAULT, ATACAMA FAULT ZONE}

\section{FAULT NUMBER/NUMERO DE LA FALLA: CH-03}

FAULT NAME/NOMBRE DE LA FALLA: Cerro Gordo (Atacama fault zone/sistema falla de Atacama) SYNOPSIS AND GEOLOGIC SETTING/SINOPSIS Y AMBIENTE GEOLOGICO: Very recent activity

(Quaternary). General neotectonic field studies. Coastal Cordillera. This fault cuts sediment of probable Neogene to Quaternary age. Scarp of nearly $2 \mathrm{~m}$ height. The average direction of this fault is $\mathrm{N} 25^{\circ} \mathrm{E}$, and it is compatible with an E-W extensional tectonic regime.

Actividad (Cuaternaria) muy reciente. Estudios neotectónicos de campo generales. Cordillera de la Costa. Corta sedimentos de edad probable neógena a cuaternaria. Escarpe de $2 \mathrm{~m}$. Esta falle de dirección promedio $\mathrm{N} 25^{\circ} \mathrm{E}$ es compatible con un régimen tectónico en extension E-W.

COMPILER, AFFILIATION, \& DATE OF COMPILATION/COMPILADOR, AFILIACION Y FECHA DE COMPILACION: Alain Lavenu, IRD, France, and RicardoThiele, Univ. de Chile; Chile, 08-1997.

TYPE OF STUDIES/TIPOS DE ESTUDIOS: Fieldwork and interpretation of aerial photographs

Trabajos de campo y fotointerpretación.

FAULT GEOMETRY/GEOMETRIA DE LA FALLA:

LENGTH/LONGITUD: $18.5 \mathrm{~km}$ (18.5 km cumulative)

AVERAGE STRIKE/RUMBO PROMEDIO: $15.7^{\circ} \pm 7^{\circ}\left(\mathrm{N} 15.7^{\circ} \mathrm{E} \pm 7^{\circ}\right)$

AVERAGE DIP/INCLINACION PROMEDIO: East; dip angle unknown/Este; buzamiento de falla desconocida.

SENSE OF MOVEMENT/SENTIDO DE MOVIMIENTO: Normal-sinistral.

Comments/Comentarios: Fault has $3 \mathrm{~m}$ left-lateral and $2 \mathrm{~m}$ vertical displacement.

Desplazamiento de $3 \mathrm{~m}$ en sentido sinestral y $2 \mathrm{~m}$ en sentido vertical.

GEOMORPHIC EXPRESSION/EXPRESION GEOMORFOLOGICA: The scarp is as much as $2 \mathrm{~m}$ high.

Sediment is trapped in small playa lakes along the fault.

El escarpe puede alcanzar $2 \mathrm{~m}$ de alto. Sedimentos se entrampan en pequeños salares a lo largo de la falla.

RECURRENCE INTERVAL/INTERVALO DE RECURRENCIA: Unknown/desconocido

SLIP RATE/TASA DE MOVIMIENTO: Unknown/desconocida

TIME OF MOST RECENT OF MOVEMENT/EDAD DEL ULTIMO MOVIMIENTO: Quaternary/Cuaternario $(<1.6 \mathrm{Ma})$

Comments/Comentarios: The activity could be more recent, possibly post glacial ( $<15 \mathrm{ka})$

El movimiento podría ser mas recientes, de edad post-glacial ( $<15 \mathrm{ka})$.

REFERENCES/REFERENCIAS

Delouis B., 1996, Subduction et déformation continentale au Nord-Chili: France, Ph. D., Univ. Louis Pasteur, 262 p.

Delouis B., Philip H., Dorbath L., and Cisternas A., 1998, Recent crustal deformation in the Antofagasta region

(Northern Chile) and the subduction process: Geophysical Journal International, v. 132, p. 302-338.

\section{CH-04, CERRO MORENO FAULT, ATACAMA FAULT ZONE}

\section{FAULT NUMBER/NUMERO DE LA FALLA: $\mathrm{CH}-04$}

FAULT NAME/NOMBRE DE LA FALLA: Cerro Moreno (Atacama fault zone/sistema falla de Atacama) SYNOPSIS AND GEOLOGIC SETTING/SINOPSIS Y AMBIENTE GEOLOGICO: Very recent activity

(Quaternary). General neotectonic field studies. Coastal Cordillera. This fault cuts sediment of probable Neogene to Quaternary age. The scarps face eastward and are as much as $60 \mathrm{~cm}$ high, with a $5 \mathrm{~m}$ left- 
lateral displacement according to Armijo and Thiele, 1990 (primarily a left-lateral fault with small normal component). The ratio between horizontal and vertical displacement increases in a NE direction. The fault has an average trend of $\mathrm{N} 25^{\circ} \mathrm{E}$, and is compatible with an $\mathrm{E}-\mathrm{W}$ extensional tectonic regime (Delouis and others, 1998).

Actividad (Cuaternaria) muy reciente. Estudios neotectónicos de campo generales. Cordillera de la Costa.Corta sedimentos de edad probable neógena a cuaternaria. El escarpe, con buzamiento al este, tiene hasta $60 \mathrm{~cm}$ con un desplazamiento lateral sinestral de $5 \mathrm{~m}$ según Armijo y Thiele 1990 (falla sinestral con componente normal debil). La relación desplazamiento horizontal vs desplazamiento vertical aumenta cuando la falla tiene una dirección hacia el NE. Esta falla de dirección promedio $\mathrm{N} 25^{\circ} \mathrm{E}$ es compatible con un regimén tectónico en extensión E-W (Delouis and others, 1998).

COMPILER, AFFILIATION, \& DATE OF COMPILATION/COMPILADOR, AFILIACION Y FECHA DE COMPILACION: Alain Lavenu, IRD, France, and RicardoThiele, Univ. de Chile; Chile, 08-1997.

TYPE OF STUDIES/TIPOS DE ESTUDIOS: Fieldwork and interpretation of aerial photographs.

Trabajos de campo y fotointerpretación.

FAULT GEOMETRY/GEOMETRIA DE LA FALLA:

LENGTH/LONGITUD: $47.4 \mathrm{~km}$ (45.6 km cumulative)

AVERAGE STRIKE/RUMBO PROMEDIO: $14.8^{\circ} \pm 9^{\circ}\left(\mathrm{N} 14.8^{\circ} \mathrm{E} \pm 9^{\circ}\right)$

AVERAGE DIP/INCLINACION PROMEDIO: East; dip angle unknown/Este; buzamiento del plano de falla desconocida

SENSE OF MOVEMENT/SENTIDO DE MOVIMIENTO: Sinistral-normal

GEOMORPHIC EXPRESSION/EXPRESION GEOMORFOLOGICA: According to Delouis and others

(1998), this scarp is $1.8 \mathrm{~m}$ high./ Según Delouis and others (1998), este escarpe tiene $1.8 \mathrm{~m}$ de alto.

RECURRENCE INTERVAL/INTERVALO DE RECURRENCIA: Unknown/desconocido

SLIP RATE/TASA DE MOVIMIENTO: Unknown/desconocida

TIME OF MOST RECENT OF MOVEMENT/EDAD DEL ULTIMO MOVIMIENTO: Quaternary/Cuaternario $(<1.6 \mathrm{Ma})$

REFERENCES/REFERENCIAS

Armijo, R., Thiele, R., 1990, Active faulting in northern Chile: ramp stacking and lateral decoupling along a subduction plate boundary? Earth and Planetary Science Letters, v. 98, p. 40-61.

Delouis, B., 1996, Subduction et déformation continentale au Nord-Chili: France, Univ. Louis Pasteur, Thèse de Doctorat, $262 \mathrm{p}$.

Delouis, B., Philip, H., Dorbath, L., Cisternas, A., 1998, Recent crustal deformation in the Antofagasta region (Northern Chile) and the subduction process: Geophysical Journal International, 132, p. 302-338.

\section{CH-05, CERRO FORTUNA FAULT}

\section{FAULT NUMBER/NUMERO DE LA FALLA: CH-05}

FAULT NAME/NOMBRE DE LA FALLA: Cerro Fortuna (Atacama fault zone/sistema falla de Atacama) SYNOPSIS AND GEOLOGIC SETTING/SINOPSIS Y AMBIENTE GEOLOGICO: Very recent activity

(Quaternary). General neotectonic field studies. Limits several westward tilted blocks, $1 \mathrm{~km}$ wide and 6

$\mathrm{km}$ long in the northernmost part of the Coastal Cordillera. This fault cuts sediments of probable Neogene to Quaternary age. The cumulative scarp, dipping eastward, is as much as $60 \mathrm{~m}$ high. The most recent faults form scarps ranging from $30 \mathrm{~cm}$ to $1.5 \mathrm{~m}$ in height. This fault is compatible with an E-W extensional tectonic regime.

Actividad (Cuaternaria) muy reciente. Estudios neotectónicos de campo generales. Limita varios bloques basculados hacia el oeste de $1 \mathrm{~km}$ de ancho con $6 \mathrm{~km}$ de largo en el parte norte de la Cordillera de la Costa. Corta sedimentos de edad probable neógena a cuaternaria. El escarpe total, con buzamiento al este, tiene hasta $60 \mathrm{~m}$. Las rupturas más recientes presentan un escarpe de $30 \mathrm{~cm}$ a 1.5 $\mathrm{m}$. Esta falla es compatible con un régimen tectónico en extensión E-W.

COMPILER, AFFILIATION, \& DATE OF COMPILATION/COMPILADOR, AFILIACION Y FECHA DE COMPILACION: Alain Lavenu, IRD, France, and RicardoThiele, Univ. de Chile; Chile, 08-1997.

TYPE OF STUDIES/TIPOS DE ESTUDIOS: Fieldwork and interpretation of aerial photographs.

Trabajos de campo y fotointerpretación. 
FAULT GEOMETRY/GEOMETRIA DE LA FALLA:

LENGTH/LONGITUD: $94.7 \mathrm{~km}$ (115.0 km cumulative)

AVERAGE STRIKE/RUMBO PROMEDIO: $11.1^{\circ} \pm 16^{\circ}\left(\mathrm{N} 11.1^{\circ} \mathrm{E} \pm 16^{\circ}\right)$

AVERAGE DIP/INCLINACION PROMEDIO: $60^{\circ} \mathrm{E}$

SENSE OF MOVEMENT/SENTIDO DE MOVIMIENTO: Normal

Comments/Comentarios: This fault is more than $80 \mathrm{~km}$ long, trending N5-15² E. Armijo and Thiele (1990) observed a left-lateral component of movement.

Falla de más de $80 \mathrm{~km}$ de largo, de dirección promedio N5-15E. Armijo y Thiele (1990) observaron una componente sinestral.

GEOMORPHIC EXPRESSION/EXPRESION GEOMORFOLOGICA: Forms 60-m high cumulative scarp,

fresh scarps from $30 \mathrm{~cm}$ to $1.5 \mathrm{~m}$ high. Tension gashes appear parallel to the scarp.

Escarpe total de $60 \mathrm{~m}$, con escarpe frescos de $30 \mathrm{~cm}$ a $1.5 \mathrm{~m}$. Existencia de grietas de tensión paralelas al escarpe.

RECURRENCE INTERVAL/INTERVALO DE RECURRENCIA: Unknown/desconocida

Comments/Comentarios: The fault remained inactive for a long period, but the presence of a fresh rupture on an old eroded scarp indicates that it has been reactivated two times recently.

La falla quedó inactiva durante un largo periodo, pero la presencia de una ruptura fresca y de un antiguo escarpe erodado indica que fue reactivada dos veces recientemente.

SLIP RATE/TASA DE MOVIMIENTO: Unknown/desconocida

TIME OF MOST RECENT OF MOVEMENT/EDAD DEL ULTIMO MOVIMIENTO: Quaternary/Cuaternario $(<1.6 \mathrm{Ma})$

REFERENCES/REFERENCIAS

Armijo, R., Thiele, R., 1990, Active faulting in northern Chile: ramp stacking and lateral decoupling along a subduction plate boundary?: Earth and Planetary Science Letters, v. 98, p. 40-61.

Delouis, B., 1996, Subduction et déformation continentale au Nord-Chili: France, Ph.D., Univ. Louis Pasteur, 262 p.

Delouis, B., Philip, H., Dorbath, L., Cisternas, A., 1998, Recent crustal deformation in the Antofagasta region (Northern Chile) and the subduction process: Geophysical Journal International, 132, p. 302-338.

\section{CH-06, WESTERN BACK THRUST FAULT OF CERRO DE PURILACTIS}

FAULT NUMBER/NUMERO DE LA FALLA: CH-06

FAULT NAME/NOMBRE DE LA FALLA: Western Back Thrust of Cerro de Purilactis

SYNOPSIS AND GEOLOGIC SETTING/SINOPSIS Y AMBIENTE GEOLOGICO: Quaternary activity described in 1990 (Jolley and others, 1990). General tectonic field studies. The fault trace is continuous and has placed the Upper Cretaceous Purilactis Formation over a Tertiary and Pleistocene sequence, west of the Cerros de Purilactis.

Actividad Cuaternaria descrita en 1990 (Jolley and others, 1990). Estudios tectónicos de campo generales. La traza de la falla es contínua y hace cabalgar la formación Purilactis del Cretácico superior sobre una secuencia terciaria y pleistocena, al oeste de los Cerros Purilactis.

COMPILER, AFFILIATION, \& DATE OF COMPILATION/COMPILADOR, AFILIACION Y FECHA DE

COMPILACION: Alain Lavenu, IRD, France, and RicardoThiele, Univ. de Chile; Chile, 08-1997.

TYPE OF STUDIES/TIPOS DE ESTUDIOS: Fieldwork/Trabajos de campo

FAULT GEOMETRY/GEOMETRIA DE LA FALLA:

LENGTH/LONGITUD: $15.6 \mathrm{~km}$ (15.6 km cumulative)

AVERAGE STRIKE/RUMBO PROMEDIO: $27.0^{\circ} \pm 18^{\circ}\left(\mathrm{N} 27.0^{\circ} \mathrm{E} \pm 18^{\circ}\right)$

AVERAGE DIP/INCLINACION PROMEDIO: $45^{\circ} \mathrm{E}$ in cross section $/ 45^{\circ} \mathrm{E}$ en perfil

SENSE OF MOVEMENT/SENTIDO DE MOVIMIENTO: Thrust/Inverso

Comments/Comentarios: This fault is more than $20 \mathrm{~km}$ long, trending NNE to NE

Falla de más de $20 \mathrm{~km}$ de largo, de dirección promedio NNE a NE

GEOMORPHIC EXPRESSION/EXPRESION GEOMORFOLOGICA: Unknown/desconocida

SLIP RATE/TASA DE MOVIMIENTO: Unknown/desconocida

TIME OF MOST RECENT OF MOVEMENT/EDAD DEL ULTIMO MOVIMIENTO: Quaternary/Cuaternario $(<1.6 \mathrm{Ma})$ 
REFERENCES/REFERENCIAS

Jolley, E.J., Turner, P., Williams, G.D., Hartley, A.J., Flint, S., 1990, Sedimentological response of an alluvial system to Neogene thrust tectonics, Atacama Desert, northern Chile: Journal of the Geological Society, London, 147, p. 769-784.

\section{CH-07, FRONTAL DOMEYKO THRUST FAULT OF LLANO DE LA PACIENCIA}

FAULT NUMBER/NUMERO DE LA FALLA: $\mathrm{CH}-07$

FAULT NAME/NOMBRE DE LA FALLA: Frontal Domeyko Thrust of Llano de la Paciencia

SYNOPSIS AND GEOLOGIC SETTING/SINOPSIS Y AMBIENTE GEOLOGICO: Quaternary activity described in 1990 (Jolley and others, 1990). General tectonic field studies. The fault trace defines the western margin of the Llano de la Paciencia, and it places the Oligocene Paciencia Group over Quaternary-Recent gravels.

Actividad cuaternaria descrita en 1990 (Jolley and others, 1990). Estudios tectónicos de campo generales. La traza de la falla limita al oeste el Llano de la Paciencia y hace cabalgar el Grupo Paciencia ,de edad oligocena, sobre gravas del Cuaternario.

COMPILER, AFFILIATION, \& DATE OF COMPILATION/COMPILADOR, AFILIACION Y FECHA DE COMPILACION: Alain Lavenu, IRD, France, and RicardoThiele, Univ. de Chile; Chile, 08-1997.

TYPE OF STUDIES/TIPOS DE ESTUDIOS: Fieldwork/Trabajos de campo

FAULT GEOMETRY/GEOMETRIA DE LA FALLA:

LENGTH/LONGITUD: $19.6 \mathrm{~km}$ (20.2 km cumulative)

AVERAGE STRIKE/RUMBO PROMEDIO: $39.2^{\circ} \pm 19^{\circ}\left(\mathrm{N} 39.2^{\circ} \mathrm{E} \pm 19^{\circ}\right)$

AVERAGE DIP/INCLINACION PROMEDIO: $45^{\circ} \mathrm{W}$ in cross section $/ 45^{\circ} \mathrm{W}$ en perfil

SENSE OF MOVEMENT/SENTIDO DE MOVIMIENTO: Reverse/Inverso

Comments/Comentarios: This fault is more than $35 \mathrm{~km}$ long, trending NE

Falla de más de $35 \mathrm{~km}$ de largo, de dirección promedio NE

GEOMORPHIC EXPRESSION/EXPRESION GEOMORFOLOGICA: Unknown/desconocida

SLIP RATE/TASA DE MOVIMIENTO: Unknown/desconocida

TIME OF MOST RECENT OF MOVEMENT/EDAD DEL ULTIMO MOVIMIENTO: Quaternary/Cuaternario $(<1.6 \mathrm{Ma})$

REFERENCES/REFERENCIAS

Jolley, E.J., Turner, P., Williams, G.D., Hartley, A.J., Flint, S., 1990, Sedimentological response of an alluvial system to Neogene thrust tectonics, Atacama Desert, northern Chile: Journal of the Geological Society, London, 147, p. 769-784.

\section{CH-08, IGNIMBRITE BACK THRUST FAULT OF LLANO DE LA PACIENCIA}

\section{FAULT NUMBER/NUMERO DE LA FALLA: CH-08}

FAULT NAME/NOMBRE DE LA FALLA: Ignimbrite Back Thrust of Llano de la Paciencia

SYNOPSIS AND GEOLOGIC SETTING/SINOPSIS Y AMBIENTE GEOLOGICO: Quaternary activity described in 1990 (Jolley and others, 1990). General tectonic field studies. The morphological continuity of the fan alluvial lobe surface is disrupted and uplifted by the synsedimentary effects of the Ignimbrite Back Thrust (IBT) (Jolley and others, 1990).

Actividad cuaternaria descrita en 1990 (Jolley and others, 1990). Estudios tectónicos de campo generales. La continuidad morfológica de los lóbulos de los abanicos aluviales se encuentra cortada y levantada por los efectos sinsedimentarios del IBT (Jolley and others, 1990).

COMPILER, AFFILIATION, \& DATE OF COMPILATION/COMPILADOR, AFILIACION Y FECHA DE COMPILACION: Alain Lavenu, IRD, France, and RicardoThiele, Univ. de Chile; Chile, 08-1997.

TYPE OF STUDIES/TIPOS DE ESTUDIOS: Fieldwork and interpretation of aerial photographs.

Trabajos de campo y fotointerpretación. 
FAULT GEOMETRY/GEOMETRIA DE LA FALLA:

LENGTH/LONGITUD: $6.9 \mathrm{~km}$ (6.9 km cumulative)

AVERAGE STRIKE/RUMBO PROMEDIO: $35.2^{\circ} \pm 18^{\circ}\left(\mathrm{N} 35.2^{\circ} \mathrm{E} \pm 18^{\circ}\right)$

AVERAGE DIP/INCLINACION PROMEDIO: E

SENSE OF MOVEMENT/SENTIDO DE MOVIMIENTO: Reverse/Inverso

Comments/Comentarios: This active structure is more than $4 \mathrm{~km}$ long, trending NNE.

Este estructura activo tiene más de $4 \mathrm{~km}$ de largo, de dirección promedio NNE.

GEOMORPHIC EXPRESSION/EXPRESION GEOMORFOLOGICA: Presence of gullies and ponds along

the structural strike of the IBT due to its continued uplift.

Presencia de depresiones y charcos a lo largo de la dirección estructural del IBT debidos al

levantamiento contínuo.

SLIP RATE/TASA DE MOVIMIENTO: Unknown/desconocida

TIME OF MOST RECENT OF MOVEMENT/EDAD DEL ULTIMO MOVIMIENTO: Quaternary/Cuaternario $(<1.6 \mathrm{Ma})$

REFERENCES/REFERENCIAS

Jolley, E.J., Turner, P., Williams, G.D., Hartley, A.J., Flint, S., 1990, Sedimentological response of an alluvial system to Neogene thrust tectonics, Atacama Desert, northern Chile: Journal of the Geological Society, London, 147, p. 769-784.

\section{CH-09, EASTERN FRONTAL THRUST FAULT OF LLANO DE LA PACIENCIA}

FAULT NUMBER/NUMERO DE LA FALLA: CH-09

FAULT NAME/NOMBRE DE LA FALLA: Eastern Frontal Thrust Fault of Llano de la Paciencia

SYNOPSIS AND GEOLOGIC SETTING/SINOPSIS Y AMBIENTE GEOLOGICO: Quaternary activity

described in 1990 (Jolley and others, 1990). General tectonic field studies. The morphological

continuity of the fan alluvial lobe surface is uplifted by the synsedimentary effects of the Eastern Frontal Thrust (EFT) (Jolley and others, 1990).

Actividad cuaternaria descrita en 1990 (Jolley and others, 1990). Estudios tectónicos de campo generales. La continuidad morfológica de los lóbulos de los abanicos aluviales se encuentra levantada por los efectos sinsedimentarios del EFT (Jolley and others, 1990).

COMPILER, AFFILIATION, \& DATE OF COMPILATION/COMPILADOR, AFILIACION Y FECHA DE

COMPILACION: Alain Lavenu, IRD, France, and RicardoThiele, Univ. de Chile; Chile, 08-1997.

TYPE OF STUDIES/TIPOS DE ESTUDIOS: Fieldwork and interpretation of aerial photographs.

Trabajos de campo y fotointerpretación.

FAULT GEOMETRY/GEOMETRIA DE LA FALLA:

LENGTH/LONGITUD: $14.9 \mathrm{~km}$ (15.3 km cumulative)

AVERAGE STRIKE/RUMBO PROMEDIO: $4.9^{\circ} \pm 23^{\circ}\left(\mathrm{N} 4.9^{\circ} \mathrm{E} \pm 23^{\circ}\right)$

AVERAGE DIP/INCLINACION PROMEDIO: Unknown angle to W/Desconocida al W

SENSE OF MOVEMENT/SENTIDO DE MOVIMIENTO: Reverse/Inverso

Comments/Comentarios: This active structure is more than $15 \mathrm{~km}$ long, trending N-S.

El estructura activo de la falla tiene más de $15 \mathrm{~km}$ de largo, de dirección promedio N-S.

GEOMORPHIC EXPRESSION/EXPRESION GEOMORFOLOGICA:.

SLIP RATE/TASA DE MOVIMIENTO: Unknown/desconocida

TIME OF MOST RECENT OF MOVEMENT/EDAD DEL ULTIMO MOVIMIENTO: Quaternary/Cuaternario $(<1.6 \mathrm{Ma})$

REFERENCES/REFERENCIAS

Jolley, E.J., Turner, P., Williams, G.D., Hartley, A.J., Flint, S., 1990, Sedimentological response of an alluvial system to Neogene thrust tectonics, Atacama Desert, northern Chile: Journal of the Geological Society, London, 147, p. 769-784. 


\section{CH-10, FRONTAL THRUST FAULT OF CORDILLERA DE LA SAL}

FAULT NUMBER/NUMERO DE LA FALLA: CH-10

FAULT NAME/NOMBRE DE LA FALLA: Frontal Thrust of Cordillera de la Sal

SYNOPSIS AND GEOLOGIC SETTING/SINOPSIS Y AMBIENTE GEOLOGICO: Quaternary activity

described in 1990 (Jolley and others, 1990). General tectonic field studies. The morphological continuity of the alluvial plain of the Salar de Atacama has been progressively deformed by the synsedimentary effects of the Cordillera de la Sal Frontal Thrust (CDSFT) (Jolley and others, 1990). Actividad cuaternaria descrita en 1990 (Jolley and others, 1990). Estudios tectónicos de campo generales. La continuidad morfológica de la planicie aluvial del Salar de Atacama ha sido deformada por los efectos sinsedimentarios del CDSFT (Jolley and others, 1990).

COMPILER, AFFILIATION, \& DATE OF COMPILATION/COMPILADOR, AFILIACION Y FECHA DE

COMPILACION: Alain Lavenu, IRD, France, and RicardoThiele, Univ. de Chile; Chile, 08-1997.

TYPE OF STUDIES/TIPOS DE ESTUDIOS: Fieldwork and interpretation of aerial photographs.

Trabajos de campo y fotointerpretación.

FAULT GEOMETRY/GEOMETRIA DE LA FALLA:

LENGTH/LONGITUD: $34.7 \mathrm{~km}$ (36.5 km cumulative)

AVERAGE STRIKE/RUMBO PROMEDIO: $42.3^{\circ} \pm 19^{\circ}\left(\mathrm{N} 42.3^{\circ} \mathrm{E} \pm 19^{\circ}\right)$

AVERAGE DIP/INCLINACION PROMEDIO: Unknown angle to W/Desconocida al W

SENSE OF MOVEMENT/SENTIDO DE MOVIMIENTO: Reverse/Inverso

Comments/Comentarios: This fault is more than $30 \mathrm{~km}$ long, trending NE.

La falla tiene más de $30 \mathrm{~km}$ de largo, de dirección promedio NE.

GEOMORPHIC EXPRESSION/EXPRESION GEOMORFOLOGICA: Unknown/desconocida

SLIP RATE/TASA DE MOVIMIENTO: Unknown/desconocida

TIME OF MOST RECENT OF MOVEMENT/EDAD DEL ULTIMO MOVIMIENTO: Quaternary/Cuaternario $(<1.6 \mathrm{Ma})$

REFERENCES/REFERENCIAS

Jolley, E.J., Turner, P., Williams, G.D., Hartley, A.J., Flint, S., 1990, Sedimentological response of an alluvial system to Neogene thrust tectonics, Atacama Desert, northern Chile: Journal of the Geological Society, London, 147, p. 769-784.

\section{CH-11, TUCUCARO FAULT}

FAULT NUMBER/NUMERO DE LA FALLA: $\mathrm{CH}-11$

FAULT NAME/NOMBRE DE LA FALLA: Tucúcaro

SYNOPSIS AND GEOLOGIC SETTING/SINOPSIS Y AMBIENTE GEOLOGICO: Quaternary activity described in 1984 (Niemeyer, 1984). General tectonic field studies. This fault defines the eastern margin of the Cordon de Lila and the western margins of the Pampas de los Perdidos and El Jilguero, south of the Salar de Atacama. The last motion of the fault affects the Ignimbrite Tucúcaro (3.2 $\pm 0.3 \mathrm{Ma}$, Ramirez y Gardeweg, 1982) and Quaternary aluvial deposits, but not younger aluvial fans. Actividad Cuaternaria descrita en 1990 (Jolley and others, 1990). Estudios tectónicos de campo generales. Esta falla limita al este el Cordon de Lila y al oeste las Pampas de los Perdidos y El Jilguero, al sur del Salar de Atacama. El último movimiento de la falla afecta a la Ignimbrita Tucúcaro (3.2 $\pm 0.3 \mathrm{Ma}$, Ramirez y Gardeweg, 1982) y depósitos aluviales cuaternarios pero no a abanicos aluviales más recientes.

COMPILER, AFFILIATION, \& DATE OF COMPILATION/COMPILADOR, AFILIACION Y FECHA DE COMPILACION: Alain Lavenu, IRD, France, and RicardoThiele, Univ. de Chile; Chile, 08-1997.

TYPE OF STUDIES/TIPOS DE ESTUDIOS: Fieldwork and interpretation of aerial photographs.

Trabajos de campo y fotointerpretación. 
FAULT GEOMETRY/GEOMETRIA DE LA FALLA:

LENGTH/LONGITUD: $30.2 \mathrm{~km}$ (28.7 km cumulative)

AVERAGE STRIKE/RUMBO PROMEDIO: $8.2^{\circ} \pm 20^{\circ}\left(\mathrm{N} 8.2^{\circ} \mathrm{E} \pm 20^{\circ}\right)$

AVERAGE DIP/INCLINACION PROMEDIO: $50^{\circ} \mathrm{W}$

SENSE OF MOVEMENT/SENTIDO DE MOVIMIENTO: Reverse/Inverso

Comments/Comentarios: This fault is more than $25 \mathrm{~km}$ long, trending N-S to NNE.

El falla tiene más de $25 \mathrm{~km}$ de largo, de dirección promedio N-S a NNE.

GEOMORPHIC EXPRESSION/EXPRESION GEOMORFOLOGICA: The scarp faces toward the east and the throw is of approx. $3 \mathrm{~m}$.

El escarpe de falla es hacia el este y el rechazo es de unos 3 metros.

SLIP RATE/TASA DE MOVIMIENTO: Unknown/desconocida

TIME OF MOST RECENT OF MOVEMENT/EDAD DEL ULTIMO MOVIMIENTO: Quaternary/Cuaternario $(<1.6 \mathrm{Ma})$

REFERENCES/REFERENCIAS

Niemeyer, H., 1984, La mega falla Tucúcaro en el extremo sur del Salar de Atacama: una antigua zona de cizalle reactivada en el Cenozoico: Comunicaciones, 34, p. 37-45, Univ. de Chile, Santiago.

\section{CH-12, FAULTS SOUTH OF CALDERA}

\section{FAULT NUMBER/NUMERO DE LA FALLA: $\mathrm{CH}-12$}

FAULT NAME/NOMBRE DE LA FALLA: South of Caldera (informal name)/Sur de Caldera (sin nombre)

SYNOPSIS AND GEOLOGIC SETTING/SINOPSIS Y AMBIENTE GEOLOGICO: Quaternary activity described in 1999 (Marquardt, 1999). General neotectonic field studies. The main fault is $7 \mathrm{~km}$ long and affects marine deposits (Quaternary terrace assigned to the $220 \mathrm{ka}$ marine high stand, i.e. the isotopic stage 7 and Quaternary terrace assigned to the $125 \mathrm{ka}$ marine high stand, i.e., the isotopic stage 5). Southward, two other normal faults are parallel to the main fault. A NNE-trending secondary antithetic splay, $1.5 \mathrm{~km}$ long, linked with the main fault, suggests a small graben related to a NW-SE extension. Actividad Cuaternaria descrita en 1999 (Marquardt, 1999). Estudios neotectónicos de campo generales. La falla principal tiene una longitud de $7 \mathrm{~km}$ y afecta a depósitos marinos (terraza cuaternaria asignada al alto nivel marino de $225 \mathrm{ka}$, i.e., el estado isotópico 7 y terraza cuaternaria asignada al alto nivel marino de $125 \mathrm{ka}$, i.e. el estado isotópico 5). Hacia el sur, dos otras fallas normales son parallelas a esta falla principal. Una falla segundaria antitética de dirección NNE, de $1.5 \mathrm{~km}$ de largo, sugiere un pequeño graben ligado a una extensión NW-SE.

COMPILER, AFFILIATION, \& DATE OF COMPILATION/COMPILADOR, AFILIACION Y FECHA DE COMPILACION: Alain Lavenu, IRD, France, and RicardoThiele, Univ. de Chile; Chile, 08-1997.

TYPE OF STUDIES/TIPOS DE ESTUDIOS: Fieldwork and interpretation of aerial photographs.

Trabajos de campo y fotointerpretación.

FAULT GEOMETRY/GEOMETRIA DE LA FALLA:

LENGTH/LONGITUD: $6.4 \mathrm{~km}$ (11.6 km cumulative)

AVERAGE STRIKE/RUMBO PROMEDIO: $52.0^{\circ} \pm 14^{\circ}\left(\mathrm{N} 52.0^{\circ} \mathrm{E} \pm 14^{\circ}\right)$

AVERAGE DIP/INCLINACION PROMEDIO: $65^{\circ} \mathrm{NW}$

SENSE OF MOVEMENT/SENTIDO DE MOVIMIENTO: Normal

Comments/Comentarios: The principal fault is more than $7 \mathrm{~km}$ long, trending NNE.

La falla principal tiene más de $7 \mathrm{~km}$ de largo, de dirección promedio NNE.

GEOMORPHIC EXPRESSION/EXPRESION GEOMORFOLOGICA: The scarp faces toward the northwest and the throw is approximately $3 \mathrm{~m}$.

El escarpe de falla es hacia el nor-oeste y el rechazo es de unos 3 metros.

RECURRENCE INTERVAL/INTERVALO DE RECURRENCIA: Unknown/desconocido

SLIP RATE/TASA DE MOVIMIENTO: $0.30 \mathrm{~mm} / \mathrm{yr}$ (0.30 mm/año)

TIME OF MOST RECENT OF MOVEMENT/EDAD DEL ULTIMO MOVIMIENTO: Quaternary (<1.6 Ma)

Comments/Comentarios: Classified as Quatenary, but most recent movement is $125 \mathrm{ka}$.

Cuaternairo, pero edad del ultimo movimiento es $125 \mathrm{ka}$. 
REFERENCES/REFERENCIAS

Marquardt, C., 1999, Neotectónica de la franja costera y aportes a la geología regional entre Caldera y Caleta Pajonal

$\left(27^{\circ} 00^{\prime}-27^{\circ} 45^{\prime}\right)$, III Región de Atacama: Unpublished thesis, University of Chile, Santiago, 297 p.

\section{CH-13, FAULTS EAST OF BAHIA INGLESA}

FAULT NUMBER/NUMERO DE LA FALLA: $\mathrm{CH}-13$

FAULT NAME/NOMBRE DE LA FALLA: East of Bahía Inglesa (informal name)/ Est de Bahía Inglesa (sin nombre)

SYNOPSIS AND GEOLOGIC SETTING/SINOPSIS Y AMBIENTE GEOLOGICO: Quaternary activity described in 1999 (Marquardt, 1999). General neotectonic field studies. The fault is $2.5 \mathrm{~km}$ long and affects marine deposits younger than $125 \mathrm{ka}$ (Quaternary terraces assigned to the isotopic stage 5). This normal fault is compatible with a WNW-ESE extension.

Actividad Cuaternaria descrita en 1999 (Marquardt, 1999). Estudios neotectónicos de campo generales. La falla tiene una longitud de $2.5 \mathrm{~km}$ y afecta a depósitos marinos más recientes que $125 \mathrm{ka}$ (terraza Cuaternaria asignada al estado isotópico 5). Esta falla normal es compatible con una extensión WNW-ESE.

COMPILER, AFFILIATION, \& DATE OF COMPILATION/COMPILADOR, AFILIACION Y FECHA DE COMPILACION: Alain Lavenu, IRD, France, and RicardoThiele, Univ. de Chile; Chile, 08-1997.

TYPE OF STUDIES/TIPOS DE ESTUDIOS: Fieldwork and interpretation of aerial photographs. Trabajos de campo y fotointerpretación.

FAULT GEOMETRY/GEOMETRIA DE LA FALLA:

LENGTH/LONGITUD: $2.7 \mathrm{~km}$ (2.5 km cumulative)

AVERAGE STRIKE/RUMBO PROMEDIO: $21.0^{\circ} \pm 21^{\circ}\left(\mathrm{N} 21.0^{\circ} \mathrm{E} \pm 21^{\circ}\right)$

AVERAGE DIP/INCLINACION PROMEDIO: $80^{\circ} \mathrm{W}$ in the northern part and $80^{\circ} \mathrm{E}$ in the southern part $/ 80^{\circ} \mathrm{W}$ en el parte norte y $80^{\circ} \mathrm{E}$ en el parte sur.

SENSE OF MOVEMENT/SENTIDO DE MOVIMIENTO: Normal

Comments/Comentarios: The fault is $2.5 \mathrm{~km}$ long, trending NNE.

La falla tiene $2.5 \mathrm{~km}$ de largo, de dirección promedio NNE.

GEOMORPHIC EXPRESSION/EXPRESION GEOMORFOLOGICA: The scarp faces westward in its northern half and eastward in the southern half, corresponding to a scissors fault. Maximum vertical offset is up to $4 \mathrm{~m}$.

El escarpe de falla es hacia el oeste en el mo norte y hacia el este en la parte sur, lo que corresponde a una falla en tijera. El rechazo máximo es de $4 \mathrm{~m}$.

RECURRENCE INTERVAL/INTERVALO DE RECURRENCIA: Unknown/desconocido

SLIP RATE/TASA DE MOVIMIENTO: $0.27 \mathrm{~m} / \mathrm{ka}(0.27 \mathrm{~mm} / \mathrm{yr})$

TIME OF MOST RECENT OF MOVEMENT/EDAD DEL ULTIMO MOVIMIENTO: Quaternary (<1.6 Ma)

Comments/Comentarios: Classified as Quatenary, but most recent movement is $125 \mathrm{ka}$.

Cuaternairo, pero edad del ultimo movimiento es $125 \mathrm{ka}$.

REFERENCES/REFERENCIAS

Marquardt, C., 1999, Neotectónica de la franja costera y aportes a la geología regional entre Caldera y Caleta Pajonal $\left(27^{\circ} 00^{\prime}-27^{\circ} 45^{\prime}\right)$, III Región de Atacama: Unpublished thesis, University of Chile, Santiago, 297 p.

\section{CH-14, FAULTS OF ALTO DEL FRAILE}

FAULT NUMBER/NUMERO DE LA FALLA: $\mathrm{CH}-14$

FAULT NAME/NOMBRE DE LA FALLA: Alto del Fraile (informal name)/ Alto del Fraile (sin nombre) SYNOPSIS AND GEOLOGIC SETTING/SINOPSIS Y AMBIENTE GEOLOGICO: Quaternary activity described in 1999 (Marquardt, 1999). General neotectonic field studies. Morphological scarps are linked to two groups of normal faults, trending NW-SE and NE-SW. The scarps affect Quaternary marine deposits, but the fault planes are not observed.

Actividad Cuaternaria descrita en 1999 (Marquardt, 1999). Estudios neotectónicos de campo generales. Escarpes morfológicos se relacionan a dos grupos de fallas normales de dirección NW-SE y NE-SW. Los escarpes afectan depositos marinos cuaternarios pero no se observan los planos de falla. 
COMPILER, AFFILIATION, \& DATE OF COMPILATION/COMPILADOR, AFILIACION Y FECHA DE COMPILACION: Alain Lavenu, IRD, France, and RicardoThiele, Univ. de Chile; Chile, 08-1997.

TYPE OF STUDIES/TIPOS DE ESTUDIOS: Fieldwork and interpretation of aerial photographs.

Trabajos de campo y fotointerpretación.

FAULT GEOMETRY/GEOMETRIA DE LA FALLA:

LENGTH/LONGITUD: $9.5 \mathrm{~km}$ (24.0 km cumulative)

AVERAGE STRIKE/RUMBO PROMEDIO: $-4.2^{\circ} \pm 55^{\circ}\left(\mathrm{N} 4.2^{\circ} \mathrm{W} \pm 55^{\circ}\right)$

AVERAGE DIP/INCLINACION PROMEDIO: Unknown/Desconocida

SENSE OF MOVEMENT/SENTIDO DE MOVIMIENTO: Normal

GEOMORPHIC EXPRESSION/EXPRESION GEOMORFOLOGICA: Morphological scarps only are observed./Solamente se observan escarpes morfológicos.

RECURRENCE INTERVAL/INTERVALO DE RECURRENCIA: Unknown/desconocido

SLIP RATE/TASA DE MOVIMIENTO: Unknown/desconocida

TIME OF MOST RECENT OF MOVEMENT/EDAD DEL ULTIMO MOVIMIENTO: Quaternary/Cuaternario $(<1.6 \mathrm{Ma})$

REFERENCES/REFERENCIAS

Marquardt, C., 1999, Neotectónica de la franja costera y aportes a la geología regional entre Caldera y Caleta Pajonal $\left(27^{\circ} 00^{\prime}-27^{\circ} 45^{\prime}\right)$, III Región de Atacama: Unpublished thesis, University of Chile, Santiago, 297 p.

\section{CH-15, FAULT OF SAN JOSE DE MAIPO}

FAULT NUMBER/NUMERO DE LA FALLA: $\mathrm{CH}-15$

FAULT NAME/NOMBRE DE LA FALLA: San Jose de Maipo (informal name)/ San Jose de Maipo (sin nombre)

SYNOPSIS AND GEOLOGIC SETTING/SINOPSIS Y AMBIENTE GEOLOGICO: Quaternary activity. Not described in detail. General neotectonic field studies. Reverse fault affects a high terrace (t5) of the Maipo River, $30 \mathrm{~km}$ south-east of Santiago. The age of these fluvial deposits are unknown and could be of middle Pleistocene. The fault does not cut the topographic surface of the terrace. Several small reverse faults are parallel to the main fault plane. The reverse faults are compatible with a N-S-trending compressional deformation.

Actividad Cuaternaria. No descrita en detalle. Estudios neotectónicos de campo generales. Una falla inversa afecta una alta terraza (t5) del Rió Maipo, $30 \mathrm{~km}$ al sur-este de Santiago. La edad de estos depositos fluviatiles no se conoce y podría ser del Pleistoceno medio. La falla no cortan la superficie topográfica de la terraza. Otras pequeñas fallas inversas son paralelas a la traza de la falla principal. Las fallas inversas son compatibles con una compresión de dirección N-S.

COMPILER, AFFILIATION, \& DATE OF COMPILATION/COMPILADOR, AFILIACION Y FECHA DE COMPILACION: Alain Lavenu, IRD, France, and RicardoThiele, Univ. de Chile; Chile, 08-1997.

TYPE OF STUDIES/TIPOS DE ESTUDIOS: Fieldwork, interpretation of aerial photographs, numerical analysis of the faults.

Trabajos de campo, fotointerpretación, análisis numérico de las fallas.

FAULT GEOMETRY/GEOMETRIA DE LA FALLA:

LENGTH/LONGITUD: $1.7 \mathrm{~km}$ (1.7 km cumulative)

AVERAGE STRIKE/RUMBO PROMEDIO: $83.0^{\circ} \pm 0^{\circ}\left(\mathrm{N} 83.0^{\circ} \mathrm{E} \pm 0^{\circ}\right)$

AVERAGE DIP/INCLINACION PROMEDIO: $65^{\circ} \mathrm{N}$

SENSE OF MOVEMENT/SENTIDO DE MOVIMIENTO: Reverse/Inverso

GEOMORPHIC EXPRESSION/EXPRESION GEOMORFOLOGICA: Unknown/desconocida

SLIP RATE/TASA DE MOVIMIENTO: Unknown/desconocida

TIME OF MOST RECENT OF MOVEMENT/EDAD DEL ULTIMO MOVIMIENTO: Quaternary/Cuaternario $(<1.6 \mathrm{Ma})$

REFERENCES/REFERENCIAS

Lavenu, A., and Cembrano, J., 1999, Compressional- and transpressional-stress pattern for Pliocene and

Quaternary brittle deformation in fore arc and intra-arc zones (Andes of Central and Southern Chile): Journal of Structural Geology, v. 21, p. 1669-1691. 


\section{CH-16, FAULT OF ESPERANZA}

FAULT NUMBER/NUMERO DE LA FALLA: CH-16

FAULT NAME/NOMBRE DE LA FALLA: Esperanza (informal name)/ Esperanza (sin nombre)

SYNOPSIS AND GEOLOGIC SETTING/SINOPSIS Y AMBIENTE GEOLOGICO: Quaternary activity described in 1999 (Lavenu and Cembrano, 1999). General neotectonic field studies. A reverse fault affects a high terrace of the Renaico River, $40 \mathrm{~km}$ south of Los Angeles. Varela and Moreno (1982) considered the age of the fluvial deposits in this region of the Central Depression as Middle Pleistocene (0.7 Ma) (Unidad del Cono de Quilleco after Thiele and others, 1998). The fault does not cut the topographic surface of the terrace. Several small reverse faults are parallel or antithetic to the main fault plane. These reverse faults are compatible with a N-S compressional deformation.

Actividad Cuaternaria. No descrita. Estudios neotectónicos de campo generales. Una falla inversa afecta una alta terraza del Rió Renaico, $40 \mathrm{~km}$ al sur de Los Angeles. Varela y Moreno (1982) estiman la edad de estos depositos fluviatiles, en esta zona de la Depresión Central, como del Pleistoceno medio (0.7 Ma) (Unidad del Cono de Quilleco según Thiele and others, 1998). La falla no corta la superficie topográfica de la terraza. Otras pequeñas fallas inversas son paralelas o antitéticas a la traza de la falla principal. Estas fallas inversas son compatibles con una compresión de dirección N-S.

COMPILER, AFFILIATION, \& DATE OF COMPILATION/COMPILADOR, AFILIACION Y FECHA DE COMPILACION: Alain Lavenu, IRD, France, and RicardoThiele, Univ. de Chile; Chile, 08-1997.

TYPE OF STUDIES/TIPOS DE ESTUDIOS: Fieldwork, interpretation of aerial photographs, numerical analysis of the faults.

Trabajos de campo, fotointerpretación, análisis numérico de las fallas.

FAULT GEOMETRY/GEOMETRIA DE LA FALLA:

LENGTH/LONGITUD: $2.7 \mathrm{~km}$ (2.7 km cumulative)

AVERAGE STRIKE/RUMBO PROMEDIO: $64.9^{\circ} \pm 8^{\circ}\left(\mathrm{N} 64.9^{\circ} \mathrm{E} \pm 9^{\circ}\right)$

AVERAGE DIP/INCLINACION PROMEDIO: $65^{\circ} \mathrm{S}$

SENSE OF MOVEMENT/SENTIDO DE MOVIMIENTO: Reverse/Inverso

GEOMORPHIC EXPRESSION/EXPRESION GEOMORFOLOGICA: Unknown/desconocida

SLIP RATE/TASA DE MOVIMIENTO: Unknown/desconocida

TIME OF MOST RECENT OF MOVEMENT/EDAD DEL ULTIMO MOVIMIENTO: Quaternary/Cuaternario $(<1.6 \mathrm{Ma})$

REFERENCES/REFERENCIAS

Lavenu, A., and Cembrano, J., 1999, Compressional- and transpressional-stress pattern for Pliocene and

Quaternary brittle deformation in fore arc and intra-arc zones (Andes of Central and Southern Chile): Journal of

Structural Geology, v. 21, p. 1669-1691.

Lavenu, A., Cembrano, J, 1999, Estados de esfuerzo compresivo plioceno y compresivo-transpresivo pleistoceno, Andes del sur, Chile (38-42 30 'S): Revista Geológica de Chile, 26, 1, p. 67-87.

\section{CH-17, FAULT OF VICTORIA}

FAULT NUMBER/NUMERO DE LA FALLA: $\mathrm{CH}-17$

FAULT NAME/NOMBRE DE LA FALLA: Victoria (informal name)/ Victoria (sin nombre)

SYNOPSIS AND GEOLOGIC SETTING/SINOPSIS Y AMBIENTE GEOLOGICO: Quaternary activity described in 1999 (Lavenu and Cembrano, 1999). General neotectonic field studies. A reverse fault affects a high terrace of the Traiguen River, $2 \mathrm{~km}$ north of Victoria. Varela and Moreno (1982)

considered the age of the fluvial deposits in this region of the Central Depression as Middle Pleistocene (0.7 Ma) (Unidad del Cono aluvial de Malleco, equivalent to the "Rodados Multicolores" after Hauser, 1986). The fault does not cut the topographic surface of the terrace. Other small reverse faults are parallel to the main fault plane. These reverse faults are compatible with a N-S compressional deformation.

Actividad Cuaternaria. No descrita. Estudios neotectónicos de campo generales. Una falla inversa afecta una alta terraza del Rió Traiguen, $2 \mathrm{~km}$ al norte de Victoria. Varela y Moreno (1982) estiman la edad de estos depositos fluviatiles, en esta zona de la Depresión Central, como del Pleistoceno medio $(0.7 \mathrm{Ma})$ (Unidad del Cono aluvial de Malleco, equivalente de los "Rodados Multicolores" de Hauser, 1986).). La falla no cortan la superficie topográfica de la terraza. Otras pequeñas fallas inversas son 
paralelas a la traza de la falla principal. Estas fallas inversas son compatibles con una compresión de dirección N-S.

COMPILER, AFFILIATION, \& DATE OF COMPILATION/COMPILADOR, AFILIACION Y FECHA DE COMPILACION: Alain Lavenu, IRD, France, and RicardoThiele, Univ. de Chile; Chile, 08-1997.

TYPE OF STUDIES/TIPOS DE ESTUDIOS: Fieldwork, interpretation of aerial photographs, numerical analysis of the faults.

Trabajos de campo, fotointerpretación, análisis numérico de las fallas.

FAULT GEOMETRY/GEOMETRIA DE LA FALLA:

LENGTH/LONGITUD: $11.4 \mathrm{~km}$ (11.4 km cumulative)

AVERAGE STRIKE/RUMBO PROMEDIO: $-27.5^{\circ} \pm 6^{\circ}\left(\mathrm{N} 27.5^{\circ} \mathrm{W} \pm 6^{\circ}\right)$

AVERAGE DIP/INCLINACION PROMEDIO: $50^{\circ} \mathrm{NE}$

SENSE OF MOVEMENT/SENTIDO DE MOVIMIENTO: Reverse/Inverso

GEOMORPHIC EXPRESSION/EXPRESION GEOMORFOLOGICA: Unknown/desconocida

SLIP RATE/TASA DE MOVIMIENTO: Unknown/desconocida

TIME OF MOST RECENT OF MOVEMENT/EDAD DEL ULTIMO MOVIMIENTO: Quaternary/Cuaternario $(<1.6 \mathrm{Ma})$

REFERENCES/REFERENCIAS

Lavenu, A., and Cembrano, J., 1999, Compressional- and transpressional-stress pattern for Pliocene and

Quaternary brittle deformation in fore arc and intra-arc zones (Andes of Central and Southern Chile): Journal of Structural Geology, v. 21, p. 1669-1691.

Lavenu, A., Cembrano, J, 1999, Estados de esfuerzo compresivo plioceno y compresivo-transpresivo pleistoceno, Andes del sur, Chile (38-42³0'S): Revista Geológica de Chile, 26, 1, p. 67-87.

\section{CH-18, LIQUIÑE-OFQUI FAULT ZONE}

\section{FAULT NUMBER/NUMERO DE LA FALLA: $\mathrm{CH}-18$}

FAULT NAME/NOMBRE DE LA FALLA: Liquiñe-Ofqui

SYNOPSIS AND GEOLOGIC SETTING/SINOPSIS Y AMBIENTE GEOLOGICO: The regional-scale

geometry of the N-S Liquiñe-Ofqui fault zone (LOFZ), more than $950 \mathrm{~km}$ long, is characterized by two major NNE-trending sections (the eastern section is the only one obvious, the western being along the Moraleda Channel), joined by a series of an échelon NE-trending lineaments at a right step (Hervé and Thiele, 1987; Hervé, 1994). The resulting arrangement has been interpreted as a strike-slip duplex (Cembrano and others, 1996). Along the LOFZ, high strain zones show penetrative foliation in the North Patagonian batholith and metamorphic wall rocks (late Miocene and early Pliocene right lateral to reverse right lateral motions). Superposed on the ductile fabric, an anastomosing network of fractures and faults occurs along conspicuous N-trending lineaments (Pliocene to Quaternary transpressional right lateral motion, Cembrano, 1998; Lavenu and Cembrano, 1999). Quaternary volcanoes are aligned along the faults as well as in northeast- and northwest-trending groups oblique to the fault zone. In the extreme northern part of the LOFZ, near the Lonquimay Volcano $\left(38^{\circ} 22.5^{\prime} \mathrm{S} / 71^{\circ} 35.5^{\prime} \mathrm{W}\right)$ earthquakes were recorded following the December 25, 1988 eruption. Their focal mechanism is compatible with a $\mathrm{N}-\mathrm{S}$ trending dextral strike-slip fault, the motion resulting of a NNE-trending maximum compressional stress. In the extreme southern part of the LOFZ, near the Hudson Volcano, $25 \mathrm{~km}$ east of the principal fault trace, a shallow crustal earthquake was recorded on November 28, 1965. The focal mechanism solution is a dextral-reverse fault trending NNE-SSW, compatible to Quaternary paleostress. La geometría a escala regional de la Zona de Falla Liquiñe-Ofqui (ZFLO) de dirección N-S, y de más de 950 kmde largo, se caracteriza por dos seccionos mayores de dirección NNE (el secciono oriental es el más visible, el occidental siendo en el Canal de Moraleda), que se juntan con unas serie de lineamientos en échelon dextrales de dirección NE (Hervé and Thiele, 1987; Hervé, 1994). El arreglo resultante fue interpretado como un duplex de rumbo dextral (Cembrano and others, 1996). A lo largo de la ZFLO, zonas de alta deformación muestran una foliación penetrativa en las rocas del Batolito NorPatagónico y las rocas encajonantes metamórficas (movimientos de rumbo dextral a inverso dextral del Mioceno superior y Plioceno inferior). Superpuesta a la fábrica dúctil, una red anastomosada de fracturas y fallas ocurre a lo largo de conspicuos lineamientos de dirección N-S (movimiento de rumbo transpresional dextral Plioceno a Cuaternario, Cembrano, 1998; Lavenu y Cembrano, 1999). Volcanes 
cuaternarios se alignan a lo largo de fallas tanto en grupos de dirección NE como NW, oblicuos a la dirección de la zona de falla. En el extremo norte de la ZFLO, cerca del volcán Lonquimay $\left(38^{\circ} 22.5^{\prime} \mathrm{S} / 71^{\circ} 35.5^{\prime} \mathrm{W}\right)$ terremotos fueron registrados después de la erupción del 25 de diciembre de 1988: El mecanismo focal es compatible con una falla N-S de rumbo dextral, el movimiento resultando de un esfuerzo principal compresivo de dirección NNE-SSW. En el extremo sur de la ZFLO, cerca del volcán Hudson, $25 \mathrm{~km}$ al este de la traza principal de la falla, un terremoto cortical superficial fue registrado el 28 de noviembre de 1965. La solución del mecanismo focal indica una falla NNE-SSW dextral inversa, compatible con el paleoesfuerzo Cuaternario.

COMPILER, AFFILIATION, \& DATE OF COMPILATION/COMPILADOR, AFILIACION Y FECHA DE COMPILACION: Alain Lavenu, IRD, France, and RicardoThiele, Univ. de Chile; Chile, 08-1997.

TYPE OF STUDIES/TIPOS DE ESTUDIOS: See discussion under separate fault sections/Ver discusión abajo en las secciones de cada falla

FAULT GEOMETRY/GEOMETRIA DE LA FALLA:

LENGTH/LONGITUD: $696.6 \mathrm{~km}$ (412.5 km cumulative)

AVERAGE STRIKE/RUMBO PROMEDIO: $11.9^{\circ} \pm 13^{\circ}\left(\mathrm{N} 11.9^{\circ} \mathrm{E} \pm 13^{\circ}\right)$

AVERAGE DIP/INCLINACION PROMEDIO: Subvertical

SENSE OF MOVEMENT/SENTIDO DE MOVIMIENTO: Dextral

NUMBER OF SECTIONS/NOMBRE DE LA SECCIONES: 4 [CH-18a, CH-18b, CH-18c, CH-18d], Comments/Comentarios: For this compilation, four sections were defined for the Liquiñe-Ofqui fault Zone./Para esta compilación, cuatro secciones fueron definidas para la Zona de Falla Liquiñe-Ofqui.

\section{CH-18A, RELONCAVÍ FAULT, SECTION OF LIQUIÑE-OFQUI FAULT}

\section{SECTION NUMBER/NUMERO DE LA SECCION: CH-18a}

SECTION NAME/NOMBRE DE LA SECCION: Reloncaví fault (section of Liquiñe-Ofqui fault/seccion de falla Liquiñe-Ofqui)

SYNOPSIS AND GEOLOGIC SETTING/SINOPSIS Y AMBIENTE GEOLOGICO: Quaternary activity. General neotectonic field studies. Main Cordillera. This fault cuts sediment of Mesozoic to Neogene/Quaternary age. This fault is compatible with an NE-SW transpressional tectonic regime. Actividad Cuaternaria. Estudios neotectónicos de campo generales. Cordillera Principal. Corta sedimentos de edad mesozoica a neógena/cuaternaria. Esta falla es compatible con un régimen tectónico en transpresión NE-SW.

COMPILER, AFFILIATION, \& DATE OF COMPILATION/COMPILADOR, AFILIACION Y FECHA DE COMPILACION: Alain Lavenu, IRD, France, and RicardoThiele, Univ. de Chile; Chile, 08-1997.

TYPE OF STUDIES/TIPOS DE ESTUDIOS: Fieldwork and interpretation of aerial photographs.

Trabajos de campo y fotointerpretación.

SECTION GEOMETRY/GEOMETRIA DE LA SECCION:

LENGTH/LONGITUD: $90.2 \mathrm{~km}$ (60.0 km cumulative)

AVERAGE STRIKE/RUMBO PROMEDIO: $3.1^{\circ} \pm 5^{\circ}\left(\mathrm{N} 3.1^{\circ} \mathrm{E} \pm 5^{\circ}\right)$

AVERAGE DIP/INCLINACION PROMEDIO: Vertical

SENSE OF MOVEMENT/SENTIDO DE MOVIMIENTO: Dextral

Comments/Comentarios: This fault section is more than $30 \mathrm{~km}$ long, of average trend $\mathrm{N} 10^{\circ} \mathrm{E}$. Lateral and vertical displacements have yet to be quantified.

Falla de más de $30 \mathrm{~km}$, de dirección promedio $\mathrm{N} 10^{\circ} \mathrm{E}$. Los desplazamientos laterales y verticales no son todavia cuantificados.

GEOMORPHIC EXPRESSION/EXPRESION GEOMORFOLOGICA: This fault section is located in a very rainy region, impeding good observation of the fault's morphology. Uplift of the coast along this part of the fault zone was observed after the 1960 Valdivia earthquake.

Este seccion de la falla se encuentra en una zona muy lluviosa lo que impide una buena obsevación de la morfología de la falla. Se observó levantamiento de la costa en la zona de la falla posteriormente al sismo de Valdivia de 1960

RECURRENCE INTERVAL/INTERVALO DE RECURRENCIA: Unknown/desconocido

SLIP RATE/TASA DE MOVIMIENTO: Unknown/desconocida 
TIME OF MOST RECENT OF MOVEMENT/EDAD DEL ULTIMO MOVIMIENTO: Quaternary/Cuaternario $(<1.6 \mathrm{Ma})$

\section{CH-18B, HORNOPIRÉN FAULT, SECTION OF LIQUIÑE-OFQUI FAULT}

SECTION NUMBER/NUMERO DE LA SECCION: CH-18b

SECTION NAME/NOMBRE DE LA SECCION: Hornopirén fault (section of Liquiñe-Ofqui fault/seccion de falla Liquiñe-Ofqui)

SYNOPSIS AND GEOLOGIC SETTING/SINOPSIS Y AMBIENTE GEOLOGICO: Quaternary activity.

General neotectonic field studies. Main Cordillera. This fault cuts sediment of Mesozoic to

Neogene/Quaternary age. Compatible with an NE-SW transpressional tectonic regime.

Actividad Cuaternaria. Estudios neotectónicos de campo generales. Cordillera Principal. Corta sedimentos de edad mesozoica a neógena/cuaternaria. Esta falla es compatible con un régimen tectónico en transpresión NE-SW

COMPILER, AFFILIATION, \& DATE OF COMPILATION/COMPILADOR, AFILIACION Y FECHA DE

COMPILACION: Alain Lavenu, IRD, France, and RicardoThiele, Univ. de Chile; Chile, 08-1997.

TYPE OF STUDIES/TIPOS DE ESTUDIOS: Fieldwork and interpretation of aerial photographs.

Trabajos de campo y fotointerpretación.

SECTION GEOMETRY/GEOMETRIA DE LA SECCION

LENGTH/LONGITUD: $68.2 \mathrm{~km}$ (66.1 km cumulative)

AVERAGE STRIKE/RUMBO PROMEDIO: $11.1^{\circ} \pm 8^{\circ}\left(\mathrm{N} 11.1^{\circ} \mathrm{E} \pm 8^{\circ}\right)$

AVERAGE DIP/INCLINACION PROMEDIO: Vertical

SENSE OF MOVEMENT/SENTIDO DE MOVIMIENTO: Dextral

Comments/Comentarios: This fault section is more than $20 \mathrm{~km}$ long, trending $\mathrm{N} 10^{\circ} \mathrm{E}$. Lateral and vertical displacements yet to be quantified.

Falla de más de $20 \mathrm{~km}$ de largo, de dirección promedio $\mathrm{N} 10^{\circ} \mathrm{E}$. Los desplazamientos laterales y verticales no son todavia cuantificados.

GEOMORPHIC EXPRESSION/EXPRESION GEOMORFOLOGICA: This fault section is located in a very rainy region, impeding a good observation of the fault's morphology. Uplift of the coast along this part of the fault zone was observed after the 1960 earthquake.

Este seccion de la falla se encuentra en una zona muy lluviosa lo que impide una buena observación de la morfología de la falla. Se observó levantamiento de la costa en la zona de la falla posteriormente al sismo de 1960

RECURRENCE INTERVAL/INTERVALO DE RECURRENCIA: Unknown/desconocido

SLIP RATE/TASA DE MOVIMIENTO: Unknown/desconocida

TIME OF MOST RECENT OF MOVEMENT/EDAD DEL ULTIMO MOVIMIENTO: Quaternary/Cuaternario $(<1.6 \mathrm{Ma})$

\section{CH-18C, PUYUHUAPI FAULT, SECTION OF LIQUIÑE-OFQUI FAULT}

SECTION NUMBER/NUMERO DE LA SECCION: CH-18c

SECTION NAME/NOMBRE DE LA SECCION: Puyuhuapi fault (section of Liquiñe-Ofqui fault/seccion de falla Liquiñe-Ofqui)

SYNOPSIS AND GEOLOGIC SETTING/SINOPSIS Y AMBIENTE GEOLOGICO: Quaternary activity. General neotectonic field studies. Main Cordillera. This fault cuts sediment of probable Neogene to Quaternary age. The fault also cuts $1.6 \mathrm{Ma}$ old intrusive rock. This fault is compatible with an NE-SW transpressional tectonic regime.

Actividad cuaternaria. Estudios neotectónicos de campo generales. Cordillera Principal. Corta sedimentos de edad neógena a cuaternaria. Una roca intrusiva de 1.6 Ma está cortada por la falla. Esta falla es compatible con un régimen tectónico en transpresión NE-SW.

COMPILER, AFFILIATION, \& DATE OF COMPILATION/COMPILADOR, AFILIACION Y FECHA DE COMPILACION: Alain Lavenu, IRD, France, and RicardoThiele, Univ. de Chile; Chile, 08-1997.

TYPE OF STUDIES/TIPOS DE ESTUDIOS: Fieldwork and interpretation of aerial photographs.

Trabajos de campo y fotointerpretación. 
SECTION GEOMETRY/GEOMETRIA DE LA SECCION

LENGTH/LONGITUD: 163.4 km (174.7 km cumulative)

AVERAGE STRIKE/RUMBO PROMEDIO: $6.9^{\circ} \pm 14^{\circ}\left(\mathrm{N} 6.9^{\circ} \mathrm{E} \pm 14^{\circ}\right)$

AVERAGE DIP/INCLINACION PROMEDIO: Vertical

SENSE OF MOVEMENT/SENTIDO DE MOVIMIENTO: Dextral

Comments/Comentarios: This fault section is more than $15 \mathrm{~km}$ long, trending $\mathrm{N} 10^{\circ} \mathrm{E}$. Lateral and vertical displacements yet to be quantified.

Este seccion de la falla de más de $15 \mathrm{~km}$ de largo, de dirección promedio $\mathrm{N} 10^{\circ} \mathrm{E}$. Los desplazamientos laterales y verticales no son todavia cuantificados.

GEOMORPHIC EXPRESSION/EXPRESION GEOMORFOLOGICA: This fault section is located in a very rainy region, impeding a good observation of the fault's morphology.

Esta sección de falla se encuentra en una zona muy lluviosa lo que impide una buena observación de la morfología de la falla.

RECURRENCE INTERVAL/INTERVALO DE RECURRENCIA: Unknown/desconocido

SLIP RATE/TASA DE MOVIMIENTO: Unknown/desconocida

TIME OF MOST RECENT OF MOVEMENT/EDAD DEL ULTIMO MOVIMIENTO: Quaternary/Cuaternario $(<1.6 \mathrm{Ma})$

\section{CH-18D, SAN RAFAEL FAULT, SECTION OF LIQUIÑE-OFQUI FAULT}

SECTION NUMBER/NUMERO DE LA SECCION: CH-18d

SECTION NAME/NOMBRE DE LA SECCION: San Rafael fault (section of Liquiñe-Ofqui fault/seccion de falla Liquiñe-Ofqui)

SYNOPSIS AND GEOLOGIC SETTING/SINOPSIS Y AMBIENTE GEOLOGICO: Quaternary activity, described in 1989 (Wood, 1989). General neotectonic field studies. Patagonian Batholith. This fault cuts sediment Neogene to Quaternary in age.

Actividad cuaternaria, descrita en 1989 (Wood, 1989). Estudios neotectónicos de campo generales.

Batólito Patagónico. Corta sedimentos de edad neógena a cuaternaria.

COMPILER, AFFILIATION, \& DATE OF COMPILATION/COMPILADOR, AFILIACION Y FECHA DE

COMPILACION: Alain Lavenu, IRD, France, and RicardoThiele, Univ. de Chile; Chile, 08-1997.

TYPE OF STUDIES/TIPOS DE ESTUDIOS: Fieldwork and interpretation of aerial photographs.

Trabajos de campo y fotointerpretación.

SECTION GEOMETRY/GEOMETRIA DE LA SECCION

LENGTH/LONGITUD: $116.0 \mathrm{~km}$ (111.7 km cumulative)

AVERAGE STRIKE/RUMBO PROMEDIO: $25.2^{\circ} \pm 9^{\circ}\left(\mathrm{N} 25.2^{\circ} \mathrm{E} \pm 9^{\circ}\right)$

AVERAGE DIP/INCLINACION PROMEDIO: Vertical

SENSE OF MOVEMENT/SENTIDO DE MOVIMIENTO: Dextral

Comments/Comentarios: This fault section is more than $25 \mathrm{~km}$ long, trending $\mathrm{N} 10^{\circ} \mathrm{E}$. Lateral and vertical displacements yet to be quantified.

Falla de más de $15 \mathrm{~km}$ de largo, de dirección promedio N10E. Los desplazamientos laterales y

verticales no son todavia cantificados.

GEOMORPHIC EXPRESSION/EXPRESION GEOMORFOLOGICA: Forms $3 \mathrm{~m}$ high scarp. There are sag ponds and swamps along the scarp. Scarp's length exceeds $25 \mathrm{~km}$ on aerial photographs and Landsat images.

Escarpe de $3 \mathrm{~m}$ de alto. Presencia de lagunas y pantanos a lo largo del escarpe. La longitud del escarpe en fotos aereas y imagenes Landsat pasa los $25 \mathrm{~km}$.

RECURRENCE INTERVAL/INTERVALO DE RECURRENCIA: Unknown/desconocido

Comments/Comentarios: Vertical movements produce flooding of woodlands in southern end of block to the west of the fault.

Movimientos verticales provocan anegamiento de bosque en el bloque oeste de la falla en su extremo sur.

SLIP RATE/TASA DE MOVIMIENTO: Unknown/desconocida

TIME OF MOST RECENT OF MOVEMENT/EDAD DEL ULTIMO MOVIMIENTO: Quaternary/Cuaternario $(<1.6 \mathrm{Ma})$ 


\section{REFERENCES/REFERENCIAS}

Bruggen, J., 1950, Fundamento de la Geología de Chile: Santiago, Chile, Instituto Geografico Militar, 374 p.

Cembrano, J., 1998, Kinematics and timing of intra-arc deformation at the southern Andes plate boundary zone: Ph.D. Thesis, University of Dalhousie, $231 \mathrm{pp}$.

Cembrano, J., Hervé, F., Lavenu, A., 1996, The Liquiñe-Ofqui fault zone: a long lived intra-arc fault system in southern Chile: Tectonophysics, v. 259, p. 55-66.

Forsythe, R., Nelson, E., 1985, Geological manifestations of ridge collision: evidence from the Golfo de PenasTaitao basin: Tectonics, v. 4, no. 5, p. 477-495.

Hervé, F., 1994, The southern Andes between $39^{\circ}$ and $44^{\circ} S$ latitude: the geological signature of a transpressure tectonic regime related to a magmatic arc, in K.J. Reutter, E. Scheuber and P.J. Wigger (eds), Tectonics of the Southern central Andes, p. 243-248.

Herve, F., Araya, E., Fuenzalida, J., Solano, A., 1979, Edades radiométricas y tectónica neógena en el sector costero de Chiloé continental, $X^{a}$ Región: Actas $2{ }^{\circ}$ Cong. Geol. Chileno, v. I, p. F1-F18.

Herve, F., Moreno, H., Parada, M.A., 1974, Granitoids of the Andean Range of Valdivia Province, Chile: Pacific Geology, v. 8, p. 39-45.

Hervé, F., Thiele, R., 1987, Estado de conocimiento de las megafallas en Chile y su significado tectónico. Comunicaciones, v. 38, p. 67-91.

Lavenu, A., and Cembrano, J., 1999, Compressional- and transpressional-stress pattern for Pliocene and Quaternary brittle deformation in fore arc and intra-arc zones (Andes of Central and Southern Chile): Journal of Structural Geology, v. 21, p. 1669-1691.

Lavenu, A., Cembrano, J., 1994, Neotectónica de rumbo dextral en la zona de falla Liquiñe-Ofqui-Geometría, cinemática y tensor de esfuerzo: $7^{\circ}$ Cong. Geol. Chileno, v. 1, p. 81-85, Concepción.

Solano, A., 1979 , Geología del sector costero de Chiloé continental entre los $41^{\circ} 50^{\prime}$ y $42^{\circ} 10^{\prime}$ de latitud sur: Santiago, Universidad de Chile, Dpto. de Geología, Memoria de Titulo, 122 pp.

Steifel, J., 1970, Das andesprofil im Bereich des $45^{\circ}$ südlichen Breitengrades: Geol. Rundchau, v. 59, no. 3, p. 961979.

Thiele, R., Herve, F., Parada, M.A., Godoy, E., 1986, La Megafalla Liquiñe-Ofqui en el fiordo de Reloncaví (4130'), Chile: Comunicaciones, v. 37, p. 31-47.

Wood, R.M., 1989, Fallamiento normal reciente en la Laguna de San Rafael, Provincia de Aysen, Chile: Austral. Comunicaciones, Depto. de Geología, Univ. de Chile, v. 40, p. 57-68.

\section{CH-19, RIO SAN JUAN FAULT}

FAULT NUMBER/NUMERO DE LA FALLA: $\mathrm{CH}-19$

FAULT NAME/NOMBRE DE LA FALLA: Rio San Juan

SYNOPSIS AND GEOLOGIC SETTING/SINOPSIS Y AMBIENTE GEOLOGICO: Quaternary activity.

According to Cunningham (1993), the fault is $30 \mathrm{~km}$ long. Patagonian Orocline.

Actividad cuaternaria. Según Cunningham (1993) la falla tiene una longitud de $30 \mathrm{~km}$. Oroclino

Patogoniano.

COMPILER, AFFILIATION, \& DATE OF COMPILATION/COMPILADOR, AFILIACION Y FECHA DE

COMPILACION: Alain Lavenu, IRD, France, and RicardoThiele, Univ. de Chile; Chile, 08-1997.

TYPE OF STUDIES/TIPOS DE ESTUDIOS: Fieldwork and interpretation of aerial photographs.

Trabajos de campo y fotointerpretación.

FAULT GEOMETRY/GEOMETRIA DE LA FALLA

LENGTH/LONGITUD: $33.0 \mathrm{~km}$ (33.1 km cumulative)

AVERAGE STRIKE/RUMBO PROMEDIO: $39.6^{\circ} \pm 6^{\circ}\left(\mathrm{N} 39.6^{\circ} \mathrm{E} \pm 6^{\circ}\right)$

AVERAGE DIP/INCLINACION PROMEDIO: Vertical

SENSE OF MOVEMENT/SENTIDO DE MOVIMIENTO: Sinistral

Comments/Comentarios: This fault section is more than $30 \mathrm{~km}$ long, with an average NW-SE direction.

Este seccion de la falla de más de $30 \mathrm{~km}$ de largo, de dirección promedio NW-SE.

GEOMORPHIC EXPRESSION/EXPRESION GEOMORFOLOGICA: Forms linear truncation of river

meanders.

Truncamiento lineal de meandros de ríos.

RECURRENCE INTERVAL/INTERVALO DE RECURRENCIA: Unknown/desconocido

SLIP RATE/TASA DE MOVIMIENTO: Unknown/desconocida 
TIME OF MOST RECENT OF MOVEMENT/EDAD DEL ULTIMO MOVIMIENTO: Quaternary/Cuaternario $(<1.6 \mathrm{Ma})$

REFERENCES/REFERENCIAS

Cunningham, W., 1993, Strike-slip faults in the Southernmost Andes and the development of the Patagonian Orocline: Tectonics, v. 12, no. 1, p. 169-186.

Katz, H., 1964, Some new concepts on geosynclinal development and mountain building at the southern end of South America: Proceeding of the 22nd. International Geological Congress, New Dehli, v. 4, p. 241-255.

\section{CH-20, LAGO FAGNANO FAULT}

FAULT NUMBER/NUMERO DE LA FALLA: CH-20

Comments/Comentarios: In Argentina, this is also the Lago Fagnano fault [AR-56]. En Argentina, este es la falla Lago Fagnano [AR-56].

FAULT NAME/NOMBRE DE LA FALLA: Lago Fagnano

SYNOPSIS AND GEOLOGIC SETTING/SINOPSIS Y AMBIENTE GEOLOGICO: Quaternary activity.

Klepeis (1994), deduced this activity from seismic activity in the southern tip of South America.

Cunningham (1993) indicates a left-lateral displacement for this fault.

Actividad cuaternaria. Para Klepeis esta actividad se deduce de la actividad sismica en el extremo sur de

America del sur (Klepeis, 1994). Para Cunningham (1993) esta falla presenta un desplazamiento senestral.

COMPILER, AFFILIATION, \& DATE OF COMPILATION/COMPILADOR, AFILIACION Y FECHA DE

COMPILACION: Alain Lavenu, IRD, France, and RicardoThiele, Univ. de Chile; Chile, 08-1997.

TYPE OF STUDIES/TIPOS DE ESTUDIOS: Fieldwork/Trabajos de campo.

FAULT GEOMETRY/GEOMETRIA DE LA FALLA:

LENGTH/LONGITUD: $377.5 \mathrm{~km}$ (392.4 km cumulative)

Comments/Comentarios: Does not include $96 \mathrm{~km}$ of fault AR-56 in Argentina/No incluir $96 \mathrm{~km}$ de falla

AR-56 en Argentina.

AVERAGE STRIKE/RUMBO PROMEDIO: $-57.3^{\circ} \pm 7^{\circ}\left(\mathrm{N} 57.3^{\circ} \mathrm{W} \pm 7^{\circ}\right)$

Comments/Comentarios: Does not include fault AR-56 in Argentina/No incluir falla AR-56 en Argentina.

AVERAGE DIP/INCLINACION PROMEDIO: Subvertical

SENSE OF MOVEMENT/SENTIDO DE MOVIMIENTO: Sinistral and sinistral-normal

NUMBER OF SECTIONS/NOMBRE DE LA SECCIONES: 2 [CH-20a, CH-20b],

Comments/Comentarios: For this compilation, two sections were defined for the Lago Fagnano fault in

Chile.

Para esta compilación, dos secciones fueron definidas para la Falla Fagnano en Chile.

\section{CH-20A, WESTERN SECTION OF LAGO FAGNANO FAULT}

\section{SECTION NUMBER/NUMERO DE LA SECCION: CH-20a}

SECTION NAME/NOMBRE DE LA SECCION: Western section of Lago Fagnano fault/seccion occidental de falla Lago Fagnano.

SYNOPSIS AND GEOLOGIC SETTING/SINOPSIS Y AMBIENTE GEOLOGICO: Quaternary activity. Klepeis (1994), deduced this activity from seismic activity in the southern tip of South America.

Cunningham (1993) indicates a left-lateral displacement for this fault.

Actividad cuaternaria. Para Klepeis esta actividad se deduce de la actividad sismica en el extremo sur de America del sur (Klepeis, 1994). Para Cunningham (1993) esta falla presenta un desplazamiento sinestral.

COMPILER, AFFILIATION, \& DATE OF COMPILATION/COMPILADOR, AFILIACION Y FECHA DE

COMPILACION: Alain Lavenu, IRD, France, and RicardoThiele, Univ. de Chile; Chile, 08-1997.

TYPE OF STUDIES/TIPOS DE ESTUDIOS: Interpretation of aerial photographs/Fotointerpretación. 
SECTION GEOMETRY/GEOMETRIA DE LA SECCION

LENGTH/LONGITUD: $282.3 \mathrm{~km}$ (280.1 km cumulative)

AVERAGE STRIKE/RUMBO PROMEDIO: $-55.6^{\circ} \pm 5^{\circ}\left(\mathrm{N} 55.6^{\circ} \mathrm{W} \pm 5^{\circ}\right)$

AVERAGE DIP/INCLINACION PROMEDIO: Vertical

SENSE OF MOVEMENT/SENTIDO DE MOVIMIENTO: Sinistral and sinistral-normal

Comments/Comentarios: The Chilean part of the fault is more than $20 \mathrm{~km}$ long, trending $\mathrm{N} 100^{\circ} \mathrm{E}$.

La parte chilena de la falla mide más de $20 \mathrm{~km}$, con una dirección promedio $\mathrm{N} 100^{\circ} \mathrm{E}$.

GEOMORPHIC EXPRESSION/EXPRESION GEOMORFOLOGICA: According to Cunningham (1993), the

fault displaces lithological contacts, cuts meanders of the Rio Cortados, and displaces glacial forms, as

well as forms landslides and scarps.

Según Cunningham (1993) se nota la presencia de contactos litológicos desplazados, deslizamientos

de terreno, escarpes, meandros de Rio Cortados, formas glaciales desplazadas.

RECURRENCE INTERVAL/INTERVALO DE RECURRENCIA: Unknown/desconocido

SLIP RATE/TASA DE MOVIMIENTO: Unknown/desconocida

TIME OF MOST RECENT OF MOVEMENT/EDAD DEL ULTIMO MOVIMIENTO: Quaternary/Cuaternario $(<1.6 \mathrm{Ma})$

Comments/Comentarios: The activity could be more recent, possibly post glacial ( $<15 \mathrm{ka})$ based on

Cunningham's observations.

El movimiento podría ser más reciente, de edad post-glacial ( $<15 \mathrm{ka})$ según observaciones de

Cunningham (1993).

\section{CH-20B, EASTERN SECTION OF LAGO FAGNANO FAULT}

SECTION NUMBER/NUMERO DE LA SECCION: CH-20b

SECTION NAME/NOMBRE DE LA SECCION: Eastern section of Lago Fagnano fault/seccion oriental de falla Lago Fagnano.

Comments/Comentarios: In Argentina, this is the Lago Fagnano fault [AR-56]. En Argentina, este es la falla Lago Fagnano [AR-56].

SYNOPSIS AND GEOLOGIC SETTING/SINOPSIS Y AMBIENTE GEOLOGICO: Quaternary activity.

Klepeis (1994), deduced this activity from seismic activity in the southern tip of South America.

Cunningham (1993) indicates a left-lateral displacement for this fault.

Actividad cuaternaria. Para Klepeis esta actividad se deduce de la actividad sismica en el extremo sur de America del sur (Klepeis, 1994). Para Cunningham (1993) esta falla presenta un desplazamiento sinestral.

COMPILER, AFFILIATION, \& DATE OF COMPILATION/COMPILADOR, AFILIACION Y FECHA DE COMPILACION: Alain Lavenu, IRD, France, and RicardoThiele, Univ. de Chile; Chile, 08-1997.

TYPE OF STUDIES/TIPOS DE ESTUDIOS: Fieldwork and interpretation of aerial photographs.

Trabajos de campo y fotointerpretación.

SECTION GEOMETRY/GEOMETRIA DE LA SECCION

LENGTH/LONGITUD: $112.3 \mathrm{~km}$ (112.4 km cumulative)

AVERAGE STRIKE/RUMBO PROMEDIO: $-61.5^{\circ} \pm 6^{\circ}\left(\mathrm{N} 61.5^{\circ} \mathrm{W} \pm 6^{\circ}\right)$

Comments/Comentarios: The Chilean part of the fault trends $\mathrm{N} 100^{\circ} \mathrm{E} . / \mathrm{L}$ a parte chilena es una dirección promedio $\mathrm{N} 100^{\circ} \mathrm{E}$.

AVERAGE DIP/INCLINACION PROMEDIO: Vertical

SENSE OF MOVEMENT/SENTIDO DE MOVIMIENTO: Sinistral and sinistral-normal

Comments/Comentarios: The Chilean part of the fault is more than $20 \mathrm{~km}$ long and trends $\mathrm{N} 100^{\circ} \mathrm{E}$.

La parte chilena de la falla mide más de $20 \mathrm{~km}$, con una dirección promedio $\mathrm{N} 100^{\circ} \mathrm{E}$.

GEOMORPHIC EXPRESSION/EXPRESION GEOMORFOLOGICA: West part of fault is under water.

La falla al oeste es submarine.

RECURRENCE INTERVAL/INTERVALO DE RECURRENCIA: Unknown/desconocido

SLIP RATE/TASA DE MOVIMIENTO: Unknown/desconocida 
TIME OF MOST RECENT OF MOVEMENT/EDAD DEL ULTIMO MOVIMIENTO: Quaternary/Cuaternario $(<1.6 \mathrm{Ma})$

Comments/Comentarios: The activity could be more recent, possibly post glacial ( $<15 \mathrm{ka})$ based on Cunningham's observations.

El movimiento podría ser más reciente, de edad post-glacial (<15 ka) según observaciones de Cunningham (1993).

REFERENCES/REFERENCIAS

Cunningham, W., 1993, Strike-slip faults in the Southernmost Andes and the development of the Patagonian orocline: Tectonics, v. 12, no. 1, p. 169-186.

Katz, H., 1964, Some new concepts on geosynclinal development and mountain building at the southern end of South America: Proceeding of the 22nd. International Geological Congress, New Dehli, v. 4, p. 241-255.

Klepeis, K.A., 1994, The Magallanes and Deseado fault zones: Major segments of the South American-Scotia transform plate boundary in southermost South America, Tierra de Fuego: Journal of Geophysical Research, v. 99, no. B11, p. 22001-22014. 


\section{TABLE 2. QUATERNARY FAULTS OF CHILE (FALLAS CUATERNARIAS DE CHILE)}

\begin{tabular}{|c|c|c|c|c|}
\hline $\begin{array}{l}\text { Number } \\
\text { Número }\end{array}$ & $\begin{array}{l}\text { Name of structure } \\
\text { Nombre de estructura }\end{array}$ & $\begin{array}{l}\text { Sense of } \\
\text { movement } \\
\text { Sentido de } \\
\text { movimiento }\end{array}$ & $\begin{array}{l}\text { Time of most } \\
\text { recent faulting } \\
\text { Edad del último } \\
\text { movimiento }\end{array}$ & $\begin{array}{l}\text { Slip rate }(\mathrm{mm} / \mathrm{yr}) / \\
\text { Tasa de movimiento } \\
\text { (mm/año) }\end{array}$ \\
\hline $\mathrm{CH}-01$ & Atacama fault (falla de Atacama) & & & \\
\hline $\mathrm{CH}-01 \mathrm{a}$ & $\begin{array}{l}\text { Salar Grande fault (falla del Salar } \\
\text { Grande ) }\end{array}$ & Dextral-reverse & $<1.6 \mathrm{Ma}$ & $\begin{array}{l}\text { Unknown/ } \\
\text { desconocida }\end{array}$ \\
\hline $\mathrm{CH}-01 \mathrm{~b}$ & $\begin{array}{l}\text { Punta de Lobos fault (falla de Punta } \\
\text { de Lobos) }\end{array}$ & Dextral-reverse & $<1.6 \mathrm{Ma}$ & $\begin{array}{l}\text { Unknown/ } \\
\text { desconocida }\end{array}$ \\
\hline $\mathrm{CH}-01 \mathrm{c}$ & $\begin{array}{l}\text { Fault north of Carmen Salar (falla } \\
\text { norte del Salar del Carmen) }\end{array}$ & Dextral-reverse & $<1.6 \mathrm{Ma}$ & $\begin{array}{l}\text { Unknown/ } \\
\text { desconocida }\end{array}$ \\
\hline $\mathrm{CH}-01 \mathrm{~d}$ & $\begin{array}{l}\text { Fault south of Carmen Salar (falla sur } \\
\text { del Salar del Carmen) }\end{array}$ & Normal & Historic (1995) & $\begin{array}{l}\text { Unknown/ } \\
\text { desconocida }\end{array}$ \\
\hline $\mathrm{CH}-01 \mathrm{e}$ & $\begin{array}{l}\text { Sierra de Remiendos fault (falla de } \\
\text { Sierra de Remiendos) }\end{array}$ & Normal & Historic (1995) & $\begin{array}{l}\text { Unknown/ } \\
\text { desconocida }\end{array}$ \\
\hline $\mathrm{CH}-02$ & $\begin{array}{l}\text { Morro Mejillones fault (falla del Morro } \\
\text { Mejillones) }\end{array}$ & Normal & $<1.6 \mathrm{Ma}$ & $\begin{array}{l}\text { Unknown/ } \\
\text { desconocida }\end{array}$ \\
\hline $\mathrm{CH}-03$ & $\begin{array}{l}\text { Cerro Gordo fault, Atacama fault zone } \\
\text { (falla del Cerro Gordo, sistema falla de } \\
\text { Atacama) }\end{array}$ & Normal-sinistral & $\begin{array}{l}<1.6 \mathrm{Ma} \\
\quad(<15 \mathrm{ka} ?)\end{array}$ & $\begin{array}{l}\text { Unknown/ } \\
\text { desconocida }\end{array}$ \\
\hline $\mathrm{CH}-04$ & $\begin{array}{l}\text { Cerro Moreno fault, Atacama fault zone } \\
\text { (falla del Cerro Moreno, sistema falla } \\
\text { de Atacama) }\end{array}$ & Normal-sinistral & $\begin{array}{l}<1.6 \mathrm{Ma} \\
\quad(<15 \mathrm{ka} ?)\end{array}$ & $\begin{array}{l}\text { Unknown/ } \\
\text { desconocida }\end{array}$ \\
\hline $\mathrm{CH}-05$ & $\begin{array}{l}\text { Cerro Fortuna fault, Atacama fault zone } \\
\text { (falla Cerro Fortuna, sistema falla de } \\
\text { Atacama) }\end{array}$ & Normal & $<1.6 \mathrm{Ma}$ & $\begin{array}{l}\text { Unknown/ } \\
\text { desconocida }\end{array}$ \\
\hline $\mathrm{CH}-06$ & $\begin{array}{l}\text { Western Back Thrust of Cerro de } \\
\text { Purilactis (Falla Western Back Thrust } \\
\text { del Cerro de Purilactis }\end{array}$ & Reverse & $<1.6 \mathrm{Ma}$ & $\begin{array}{l}\text { Unknown/ } \\
\text { desconocida }\end{array}$ \\
\hline $\mathrm{CH}-07$ & $\begin{array}{l}\text { Frontal Domeyko Thrust of Llano de la } \\
\text { Paciencia (Falla Frontal Domeyko } \\
\text { Thrust del Llano de la Paciencia }\end{array}$ & Reverse & $<1.6 \mathrm{Ma}$ & $\begin{array}{l}\text { Unknown/ } \\
\text { desconocida }\end{array}$ \\
\hline $\mathrm{CH}-08$ & $\begin{array}{l}\text { Ignimbrite Back Thrust of Llano de la } \\
\text { Paciencia (Falla Ignimbrite Back } \\
\text { Thrust del Llano de la Paciencia }\end{array}$ & Reverse & $<1.6 \mathrm{Ma}$ & $\begin{array}{l}\text { Unknown/ } \\
\text { desconocida }\end{array}$ \\
\hline $\mathrm{CH}-09$ & $\begin{array}{l}\text { Eastern Frontal Thrust of Llano de la } \\
\text { Paciencia (Falla Eastern Frontal } \\
\text { Thrust del Llano de la Paciencia }\end{array}$ & Reverse & $<1.6 \mathrm{Ma}$ & $\begin{array}{l}\text { Unknown/ } \\
\text { desconocida }\end{array}$ \\
\hline $\mathrm{CH}-10$ & $\begin{array}{l}\text { Cordillera of the Sal Frontal Thrust fault } \\
\text { (Falla Frontal Thrust de la Cordillera de } \\
\text { la Sal) }\end{array}$ & Reverse & $<1.6 \mathrm{Ma}$ & $\begin{array}{l}\text { Unknown/ } \\
\text { desconocida }\end{array}$ \\
\hline $\mathrm{CH}-11$ & Tucúcaro fault (falla Tucúcaro) & Reverse & $<1.6 \mathrm{Ma}$ & $\begin{array}{l}\text { Unknown/ } \\
\text { desconocida }\end{array}$ \\
\hline $\mathrm{CH}-12$ & $\begin{array}{l}\text { Faults south of Caldera (fallas al sur de } \\
\text { Caldera) }\end{array}$ & Normal & $\begin{array}{l}<1.6 \mathrm{Ma} \\
\quad(<125 \mathrm{ka})\end{array}$ & 0.30 \\
\hline
\end{tabular}


TABLE 2-CONTINUED

\begin{tabular}{|c|c|c|c|c|}
\hline $\begin{array}{l}\text { Number } \\
\text { Número }\end{array}$ & $\begin{array}{l}\text { Name of structure } \\
\text { Nombre de estructura }\end{array}$ & $\begin{array}{l}\text { Sense of } \\
\text { movement } \\
\text { Sentido de } \\
\text { movimiento }\end{array}$ & $\begin{array}{l}\text { Time of most } \\
\text { recent faulting } \\
\text { Edad del último } \\
\text { movimiento }\end{array}$ & $\begin{array}{l}\text { Slip rate }(\mathrm{mm} / \mathrm{yr}) / \\
\text { Tasa de movimiento } \\
\text { (mm/año) }\end{array}$ \\
\hline $\mathrm{CH}-13$ & $\begin{array}{l}\text { Faults East of Bahía Inglesa (fallas este } \\
\text { de Bahía Inglesa) }\end{array}$ & Normal & $\begin{array}{l}<1.6 \mathrm{Ma} \\
\quad(<125 \mathrm{ka})\end{array}$ & 0.27 \\
\hline $\mathrm{CH}-14$ & $\begin{array}{l}\text { Faults of Alto del Fraile (fallas de Alto del } \\
\text { Fraile) }\end{array}$ & Normal & $<1.6 \mathrm{Ma}$ & $\begin{array}{l}\text { Unknown/ } \\
\text { desconocida }\end{array}$ \\
\hline $\mathrm{CH}-15$ & $\begin{array}{l}\text { Fault of San Jose de Maipo (falla de San } \\
\text { José de Maipo) }\end{array}$ & Reverse & $<1.6 \mathrm{Ma}$ & $\begin{array}{l}\text { Unknown/ } \\
\text { desconocida }\end{array}$ \\
\hline $\mathrm{CH}-16$ & Fault of Esperanza (falla de Esperanza) & Reverse & $<1.6 \mathrm{Ma}$ & $\begin{array}{l}\text { Unknown/ } \\
\text { desconocida }\end{array}$ \\
\hline $\mathrm{CH}-17$ & Fault of Victoria (falla de Victoria) & Reverse & $<1.6 \mathrm{Ma}$ & $\begin{array}{l}\text { Unknown/ } \\
\text { desconocida }\end{array}$ \\
\hline $\mathrm{CH}-18$ & $\begin{array}{l}\text { Liquiñe-Ofqui fault (falla de Liquiñe- } \\
\text { Ofqui) }\end{array}$ & & & \\
\hline $\mathrm{CH}-18 \mathrm{a}$ & Reloncaví fault (falla de Reloncaví) & Dextral & $<1.6 \mathrm{Ma}$ & $\begin{array}{l}\text { Unknown/ } \\
\text { desconocida }\end{array}$ \\
\hline $\mathrm{CH}-18 \mathrm{~b}$ & Hornopirén fault (falla de Hornopirén) & Dextral & $<1.6 \mathrm{Ma}$ & $\begin{array}{l}\text { Unknown/ } \\
\text { desconocida }\end{array}$ \\
\hline $\mathrm{CH}-18 \mathrm{C}$ & Puyuhuapi fault (falla de Puyuhuapi) & Dextral & $<1.6 \mathrm{Ma}$ & $\begin{array}{l}\text { Unknown/ } \\
\text { desconocida }\end{array}$ \\
\hline $\mathrm{CH}-18 \mathrm{~d}$ & San Rafael fault (falla de San Rafael) & Dextral & $<1.6 \mathrm{Ma}$ & $\begin{array}{l}\text { Unknown/ } \\
\text { desconocida }\end{array}$ \\
\hline $\mathrm{CH}-19$ & Rio San Juan fault (falla Rio San Juan) & Sinistral & $<1.6 \mathrm{Ma}$ & $\begin{array}{l}\text { Unknown/ } \\
\text { desconocida }\end{array}$ \\
\hline $\mathrm{CH}-20$ & Lago Fagnano fault (falla Lago Fagnano) & & & \\
\hline $\mathrm{CH}-20 \mathrm{a}$ & Western section (seccion occidental) & $\begin{array}{l}\text { Sinistral and } \\
\text { sinistral-normal }\end{array}$ & $\begin{array}{l}<1.6 \mathrm{Ma} \\
\quad(<15 \mathrm{ka} ?)\end{array}$ & $\begin{array}{l}\text { Unknown/ } \\
\text { desconocida }\end{array}$ \\
\hline $\mathrm{CH}-20 \mathrm{~b}$ & Eastern section (seccion oriental) & $\begin{array}{l}\text { Sinistral and } \\
\text { sinistral-normal }\end{array}$ & $\begin{array}{l}<1.6 \mathrm{Ma} \\
\quad(<15 \mathrm{ka} ?)\end{array}$ & $\begin{array}{l}\text { Unknown/ } \\
\text { desconocida }\end{array}$ \\
\hline
\end{tabular}

